\title{
Ergot alkaloid biosynthesis in Aspergillus fumigatus : Association with sporulation and clustered genes common among ergot fungi
}

Christine M. Coyle

West Virginia University

Follow this and additional works at: https://researchrepository.wvu.edu/etd

\section{Recommended Citation}

Coyle, Christine M., "Ergot alkaloid biosynthesis in Aspergillus fumigatus : Association with sporulation and clustered genes common among ergot fungi" (2009). Graduate Theses, Dissertations, and Problem Reports. 4453.

https://researchrepository.wvu.edu/etd/4453

This Dissertation is protected by copyright and/or related rights. It has been brought to you by the The Research Repository @ WVU with permission from the rights-holder(s). You are free to use this Dissertation in any way that is permitted by the copyright and related rights legislation that applies to your use. For other uses you must obtain permission from the rights-holder(s) directly, unless additional rights are indicated by a Creative Commons license in the record and/ or on the work itself. This Dissertation has been accepted for inclusion in WVU Graduate Theses, Dissertations, and Problem Reports collection by an authorized administrator of The Research Repository @ WVU.

For more information, please contact researchrepository@mail.wvu.edu. 


\title{
Ergot alkaloid biosynthesis in Aspergillus fumigatus: Association with sporulation and clustered genes common among ergot fungi
}

\author{
Christine M. Coyle \\ Dissertation submitted to the \\ Davis College of Agriculture, Forestry, and Consumer Sciences \\ at West Virginia University \\ in partial fulfillment of the requirements \\ for the degree of \\ Doctor of Philosophy \\ in \\ Genetics and Developmental Biology \\ Daniel G. Panaccione, Ph.D., Chair \\ Kenneth P. Blemings, Ph.D. \\ Joseph B. Morton, Ph.D. \\ Alan J. Sexstone, Ph.D. \\ Sharon L. Wenger, Ph.D. \\ Division of Plant and Soil Sciences \\ Morgantown, West Virginia \\ 2009
}

Keywords: Aspergillus fumigatus, ergot alkaloid biosynthesis, sporulation

Copyright 2009 Christine M. Coyle 


\title{
ABSTRACT \\ Ergot alkaloid biosynthesis in Aspergillus fumigatus: Association with sporulation and clustered genes common among ergot fungi
}

\author{
Christine M. Coyle
}

Ergot alkaloids, indole-derived mycotoxins, interact with multiple monoamine neurotransmitter receptors and cause disease in exposed individuals. They have been well studied in the ergot fungus, Claviceps purpurea, and have been reported in some closely related grass endophytes, as well as the distantly related opportunistic human pathogen Aspergillus fumigatus. A. fumigatus, which sporulates prolifically, produces clavines, specifically festuclavine and fumigaclavines $\mathrm{A}, \mathrm{B}$, and $\mathrm{C}$ in association with asexual spores and the total mass of alkaloids constitutes over $1 \%$ of the spore mass. These alkaloids differ from those of most clavicipitaceous fungi, which consist of different clavines and often more complex lysergic acid derivatives. However, the ergot alkaloid pathways of $A$. fumigatus and ergot fungi are hypothesized to share early biosynthetic steps, diverging at some point after the formation of the intermediate chanoclavine. A homologue of $d m a W$, a gene encoding dimethylallyltryptophan synthase in Neotyphodium endophytes, was found in the A. fumigatus genome. By gene knockout analysis, $d m a W$ was shown to be required for ergot alkaloid production in $A$. fumigatus. Comparison of genes clustered around A. fumigatus dmaW to those clustered with $d m a W$ in the ergot fungi revealed potential homologues that could encode proteins controlling early, shared steps in the pathway. Functional analysis via gene knockout of three additional $A$. fumigatus genes (eas $A$, eas $E$, and $e a s F$ ) rendered mutants with altered alkaloid profiles, demonstrating their involvement in ergot alkaloid biosynthesis. All mutants lacked normal ergot alkaloid production from the latter part of the pathway; complementation with a functional copy of the respective gene, restored normal ergot alkaloid production in each mutant. Analyses of intermediates positioned the products of $e a s F$ and eas $E$ as the second and third enzymatic steps of the pathway. Knockout of eas $A$ caused accumulation of multiple intermediates, including chanoclavine; complementation with the $C$. purpurea eas $A$ gene resulted in accumulation of agroclavine, setoclavine, and its isomer isosetoclavine. These data confirm eas $A$ involvement post chanoclavine synthesis and more specifically assign its role to reduction of chanoclavine aldehyde, the branch point of the two lineage-specific pathways. These mutants, due to their differing ergot alkaloid profiles, are valuable for testing the role of specific ergot alkaloids in animal pathogenesis and toxicoses.

Elucidation of ergot alkaloid biosynthesis, along with the capacity to control the spectrum of alkaloids produced, may be beneficial to agriculture and medicine. Additional studies demonstrated that production of ergot alkaloids was restricted to conidiating cultures. Disruption of $\operatorname{brl} A$, a regulatory gene involved in conidiation, interfered with conidiophore development, as well as ergot alkaloid production. The association of these toxins with sporulation may offer insight into their ecological significance and utility to the fungus. 


\section{DEDICATION}

I would like to dedicate this research to my advisor, Dr. Dan Panaccione. His vision and guidance has made this research possible. He has approached the project with overwhelming insight and enthusiasm, providing encouragement and enlightenment along the way. I appreciate the time and energy he has devoted to teaching and mentoring me as a graduate student. I am extremely grateful for his support, patience, and understanding during my years here at WVU, which made it possible to pursue this degree while balancing life as a mother and wife. It truly has been a privilege to work with Dan. He has helped lay my foundation for success and for this I will be forever thankful. 


\section{ACKNOWLEDGEMENTS}

It is through the support of others that we can accomplish great things. First, I would like to thank my advisor, Dr. Dan Panaccione, and my committee members, Dr. Sharon Wenger, Dr. Ken Blemings, Dr. Joe Morton, and Dr. Alan Sexstone for their assistance and guidance throughout my Ph.D. pursuit. Dr. Dale Karlson, Dr. Jed Doelling, Dr. Joginder Nath, and Dr. Barton Baker have also provided assistance during my time at WVU, along with the Plant and Soil Science administrative assistants. Many thanks to Sherie Edenborn, Kerry Goetz, Shanthi Mulinti, Dawn Chamberlain, Mike Hamstead, and Brian Lewis for their laboratory assistance.

I truly cherish the moments I have spent with my dear colleagues and friends within Environmental Microbiology and Plant Pathology. I am grateful for their support, advice, and camaraderie. I would especially like to recognize Bill Wheeler, Mark Double, Dr. Gary Bissonnette, Sherie Edenborn, Kerry Goetz, Shanthi Mulinti, Dawn Chamberlain, Tiara King, Emily Markle, Zola Msiska, Kelly Mayhew, Brian Lewis, Shawn Kenaley, and Bill Rittenour. In addition, I will never forget the lunch bunch, (This includes you, Dr. Gallegly) for the exhilarating and often enlightening conversations.

I have been blessed with the constant love and support of my family and friends. My parents have provided me with endless guidance and encouragement. My siblings have enriched my life through their friendship. I feel fortunate for the encouragement and support of my in-laws over the last several years. Their willingness to care for my daughter, Grace, has made this experience easier. Grace has been a blessing throughout this endeavor. Her sweet and loving nature is a wonderful welcome, especially after a long day. And last, but definitely not least, my husband Andrew. I am so grateful for his support, love, and encouragement, especially this last year. Keeping life together, especially in the last few months, has been a challenge and his support has made it possible.

I have had a wonderful experience at WVU and will cherish these memories always! 


\section{Table of Contents}

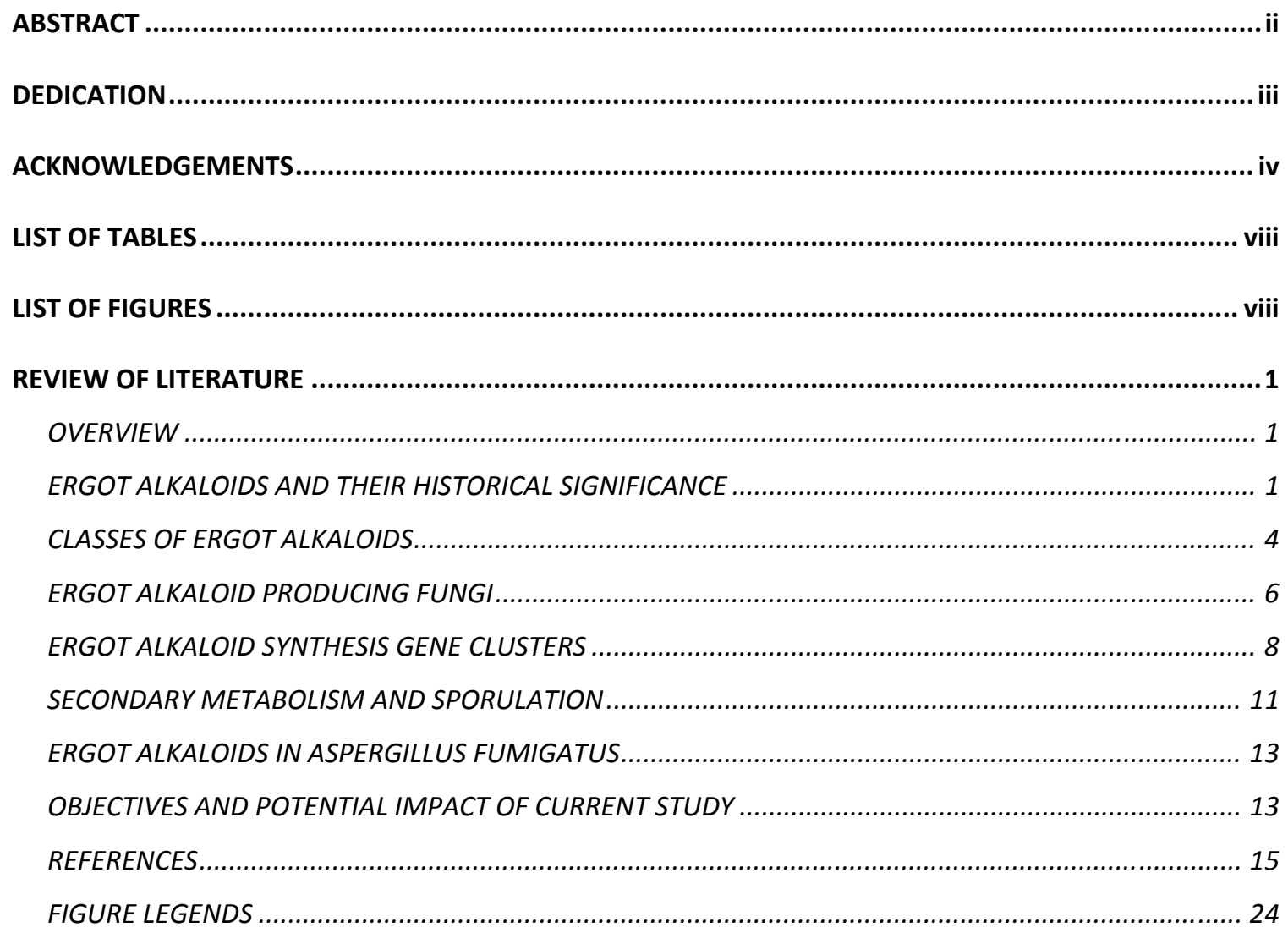

CHAPTER 1: An ergot alkaloid biosynthesis gene and clustered hypothetical genes from Aspergillus

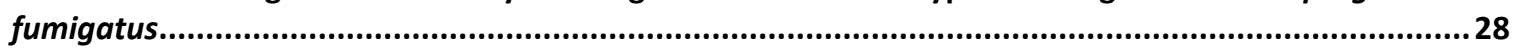

ABSTRACT

CHAPTER 2: Clustered genes, easE and easF, common to Aspergillus fumigatus and ergot fungi control

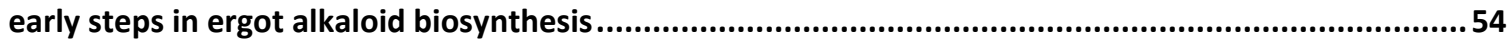

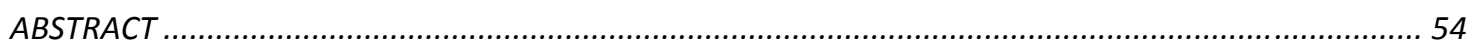

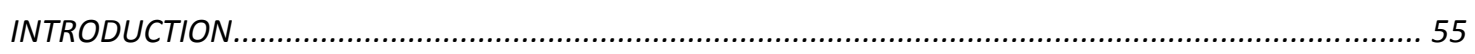

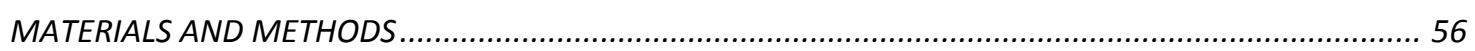

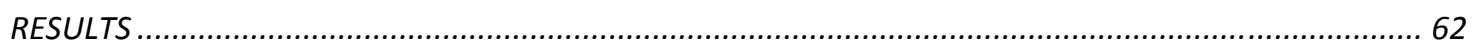




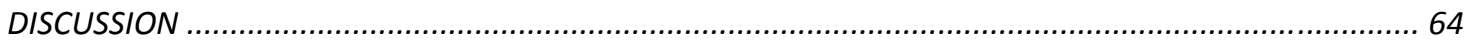

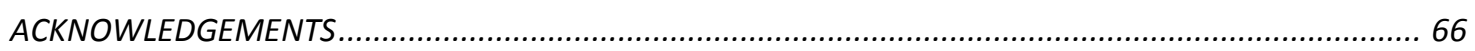

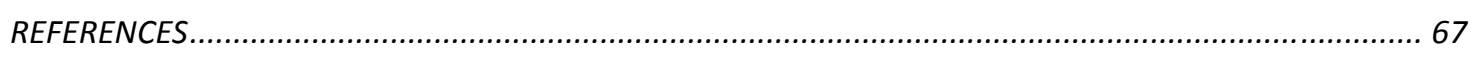

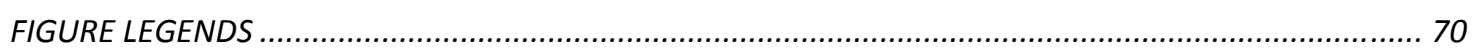

CHAPTER 3: An old yellow enzyme gene is required for ergot alkaloid biosynthesis and controls the branch point between Aspergillus fumigatus and Claviceps purpurea ergot alkaloid pathways ............ 78

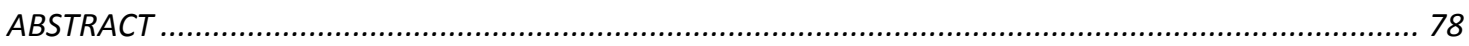

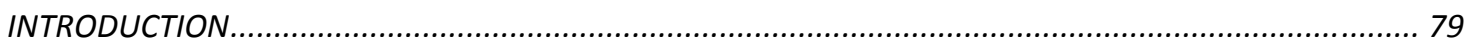

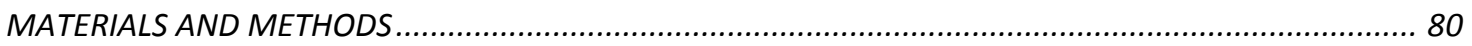

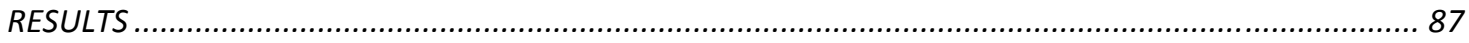

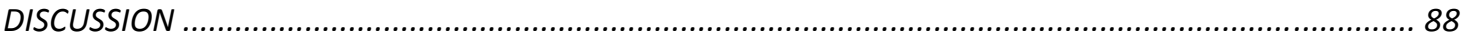

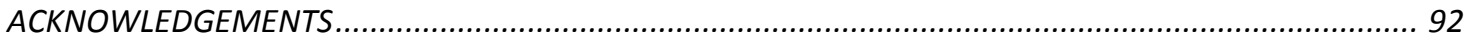

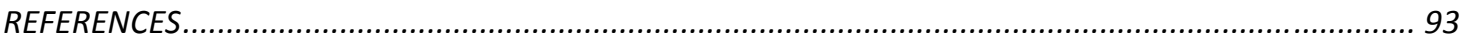

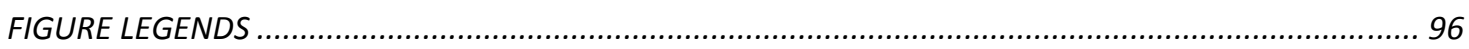

CHAPTER 4: Association of ergot alkaloids with conidiation in Aspergillus fumigatus ...................... 109

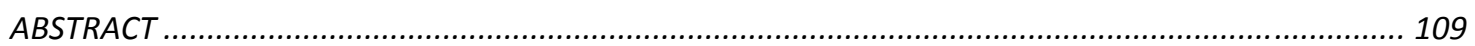

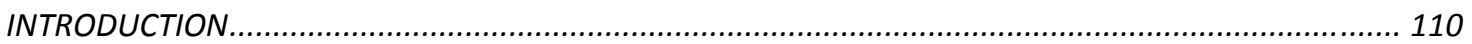

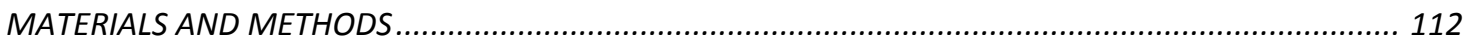

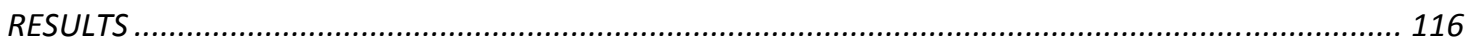

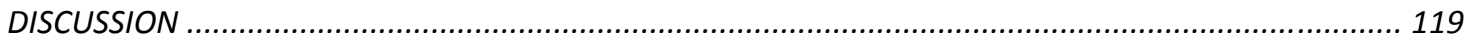

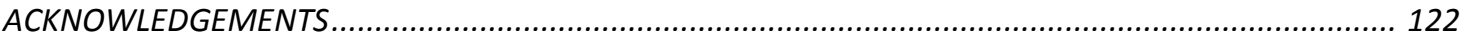

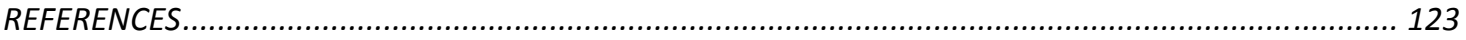

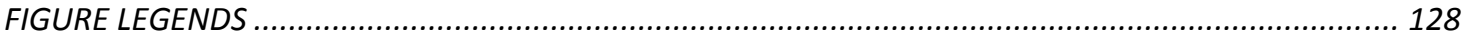

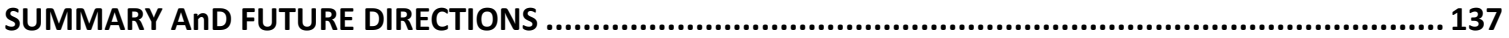

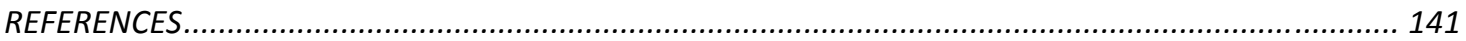

APPENDIX 1: Abundant respirable ergot alkaloids from the common airborne fungus Aspergillus fumigatus.............................................................................................................. 142

ABSTRACT 
APPENDIX 2: Analysis of ergot alkaloids in spore only samples versus entire fungal (plate) cultures in the fungus, Aspergillus fumigatus.............................................................................................. 166

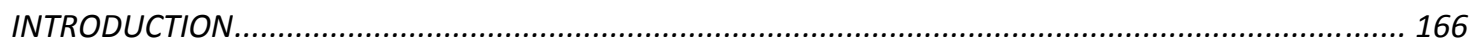

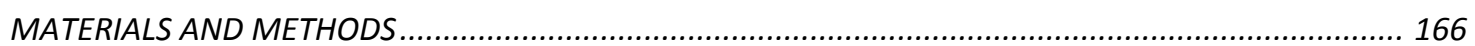

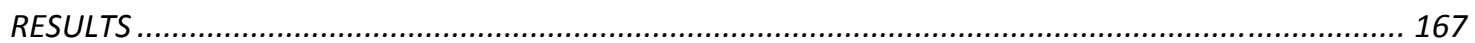

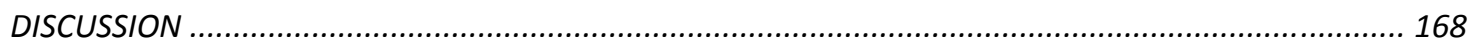

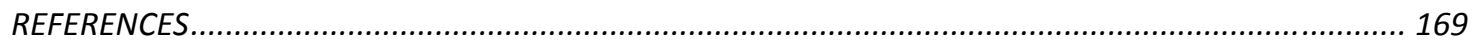

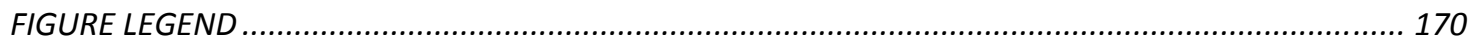

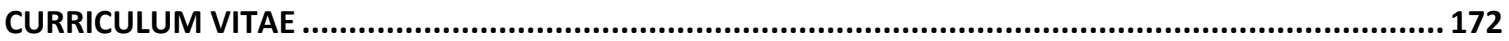




\section{LIST OF TABLES}

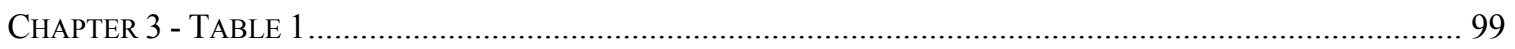

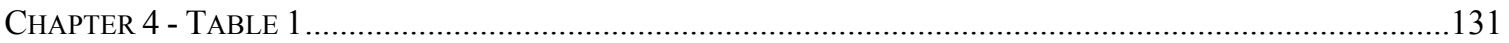

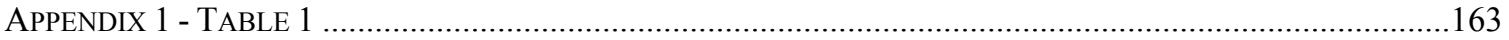

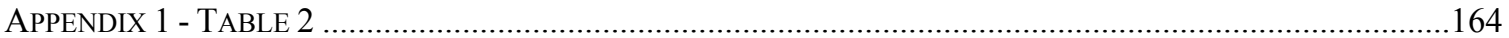

\section{LIST OF FIGURES}

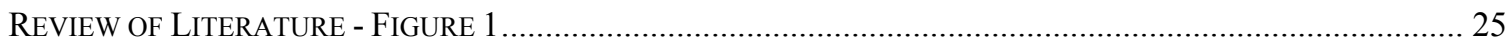

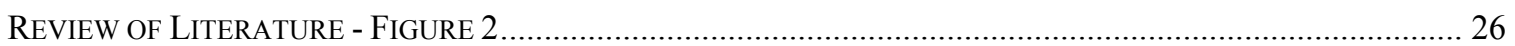

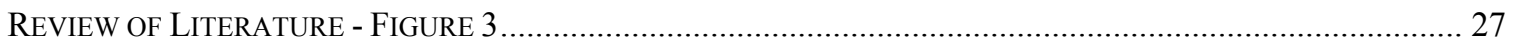

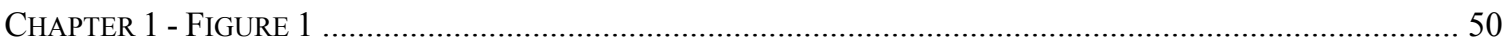

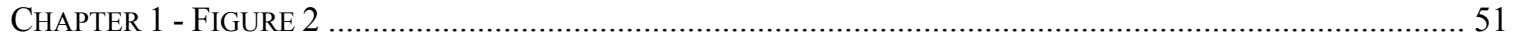

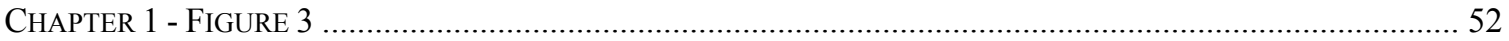

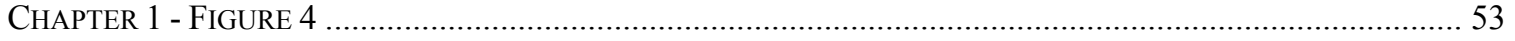

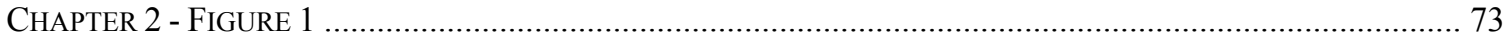

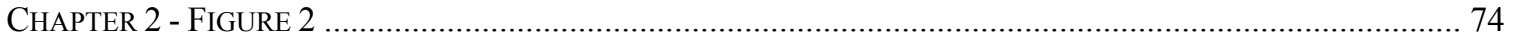

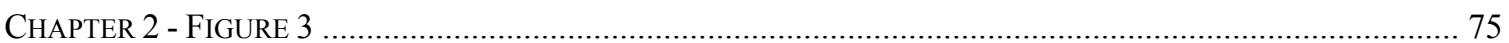

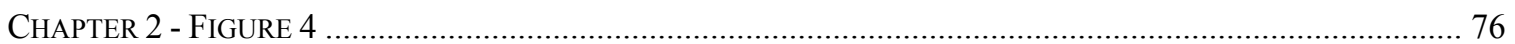

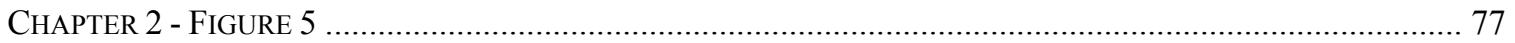

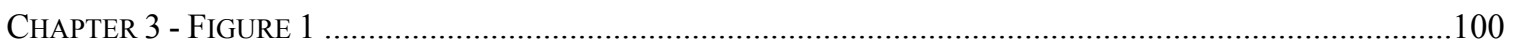

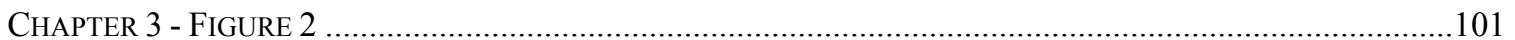

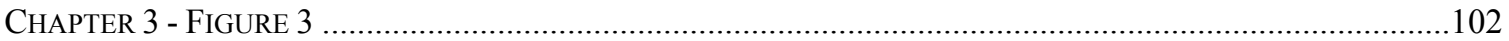

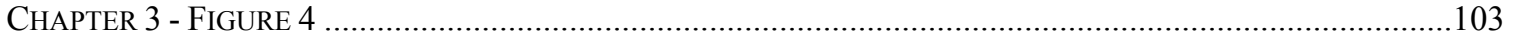

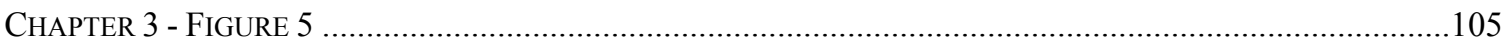

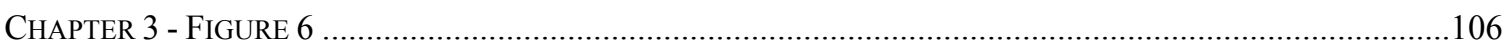

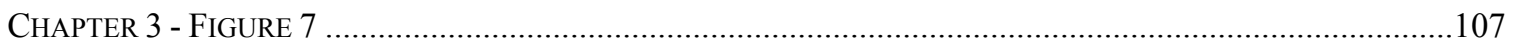

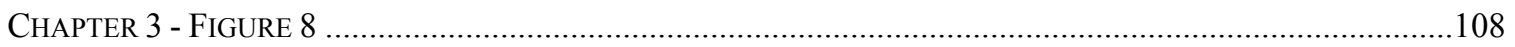

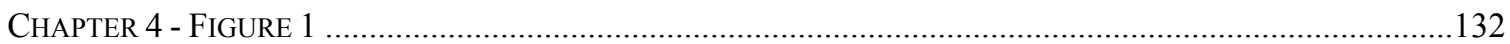

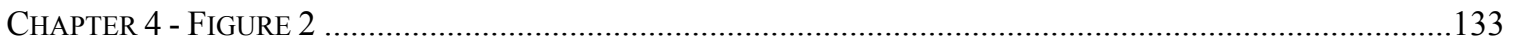

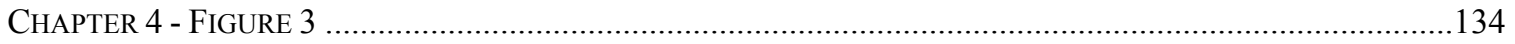

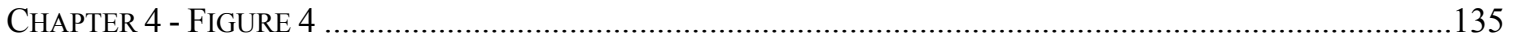

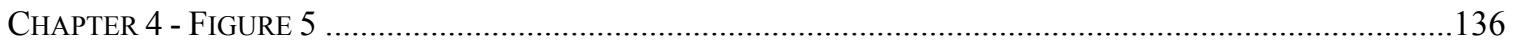

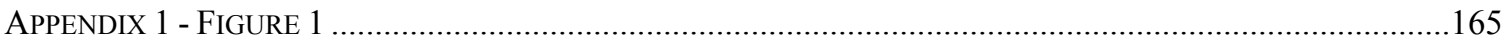

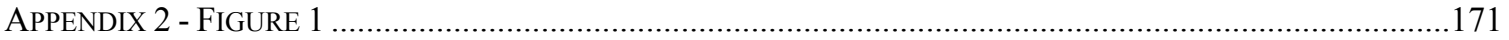




\section{REVIEW OF LITERATURE}

\section{OVERVIEW}

Ergot alkaloids are mycotoxins that can cause ill effects in humans and animals; however, the medical community has also found advantageous uses for these compounds as treatments for some diseases and other clinical conditions. They were first discovered in the sclerotia of the ergot fungus, Claviceps purpurea. Ingestion of grain contaminated with ergot sclerotia causes ergotism (44). Ergot alkaloids have been extensively studied in $C$. purpurea, although the pathway by which ergot alkaloids are synthesized has yet to be fully determined $(21,57)$. Ergot alkaloids also have been reported in other species, such as Neotyphodium spp. which are endophytes of grasses, Penicillium spp. and Aspergillus fumigatus $(15,30)$. A. fumigatus is a common fungus found in a variety of indoor environments, including ventilation systems, and generates airborne spores that are a potential danger to the health of humans. The spores can embed and survive in the lungs of humans and animals, causing ill health to the affected individual and significant lung disease in immunocompromised persons. Whether ergot alkaloids contribute to the virulence of the fungus or cause toxicoses in the absence of fungal colonization is unknown.

\section{ERGOT ALKALOIDS AND THEIR HISTORICAL SIGNIFICANCE}

Ergot alkaloids are a family of indole-derived mycotoxins that were first discovered in the ergot fungus, Claviceps purpurea and are known to cause a wide range of effects on exposed individuals due to their nonselective interactions with several monoamine neurotransmitter receptors. Unnecessary for the survival of the fungus, at least under laboratory conditions, these secondary metabolites clearly impact individuals that 
consume them. Typically these metabolites affect the central nervous, circulatory, immune, and reproductive systems $(20,42,47,58)$. In mammals, they can act as agonists and/ or antagonists of dopamine, 5-hydroxytryptamine (5-HT; serotonin), and noradrenaline (norepinephrine) receptors $(46,47)$. The severity to which humans and animals are affected is dependent upon multiple factors such as the toxicity of the particular ergot alkaloid, exposure time, and the health status of the individual. In the past, people ingested elevated amounts of ergot alkaloids through consumption of grain contaminated with sclerotia of the ergot fungus, C. purpurea (44), which led to ergotism, a condition then known as St. Anthony's fire. Exposure to alkaloids can cause hallucinations, gangrene, and even death. It has been hypothesized that these toxins were responsible for the Salem witch trials in Massachusetts in the seventeenth century (50).

Different types of alkaloids affect the clinical manifestations of ergotism.

However, there is significant overlap in the types of symptoms induced by different ergot alkaloid sources. Ergopeptine alkaloids such as ergotamine or ergocristine, peptide derivatives of lysergic acid, are frequently associated with a gangrenous form of ergotism due to their vasoconstrictive activity. They cause painful edema of the legs and gangrene at the tendons. The last documented outbreak of this form of ergotism was seen in Ethiopia in 1977-1978 where 34\% of the affected individuals died. Ergotism due to intoxication with ergot clavines or simple amides of lysergic acid is more typically associated with a convulsive form of ergotism. Affected individuals experience gastrointestinal symptoms as well as problems within their central nervous system. The last documented outbreak of ergotism due to ergot clavines was seen in India in 1975. All affected individuals survived (44). Today, ergotism in humans is a rare occurrence 
due to grain cleaning before milling which removes most of the sclerotia; however, it is still a major concern for animal husbandry of domestic animals, such as cattle, horses, sheep, pigs, and chickens, which feed on contaminated grasses or grains $(1,16)$.

Lambs fed a diet containing endophyte infected fescue seed $(\mathrm{E}+)$ showed a linear reduction in feed intake, skin temperature, thermocirculation index (TCI), and prolactin concentration when fed diets rich in E+ (17). Prolactin concentration was significantly lowered by E+ intake, presumably due to the presence of ergot alkaloids.

Supplementation of feed with ergovaline caused a similar effect, but not to the same extent as $\mathrm{E}+$, suggesting that the other ergot alkaloids also play a role in toxicoses. In a different study, ergovaline and lysergic acid could be detected in ruminal fluid, urine (lysergic acid only), and feces in lambs fed an E+ diet (10).

Research has shown cattle that graze E+ tall fescue have lowered average daily weight gains, increased body temperatures, rough coats, lowered prolactin production, and reduced conception rates (16). Cattle grazing on mixed grasses containing E+ and Eshowed intermediate weight gain compared to cattle that grazed E- only and E+ only pastures. A Norwegian study reported the occurrence of ergotism in free-living moose and roe deer that fed on wild grasses (23). Ergocristine was the major alkaloid found among the sampled wild grasses; this ergopeptine alkaloid, along with other unknown alkaloids, could account for the gangrenous symptoms in the wild Norwegian cervids (61). Ergot alkaloids have also been shown to affect rabbits, insects, and bacteria $(6,12$, $37,42,48,49)$.

Because of their high affinity for neurotransmitter receptors, there are also advantageous effects of alkaloid consumption. The medical community uses these 
compounds as a treatment for migraines, and they are also effective against Parkinsonism and to induce uterine contractions in childbirth (26). However, because of their lack of specificity for individual neurotransmitter receptors, negative side effects limit the utility of these drugs $(30,42)$. During the twentieth century, a Swiss chemist named Albert Hofmann began creating semi-synthetic ergot alkaloids. Among his many concoctions, arose two important drugs, Methergine, which was responsible for preventing hemorrhage after childbirth, and lysergic acid diethylamide (LSD), a hallucinogenic drug used by psychiatrists and the CIA, as well as a popular recreational drug of the 60's and early 70's (1). Use of LSD or dihydroergotamine has been known to cause a condition known as serotonin syndrome, which is a condition that shares similar clinical features with convulsive ergotism $(11,18)$.

\section{CLASSES OF ERGOT ALKALOIDS}

Ergot alkaloids are often characterized as clavines, simple amides of lysergic acid or ergopeptines (Fig. 1). Clavines are precursors of lysergic acid; whereas both simple amides and ergopeptines, non-ribosomally synthesized peptides, are derivatives of lysergic acid $(41,51)$. Ergot alkaloids are derivatives of tryptophan, and the majority of them contain ergoline, a tetracyclic ring structure, as the basic structure, with methylation at N-6. Modifications at the C-8 position, double bonds between carbon 8 and 9 or 9 and 10 , and opening of ring D are various ways ergot alkaloids differ, causing them to have different chemical properties (29). Substitutions at C-8 separate ergot alkaloids into the three different classes (15)(Fig.1). Clavines are the simplest of the ergot alkaloids. Typically, clavines have a methyl or hydroxymethyl group attached to the C-8 of the ergoline; however some have a carbonyl group at this site (29). Chanoclavine and 
members within the subclass 6,7-secoergolines have an open D ring, which differentiates them from other clavines. Clavines are the ultimate end products of the ergot alkaloid pathway in some fungi (e.g., Claviceps fusiformis and A. fumigatus); however, they accumulate in other ergot alkaloid-producing fungi as products of shunts off the pathway or are intermediates to more complex ergot alkaloids. Besides causing toxicoses in exposed individuals, clavines also affect food preference by acting as a deterrent in rabbit feeding in experiments using endophyte infected grasses (37).

Simple amides of lysergic acid include lysergic acid amide (ergine), ergonovine, lysergic acid-2-hydroxyethylamide, and lysergyl alanine. The observation that lysergyl peptide synthetase 1 is required for synthesis of ergine and lysergyl alanine suggests that these simple amides are by-products of ergopeptine synthesis (43). However, in some cases simple amides accumulate as the ultimate product of the ergot alkaloid pathways, such as ergine and lysergic acid-2-hydroxyethyl amide in C. paspali.

Ergopeptines, the most complex ergot alkaloids, have three amino acids attached to D-lysergic acid. Biosynthesis of ergopeptines requires a peptide synthetase complex to catalyze the peptide bonds between the amino acids and lysergic acid. Most of the variation in ergopeptines comes from the differences in amino acids that occupy positions I and II of the tripeptide chain attached to lysergic acid (Fig. 2). Proline typically occupies amino acid position III; the sole exception being the presence of alanine at this position in ergobalansine (29). Ergopeptines can be divided into three subclasses: the ergotamine group, ergotoxine group, and ergoxine group; classification is dependent on the amino acid present in position I of the peptide. Individual ergot alkaloids within subclasses differ in the amino acid residing in position II. Possible amino acids 
occupying this position are phenylalanine, leucine, isoleucine, norleucine, valine, methionine and alpha-amino butyric acid (51). Ergovaline is a major alkaloid found within Neotyphodium spp., whereas ergosine and ergotamine are examples of abundant ergopeptine within Claviceps spp. $(34,36)$.

Ergopeptams, also known as lactam ergot alkaloids, are non-cyclol ergopeptines, due to the lack of hydroxylation of the amino acid in position I; hence a cyclol bridge is unable to form. The proline in these alkaloids is in D-configuration $(15,29)$. Ergopeptams are further characterized into the subclasses, ergotamams, ergoxams, ergotoxams, and ergoannams.

\section{ERGOT ALKALOID PRODUCING FUNGI}

Claviceps and the Neotyphodium endophytes $(42,51)$ are clavicipitaceous genera belonging to the order Hypocreales. Claviceps spp. parasitize flowers of rye and other cereals; sclerotia, fungal overwintering bodies, replace the infected plant host's seeds. Ergot alkaloid-rich sclerotia may be harvested along with the grain, and so grain cleaning is essential to eliminate toxicoses. Neotyphodium spp. and their teleomorphs, Epichloë spp., are endophytes of cool-season grasses, such as tall fescue and perennial ryegrass (51). These fungi live symbiotically with their host species, grow intercellularly within the plant, and disseminate in seeds $(15,41)$. The fungus provides many benefits to its host, such as drought tolerance and protection from pests. However, the animals that graze on these $\mathrm{E}+$ grasses are susceptible to toxicoses due to mycotoxin production (16, 35, 48). Ergot alkaloids are typically found in the pseudostems and leaf blades of these forages but are either undetectable or only found in extremely low concentrations (relative to pseudostem) within the roots $(40,41)$. These clavicipitaceous fungi have the 
capacity to biosynthesize a wide spectrum of alkaloids, especially lysergic acid amides and ergopeptines $(42,51)$. However, due to the slow growth of these fungi and their dependency on their plant hosts for reliable production of alkaloids, biochemical and molecular aspects of the pathway have been elucidated slowly $(39,63)$.

Several other species, besides those within the genera Claviceps and Neotyphodium, produce ergot alkaloids. These include species within the genera Aspergillus and Penicillium, which are common fungi in the order Eurotiales (30). These fungi produce only the clavine class of ergot alkaloids. Among these is $A$. fumigatus, a thermotolerant opportunistic pathogen. This fungus is capable of growing at temperatures up to $55^{\circ} \mathrm{C}$ and surviving at $70{ }^{\circ} \mathrm{C}$. It colonizes domestic and public buildings, increasing exposure by humans (31). The fungus grows in moist areas, such as basements and ventilation system ducts; it also is ubiquitous outdoors, with higher concentrations in areas surrounding compost and waste removal facilities. The conidia (asexual spores) can become airborne and inhaled by humans and animals, which can infect and cause disease, act as an allergen, cause toxicoses, or cause inflammatory responses (13). Contamination of ventilation systems with A. fumigatus in hospitals poses a serious threat to immune-deficient individuals of acquiring aspergillosis. For the general population, a common question is: are indoor molds truly causing a "new" disease and if so, why is this more of an issue today than in the past? Many studies correlate disease with indoor air quality, specifically regarding mold concentrations, in buildings and homes. However, many of these claims have insufficient evidence and lack consistent clinical diagnoses. Future studies need to correct the flaws of past research to address these questions (56). 
Ergot alkaloid producers within Aspergillus and Penicillium produce clavine ergot alkaloids exclusively (30). Aspergillus fumigatus accumulates four ergot alkaloids at relatively high levels: festuclavine and fumigaclavines A, B, and C (7, 54, 64) (Fig. 3). Many Claviceps spp. and Neotyphodium spp. are known to produce clavines, simple amides of lysergic acid and ergopeptines; however, not all species have the capability to synthesize all three classes, such as $C$. fusiformis, which produces only clavines $(15,51)$.

The ergot alkaloid pathway is inefficient, allowing accumulation of both intermediates and end products (36). Production of a wide spectrum of alkaloids could protect the fungus via deterrence of herbivores. Panaccione et al. (37) showed clavines to be a greater deterrent to rabbit feeding than are ergopeptines. However, ergovaline, an ergopeptine, caused a decrease in appetite. This occurrence is supported by ergovaline's ability to act as an agonist of $5 \mathrm{HT}_{2 \mathrm{~A}}$, which is known to lower appetite (52). Also, ergopeptines and/or lysergic acid amides deterred feeding of black cutworm on endophyte-infected grasses (49). Together these studies show benefits associated with inefficiency in the ergot alkaloid pathway.

\section{ERGOT ALKALOID SYNTHESIS GENE CLUSTERS}

Typically, genes controlling the production of secondary metabolites are clustered within fungal genomes. The driving force behind this phenomenon is unclear. Some theories presented as explanations of this occurrence are that clustering of pathway genes facilitates horizontal transfer of the pathway, specifically the selfish cluster hypothesis (62), and that clustering allows coregulation of the secondary metabolite gene cluster (28). The selfish gene hypothesis suggests that genes lacking beneficial qualities to their host persist via horizontal transmission (32). The "selfish cluster" hypothesis takes this 
concept one step further stating that clusters exist because they possess a selective advantage over the alternative situation in which the genes are dispersed in the genome (62). This advantage is independent of any selective advantage as a result of the product biosynthesized by the genes of the cluster. This commentary suggests that not only is the secondary metabolite pathway under selective pressure, but there is also selective pressure acting on the maintenance of the gene cluster arrangement at a subgenomic level. Horizontal transmission could potentially drive the clustering of genes and seems to be more prevalent in eukaryotes with more accessible genomes, such as fungi $(19,32$, $62)$.

Since the biosynthesis of secondary metabolites often is regulated by trans-acting factors, coregulation by cis-acting factors may not seem like a plausible cause for clustering. However, by means of regulating the chromatin environment, coregulation could possibly be a driving force of clustering. LaeA, a nuclear protein found in filamentous fungi, transcriptionally regulates gene clusters; it is thought to act as a methyltransferase capable of regulating transcription of clustered genes via modifying chromatin structure in the cluster (3). Since clustering is a characteristic of secondary metabolite genes, LaeA, could be considered a global regulator of secondary metabolism in filamentous fungi, such as Aspergillus spp. $(2,3)$. LaeA regulates the sterigmatocystin, penicillin, and terrequinone A gene clusters in A. nidulans and gliotoxin and ergot alkaloid gene clusters in A. fumigatus $(2,45)$.

Ergot alkaloid biosynthesis genes are clustered in all of the fungi studied to date (21). Dimethylallyltryptophan (DMAT) synthase catalyzes the determinant step of the ergot alkaloid biosynthesis pathway by forming DMAT from tryptophan and 
dimethylallylpyrophosphate (63). The gene $d m a W$ encodes this enzymatic step, as determined by expression analyses of the C. fusiformis gene in yeast (57) and by gene knockout in Neotyphodium sp. (63).

Another gene involved in the clavicipitaceous pathway, $\operatorname{lps} A$, a neighboring gene of $d m a W$ in the Claviceps genome, was functionally analyzed by gene knockout in Neotyphodium sp. Lp1 (39). Structural analysis of $\operatorname{lps} A$ indicates similarities in sequences within modules correspond with same amino acid substrate within certain positions of the polypeptide, whereas sequences that lack similarity correspond with the addition of different amino acids within those positions $(9,21)$. Neotyphodium coenophialum has two different copies of $\operatorname{lps} A$, which corresponds to it being a hybrid of multiple species (9). Biochemical characterization of the $\operatorname{lps} A$ knockout resulted in placing ergine and lysergyl-alanine after lysergyl peptide synthetase in ergot alkaloid biosynthesis (43).

A second peptide synthetase, encoded by $\operatorname{lps} B$, plays a role in ergopeptine synthesis, based on knockout analysis in C. purpurea (8) and in N. lolii (14). Fleetwood et al. (14) found evidence to support the presence of transposons within the N. lolii ergot alkaloid synthesis (eas) cluster. The eas genes are highly expressed in planta, but were minimally expressed or undetectable in culture (14).

CloA in C. purpurea is responsible for bridging two classes of ergot alkaloids, converting clavines (elymoclavine) to D-lysergic acid (22). Since $C$. fusiformis and $C$. purpurea have different ergot alkaloid profiles, genomic comparison of the eas gene cluster between these species was performed to determine the genetic differences responsible for the corresponding chemotypes. Both clusters contain nine eas gene 
homologues, including $\operatorname{cloA}$ and lpsB, both of which are involved in biosynthesis of ergopeptines. All nine eas genes were expressed in C.fusiformis. However, CloA and LpsB were enzymatically inactive, and therefore C. fusiformis is unable to convert clavines to ergopeptines (33). These genes involved in ergot alkaloid biosynthesis are clustered in the genome of $C$. purpurea $(21,59)$. Several additional genes clustered around $\operatorname{dmaW}, \operatorname{lps} A, \operatorname{lps} B$, and $\operatorname{clo} A$ have functions typical of genes involved in secondary metabolism.

\section{SECONDARY METABOLISM AND SPORULATION}

There is an apparent association between morphological differentiation and secondary metabolism in microorganisms $(5,25)$. Hopwood (25) demonstrated that sporulation and secondary metabolite biosynthesis in the filamentous bacterium, Streptomyces coelicolor A3, was translationally regulated by $b l d A$, a gene that encodes for a tRNA corresponding to a leucine codon. The mRNA involved in vegetative growth does not require this codon during translation, but it is essential for formation of proteins responsible for differential growth and antibiotic synthesis. Typically in fungi, secondary metabolites associate with sporulation either as activators of sporulation, pigments necessary for sporulation structures, or as toxic metabolites whose production coincides with sporulation in the growing organism (5). Environmental conditions are similar for secondary metabolite synthesis and sporulation; environmental factors such as, $\mathrm{pH}$, temperature, air-surface interface, and nutrient supply have been found to regulate both sporulation and secondary metabolite production in Aspergillus spp. (5).

Studies functionally analyzing genes regulating sporulation also have indicated a corresponding involvement in mycotoxin production in fungi. In Aspergillus spp., 
asexual sporulation and mycotoxin biosynthesis are regulated by a common G-protein signaling pathway (4). In A. nidulans, FadA is responsible for vegetative growth through activation of cyclic AMP-dependent protein kinase A (PkaA). FadA activation or knock out of $f l b A$ inhibits the production of conidia and sterigmatocystin (ST), a precursor of aflatoxin biosynthesis (24). In the presence of FlbA, the FadA pathway is inhibited, at least partially, which allows ST synthesis and asexual sporulation to occur. FlbA is a protein with a RGS (Regulator of G protein Signaling) domain. Proteins in this family negatively regulate G-protein mediated pathways (24). Mutations in the sec gene within A. parasiticus greatly reduce conidiophore production, and also eliminate aflatoxin biosynthesis and expression of $a f l R$, a regulatory gene involved in the aflatoxin pathway (27). Knockout analysis of the regulatory gene, stuA, in A. fumigatus again demonstrates the relationship between conidiation and secondary metabolism. The transcription factor, StuAp, is responsible for proper conidiophore development and regulation of potentially six secondary metabolite clusters, one being ergot alkaloid biosynthesis $(53,60)$. This result exemplifies the apparent association between sporulation and secondary metabolism, both of which are governed by a complex regulatory pathway. 


\section{ERGOT ALKALOIDS IN ASPERGILLUS FUMIGATUS}

Spilsbury and Wilkinson (55) were the first to document production of the ergot alkaloid, festuclavine, in A. fumigatus. They also characterized two novel festuclavine derivatives, fumigaclavines A and B, produced by this fungus. Soon after, Yamano et al. (64) noted an additional ergot alkaloid, fumigaclavine $\mathrm{C}$, was also synthesized by $A$. fumigatus . Years later, Cole et al. (7) further characterized fumigaclavine $\mathrm{C}$ using broth cultures of A. fumigatus that had been isolated from moldy silage.

More recently Panaccione and Coyle (38) demonstrated that $A$. fumigatus accumulates ergot alkaloids in association with its asexual spores (conidia), although exact location has not been discerned. Accumulated ergot alkaloids were fumigaclavine $\mathrm{C}$, festuclavine, fumigaclavine $\mathrm{A}$, and fumigaclavine $\mathrm{B}$ in the order of abundance (Appendix 1, Table 2). Under environmentally relevant conditions, the total mass of ergot alkaloids often exceeded $1 \%$ of the mass of a conidium. Extraction of ergot alkaloids from conidia of $A$. fumigatus cultured on various media, revealed that extracts from cultures grown on latex paint or cultured maize seedlings accumulated the greatest quantities of ergot alkaloids. Important physical attributes of conidia likely to affect their respirability, i.e. diameter, mass, and specific gravity, were significantly lower in $A$. fumigatus when compared to Aspergillus nidulans, Aspergillus niger, and Stachybotrys chartarum (Appendix 1, Table 1).

\section{OBJECTIVES AND POTENTIAL IMPACT OF CURRENT STUDY}

In efforts to further understand ergot alkaloid biosynthesis, the objectives of this study are to: 
1. Characterize the ergot alkaloids produced by A. fumigatus, complete a structural analysis of the genes within the pathway

2. Functionally analyze ergot alkaloid synthesis genes via gene knockouts, determine the spectrum of ergot alkaloids produced by each mutant strain and compare it to the production of the wild type

3. Investigate the association of sporulation and ergot alkaloid production in $A$. fumigatus.

Several of the early steps in ergot alkaloid biosynthesis are just hypothesized and through this investigation they may be characterized experimentally. Analysis of the gene cluster in A. fumigatus will aid in identifying genes involved in ergot alkaloid production in other species, especially genes involved in the early steps of the pathway thought to be shared by multiple species. Ergot alkaloids are important metabolites in agriculture and medicine. These metabolites possess a number of beneficial qualities, despite their many negative side effects. Characterization of the ergot alkaloid pathway and the ability to control the spectrum of alkaloids produced may lead to advances in these fields by providing a potential means of eliminating the negative side effects. Likewise, production of alkaloids could be associated with cause of illness when $A$. fumigatus infects an individual. Mutant strains developed from this research could be used for testing effects of ergot alkaloids on virulence and determining their role in mycoses and mycotoxicoses. 


\section{REFERENCES}

1. Bennett, J. W., and M. Klich. 2003. Mycotoxins. Clin. Microbiol. Rev. 16:497-516.

2. Bok, J., D. Hoffmeister, L. Maggio-Hall, R. Murillo, J. Glasner, and N. Keller. 2006. Genomic mining for Aspergillus natural products. Chem. Biol. 13:31-37.

3. Bok, J. W., and N. P. Keller. 2004. LaeA, a regulator of secondary metabolism in Aspergillus spp. Euk. Cell 3:527-535.

4. Brodhagen, M., and N. P. Keller. 2006. Signalling pathways connecting mycotoxin production and sporulation Mol. Plant Path. 7:285-301.

5. Calvo, A. M., R. A. Wilson, J. W. Bok, and N. P. Keller. 2002. Relationship between secondary metabolism and fungal development. Microbiol. Mol. Biol. Rev. 66:447-459.

6. Clay, K., and C. Schardl. 2002. Evolutionary origins and ecological consequences of endophyte symbiosis with grasses. Am. Nat. 160:S199-S127.

7. Cole, R. J., J. W. Kirksey, J. W. Dorner, D. M. Wilson, J. C. Johnson, A. N. Johnson, D. M. Bedell, J. P. Springer, and K. K. Chexal. 1977. Mycotoxins produced by Aspergillus fumigatus species isolated from molded silage. J. Agric. Food Chem. 25:826-830.

8. Correia, T., N. Grammel, I. Ortel, U. Keller, and P. Tudzynski. 2003. Molecular cloning and analysis of the ergopeptine assembly system in the ergot fungus Claviceps purpurea. Chem. Biol. 10:1281-1292. 
9. Damrongkool, P., A. B. Sedlock, C. A. Young, R. D. Johnson, K. E. Goetz, B. Scott, C. L. Schardl, and D. G. Panaccione. 2005. Structural analysis of a peptide synthetase gene required for ergopeptine production in the endophytic fungus Neotyphodium lolii. DNA Sequ. 16:379-385.

10. De Lorme, M. J. M., S. L. Lodge-Ivey, and A. M. Craig. 2007. Physiological and digestive effects of Neotyphodium coenophialum-infected tall fescue fed to lambs. J. Anim. Sci. 85:1199-1206.

11. Eadie, M. J. 2003. Convulsive ergotism: epidemics of the serotonin syndrome? Lancet Neurol. 2:429-434.

12. Eich, E., and H. Pertz. 1999. Antimicrobial and antitumor effects of ergot alkaloids and their derivatives, p. 411-440. In V. Kren and L. Cvak (ed.), Ergot: The Genus Claviceps. Harwood Academic Publishers, The Netherlands.

13. Fischer, G., and W. Dott. 2003. Relevance of airborne fungi and their secondary metabolites for environmental, occupational and indoor hygiene. Arch. Microbiol. 179:75-82.

14. Fleetwood, D. J., B. Scott, G. A. Lane, A. Tanaka, and R. D. Johnson. 2007. A complex ergovaline gene cluster in Epichloë endophytes of grasses. Appl. Environ. Microbiol. 73:2571-2579.

15. Flieger, M., M. Wurst, and R. Shelby. 1997. Ergot alkaloids -- sources, structures and analytical methods. Folia Microbiol. 42:3-30.

16. Fribourg, H. A., and J. C. Waller. 2005. Neotyphodium research and application in the USA, p. 3-22. In C. A. Roberts, C. P. West, and D. E. Spiers (ed.), 
Neotyphodium in cool-season grasses, 1st ed. Blackwell Publishing Professional, Ames, Iowa.

17. Gadberry, M. S., T. M. Denard, D. E. Spiers, and E. L. Piper. 2003. Effects of feeding ergovaline on lamb performance in a heat stress environment. J. Anim. Sci. 81:1538-1545.

18. Ganetsky, M., and D. E. Brush. 2005. Serotonin syndrome-What have we learned? Clin. Ped. Emerg. Med. 6:103-108.

19. Goddard, M. R., and A. Burt. 1999. Recurrent invasion and extinction of a selfish gene. Proc. Natl. Acad. Sci. USA 96:13880-13885.

20. Gröger, D., and H. G. Floss. 1998. Biochemistry of ergot alkaloids achievements and challenges. Alkaloids 50:171-218.

21. Haarmann, T., C. Machado, Y. Lübbe, T. Correia, C. L. Schardl, D. G. Panaccione, and P. Tudzynski. 2005. The ergot alkaloid gene cluster in Claviceps purpurea: extension of the cluster sequence and intra species evolution. Phytochemistry 66:1312-1320.

22. Haarmann, T., I. Ortel, P. Tudzynski, and U. Keller. 2006. Identification of the cytochrome P450 monooxygenase that bridges the clavine and ergoline alkaloid pathways. ChemBioChem 7:645-652.

23. Handeland, K., and T. Vikoren. 2005. Presumptive gangrenous ergotism in free-living moose and a roe deer. J. Wildl. Dis. 41:636-642.

24. Hicks, J. K., J.-H. Yu, N. P. Keller, and T. H. Adams. 1997. Aspergillus sporulation and mycotoxin production both require inactivation of the FadA G protein-dependent signaling pathway. EMBO J. 16:4916-4923. 
25. Hopwood, D. A. 1988. The Leeuwenhoek lecture, 1987. Towards an understanding of gene switching in Streptomyces, the basis of sporulation and antibiotic production. Proc. R. Soc. Lond. Biol. 235:121-138.

26. Houghton, P. J., and M.-J. Howes. 2005. Natural products and derivatives affecting neurotransmission relevant to Alzheimer's and Parkinson's disease. Neurosignals 14:6-22.

27. Kale, S. P., J. W. Cary, D. Bhatnagar, and J. W. Bennett. 1996. Characterization of experimentally induced, nonaflatoxigenic variant strains of Aspergillus parasiticus. Appl. Environ. Microbiol. 62:3399-3404.

28. Keller, N. P., and T. M. Hohn. 1997. Metabolic pathway gene clusters in filamentous fungi. Fungal Genet. Biol. 21:17-29.

29. Keller, U. 1999. Biosynthesis of ergot alkaloids, p. 95-163. In V. Kren and L. Cvak (ed.), Ergot: The Genus Claviceps. Harwood Academic Publishers, The Netherlands.

30. Kozlovsky, A. G. 1999. Producers of ergot alkaloids out of Claviceps genus, p. 479-499. In V. Kren and L. Cvak (ed.), In Ergot: The Genus Claviceps. Harwood Academic Publishers, The Netherlands.

31. Latge, J.-P. 1999. Aspergillus fumigatus and Aspergillosis. Clin. Microbiol. Rev. 12:310-350.

32. Lawrence, J. G., and J. R. Roth. 1996. Selfish operons: horizontal transfer may drive the evolution of gene clusters. Genetics 143:1843-1860.

33. Lorenz, N., E. V. Wilson, C. Machado, C. L. Schardl, and P. Tudzynski. 2007. Comparison of ergot alkaloid biosynthesis gene clusters in Claviceps 
species indicates loss of late pathway steps in evolution of C. fusiformis. Appl. Environ. Microbiol. 73:7185-7191.

34. Lyons, P. C., R. D. Plattner, and C. W. Bacon. 1986. Occurrence of peptide and clavine ergot alkaloids in tall fescue grass Science 232:487-489.

35. Malinowski, D. P., D. P. Belesky, and G. C. Lewis. 2005. Abiotic stresses in endophytic grasses, p. 187-199. In C. P. W. C. A. Roberts, and D. E. Spiers (ed.), Neotyphodium in cool-season grasses, 1 ed. Blackwell Publishing Professional, Ames, Iowa.

36. Panaccione, D. G. 2005. Origins and significance of ergot alkaloid diversity in fungi. FEMS Microbiol. Lett. 251:9-17.

37. Panaccione, D. G., J. R. Cipoletti, A. B. Sedlock, K. P. Blemings, C. L. Schardl, C. Machado, and G. E. Seidel. 2006. Effects of ergot alkaloids on food preference and satiety in rabbits, as assessed with gene-knockout endophytes in perennial ryegrass (Lolium perenne). J. Agric. Food Chem. 54:4582-4587.

38. Panaccione, D. G., and C. M. Coyle. 2005. Abundant respirable ergot alkaloids from the common airborne fungus Aspergillus fumigatus. Appl. Environ. Microbiol. 71:3106-3111.

39. Panaccione, D. G., R. D. Johnson, J. Wang, C. A. Young, P. Damrongkool, B. Scott, and C. L. Schardl. 2001. Elimination of ergovaline from a grassNeotyphodium endophyte symbiosis by genetic modification of the endophyte. Proc. Natl. Acad. Sci. USA 98:12820-12825.

40. Panaccione, D. G., J. B. Kotcon, C. L. Schardl, R. J. Johnson, and J. B. Morton. 2006. Ergot alkaloids are not essential for endophytic fungus- 
associated population suppression of the lesion nematode, Pratylenchus scribneri, on perennial ryegrass. Nematology 8:583-590.

41. Panaccione, D. G., C. Schardl, and C. M. Coyle. 2006. Pathways to diverse ergot alkaloid profiles in fungi, p. 23-52 In J. T. Romeo (ed.), Recent Advances in Phytochemistry, vol. 40. Elsevier, Amsterdam, The Netherlands.

42. Panaccione, D. G., and C. L. Schardl. 2003. Molecular genetics of ergot alkaloid biosynthesis, p. 399-424. In J. F. W. Jr., C. W. Bacon, N. L. HywelJones, and J. W. Spatafora (ed.), The Clavicipitalean Fungi: Evolutionary Biology, Chemistry, Biocontrol, and Cultural Impacts. Marcel-Dekker, New York.

43. Panaccione, D. G., B. A. Tapper, G. A. Lane, E. Davies, and K. Fraser. 2003. Biochemical outcome of blocking the ergot alkaloid pathway of a grass endophyte. J. Agric. Food Chem. 51:6429-6437.

44. Peraica, M., B. Radic, A. Lucic, and M. Pavlovic. 1999. Toxic effects of mycotoxins in humans. Bull. World Health Org. 77:754-766.

45. Perrin, R. M., N. D. Fedorova, J. W. Bok, R. A. Cramer, J. R. Wortman, H. S. Kim, W. C. Nierman, and N. P. Keller. 2007. Transcriptional regulation of chemical diversity in Aspergillus fumigatus by LaeA. PLOS Pathogens 3:508517.

46. Pertz, H. 1996. Naturally occurring clavines: antagonism/partial agonism at 5HT2A receptors and antagonism at $\alpha 1$-adrenoceptors in blood vessels. Planta Med. 62:387-392. 
47. Pertz, H., and E. Eich. 1999. Ergot alkaloids and their derivatives as ligands for serotoninergic, dopaminergic, and adrenergic receptors, p. 411-440. In V. Kren and L. Cvak (ed.), Ergot: The Genus Claviceps. Harwood Academic Publishers, The Netherlands.

48. Popay, A. J., and S. A. Bonos. 2005. Biotic responses in endophytic grasses, p. 163-185. In C. P. W. C. A. Roberts, and D. E. Spiers (ed.), Neotyphodium in cool-season grasses, 1st ed. Blackwell Publishing Professional, Ames, Iowa.

49. Potter, D. A., J. T. Stokes, C. T. Redmond, C. L. Schardl, and D. G.

Panaccione. 2008. Contribution of ergot alkaloids to suppression of a grassfeeding caterpillar assessed with gene knockout endophytes in perennial ryegrass. Entomol. Experiment. Applic. 126:138-147.

50. Rusyniak, D. E., R. B. Furbee, and R. Pascuzzi. 2005. Historical neurotoxins: What we have learned from toxins of the past about diseases of the present. Neurol. Clin. 23:337-352.

51. Schardl, C., D. G. Panaccione, and P. Tudzynski. 2006. Ergot alkaloids-biology and molecular biology, p. 45-86. In G. A. Cordell (ed.), Alkaloids Chem. Biol., vol. 63. Academic Press, San Diego, CA.

52. Schöning, C., M. Flieger, and H. H. Pertz. 2001. Complex interaction of ergovaline with 5-HT2A, 5-HT1B/1D, and alpha1 receptors in isolated arteries of rat and guinea pig. J. Anim. Sci. 79:2202-2209.

53. Sheppard, D. C., T. Doedt, L. Y. Chiang, H. S. Kim, D. Chen, W. C. Nierman, and S. G. Filler. 2005. The Aspergillus fumigatus StuA protein 
governs the up-regulation of a discrete transcriptional program during the acquisition of developmental competence. Mol. Biol. Cell 16:5866-5879.

54. Spilsbury, J. F., and S. Wilkinson. 1961. The isolation of festuclavine and two new clavine alkaloids from Aspergillus fumigatus Fres. J. Chem. Soc 5:20852091.

55. Spilsbury, J. F., and S. Wilkinson. 1961. The isolation of festuclavine and two new clavine alkaloids from Aspergillus fumigatus Fres. J. Chem. Soc. 5:20852091.

56. Terr, A. I. 2004. Are indoor molds causing a new disease? J. Allergy Clin. Immun. 113:221-226

57. Tsai, H.-F., H. Wang, J. C. Gebler, C. D. Poulter, and C. L. Schardl. 1995. The Claviceps purpurea gene encoding dimethylallyltryptophan synthase, the committed step for ergot alkaloid biosynthesis. Biochem. Bioph. Res. Co. 216:119-125.

58. Tudzynski, P., T. Correia, and U. Keller. 2001. Biotechnology and genetics of ergot alkaloids. Appl. Microbiol. Biotechnol. 57:593-605.

59. Tudzynski, P., K. Holter, T. Correia, C. Arntz, N. Grammel, and U. Keller. 1999. Evidence for an ergot alkaloid gene cluster in Claviceps purpurea. Mol. Gen. Genet. 261:133-141.

60. Twumasi-Boateng, K., Y. Yu, D. Chen, F. N. Gravelat, W. C. Nierman, and D. C. Sheppard. 2009. Transcriptional profiling identifies a role for BrlA in the response to nitrogen depletion and for StuA in the regulation of secondary metabolite clusters in Aspergillus fumigatus. Euk. Cell 8:104-115. 
61. Uhlig, S., T. Vikøren, L. Ivanova, and K. Handeland. 2007. Ergot alkaloids in Norwegian wild grasses: A mass spectrometric approach. Rapid Commun. Mass Sp. 21:1651-1660.

62. Walton, J. D. 2000. Horizontal gene transfer and the origin of secondary metabolite gene clusters in fungi: An hypothesis. Fungal Genet. Biol. 30:167171.

63. Wang, J., C. Machado, D. G. Panaccione, H.-F. Tsai, and C. L. Schardl. 2004. The determinant step in ergot alkaloid biosynthesis by an endophyte of perennial ryegrass. Fungal Genet. Biol. 41:189-198.

64. Yamano, T., K. Kishino, S. Yamantodani, and M. Abe. 1962. Investigation on ergot alkaloids found in cultures of Aspergillus fumigatus. Takeda Kenkyusho Nempo (Annual Report Takeda Research Laboratories) 21:95-101. 


\section{FIGURE LEGENDS}

Figure 1: Representatives of the three classes of ergot alkaloids. Festuclavine exemplifies clavine ergot alkaloids, ergine is a simple amide of lysergic acid and ergotamine represents ergopeptines. One major difference between these classes is the substitution on C-8 (blue) and location of the double bond (red). The letters, A-D, labeled within ergine, identify the four rings common to ergot alkaloids. The atoms within this four ring structure are numbered 1-15 within ergine.

Figure 2: Amino acids involved in ergopeptine synthesis. Ergopeptines are synthesized by addition of three amino acids onto D-lysergic acid. Typically, proline is one of the three amino acids, therefore differentiation between the ergopeptines is due to the specific amino acids that occupy positions R1 and R2. The chart shows the amino acids involved in assembly of six common ergopeptines.

Figure 3: Overview of hypothesized ergot alkaloid pathways of $A$. fumigatus (red branch) and clavicipitaceous fungi (blue branch). Festuclavine, and fumigaclavine A, $\mathrm{B}$, and $\mathrm{C}$ accumulate in A. fumigatus, with fumigaclavine $\mathrm{C}$ being the ultimate product. DMAPP = dimethylallylpyrophosphate; $\mathrm{PPO}=$ pyrophosphate $; \operatorname{Trp}=$ tryptophan; DMAT $=$ dimethylallyltryptophan. 


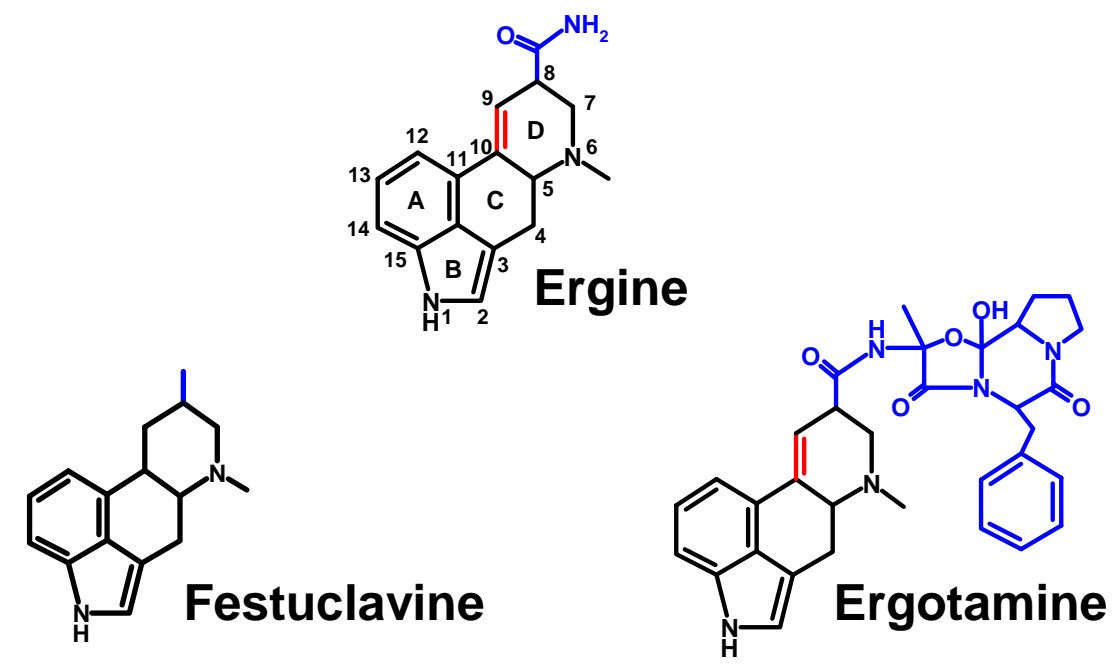

Review of Literature - Figure 1 


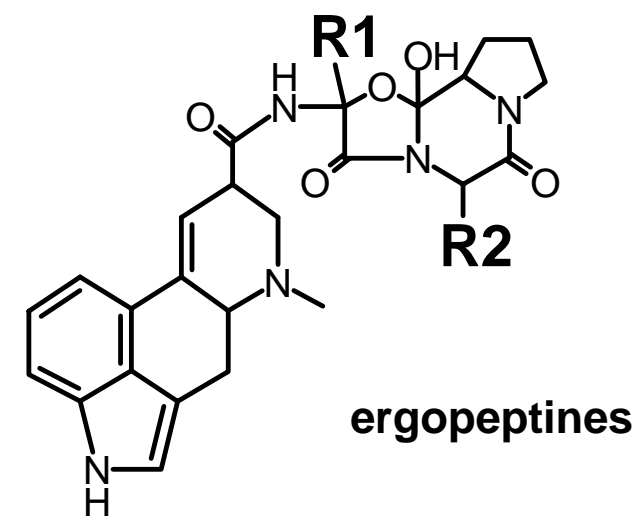

R1 = side chain for:

\begin{tabular}{|c|c|c|c|}
\hline & & ala & val \\
\hline 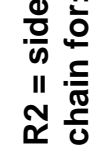 & $\begin{array}{l}\text { val } \\
\text { leu } \\
\text { phe }\end{array}$ & $\begin{array}{l}\text { ergovaline } \\
\text { ergosine } \\
\text { ergotamine }\end{array}$ & $\begin{array}{l}\text { ergocornine } \\
\alpha \text {-ergocryptine } \\
\text { ergocristine }\end{array}$ \\
\hline
\end{tabular}

Review of Literature - Figure 2 


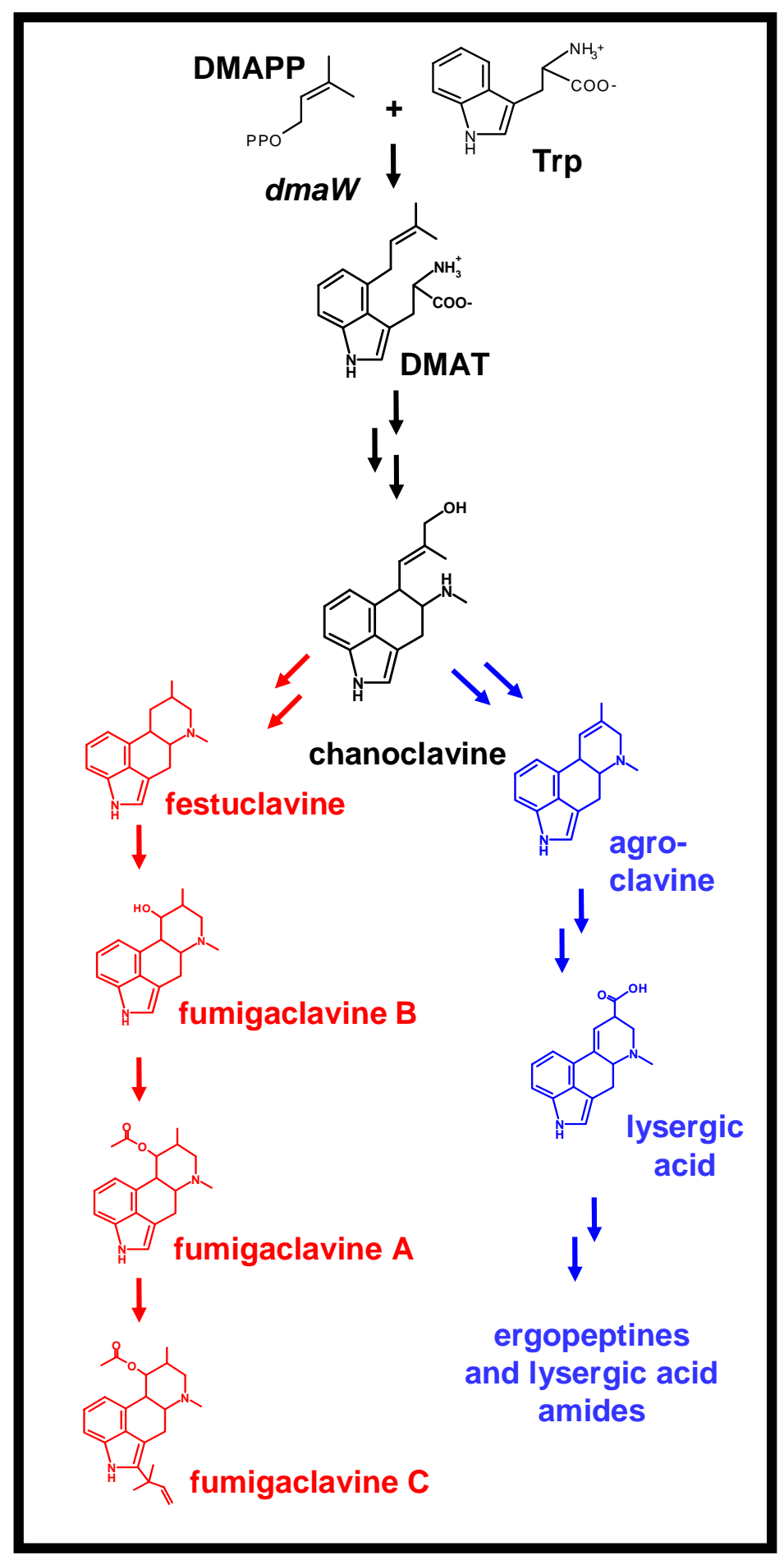

Review of Literature - Figure 3 


\title{
CHAPTER 1: An ergot alkaloid biosynthesis gene and clustered hypothetical genes from Aspergillus fumigatus
}

Published as: Coyle, C. M., and D. G. Panaccione. 2005. Appl. Environ. Microbiol. 71:3112-3118.

\begin{abstract}
Ergot alkaloids are mycotoxins that interact with several monoamine receptors, negatively affecting cardiovascular, nervous, reproductive, and immune systems of exposed humans and animals. Aspergillus fumigatus, a common airborne fungus and opportunistic human pathogen, contains at least four ergot alkaloids associated with its conidia. The A. fumigatus genome contains a gene encoding dimethylallyltryptophan (DMAT) synthase, the enzyme that catalyzes the first committed step in ergot alkaloid biosynthesis. Knockout of the DMAT synthase gene eliminated the ergot alkaloids from conidia, and complementation of the mutation restored ergot alkaloid production. Clustered with the DMAT synthase-encoding gene are sequences corresponding to five genes previously proposed to encode steps in the ergot alkaloid pathway of Claviceps purpurea, as well as additional sequences whose deduced protein products are consistent with involvement in the ergot alkaloid pathway. The data indicate a common, but ancient, origin of ergot alkaloid biosynthetic capability with the ergot alkaloid producers in the distantly related Clavicipitaceae.
\end{abstract}




\section{INTRODUCTION}

Ergot alkaloids are a complex family of indole-derived mycotoxins with varied and significant biological activities $(6,7,14,25)$. Fungi derived from two relatively divergent ascomycete orders (Hypocreales and Eurotiales) produce ergot alkaloids. Several fungi in the family Clavicipitaceae (order Hypocreales) including the ergot fungi in the genus Claviceps and certain grass endophytes in the genera Epichloë (including their imperfect relatives in the genus Neotyphodium) and Balansia produce ergot alkaloids in association with their grass hosts $(3,6,7,14,25)$. The rather distantly related imperfect fungus Aspergillus fumigatus, a common saprophyte and opportunistic human pathogen with close relatives in the order Eurotiales, produces ergot alkaloids in broth culture $(4,23)$ and in association with its airborne conidia (13).

Ergot alkaloid-producing members of the Clavicipitaceae (clavicipitaceous fungi) produce a wide variety of clavine and lysergyl-derived ergot alkaloids but commonly accumulate ergopeptines (nonribosomally synthesized peptides containing lysergic acid and three amino acids) or simple amides of lysergic acid such as ergine and ergonovine $(6,7,8,14)$. Aspergillus fumigatus produces several clavine ergot alkaloids including festuclavine and fumigaclavines A, B, and C $(4,13,23)$. Most of the clavine and lysergyl-derived ergot alkaloids contain the same four-membered ergoline ring system but differ in the number, type, and position of side chains $(6,7,8,14)$.

Festuclavine, one of the ergot alkaloids produced by A. fumigatus, was first described from C. purpurea $(6,7)$ and also has been detected in Neotyphodium spp. (15, 19). However, none of the fumigaclavines produced by A. fumigatus has been detected in a member of the Clavicipitaceae. Likewise the end products of the Claviceps spp. and 
Neotyphodium spp. pathways (ergopeptines and lysergic acid amides) have not been found in A. fumigatus. This distribution of alkaloids is consistent with a hypothesis that early steps of the ergot alkaloid pathway are shared by these diverse fungal species but that the later portions of the pathway differ between A. fumigatus and the clavicipitaceous fungi.

The ergot alkaloid pathway is relatively long and may have alternate branches or spurs in some ergot alkaloid producers $(7,8,14,15)$. Functions of three genes involved in the ergot alkaloid pathway have been demonstrated by gene knockout. The gene ( $\mathrm{maW}$ ) encoding dimethylallyltryptophan synthase (DMAT synthase) was knocked out in Neotyphodium sp. Lp1, an endophyte of perennial ryegrass (29). Loss of all ergot alkaloids in this knockout mutant indicated that $\mathrm{dmaW}$ controls the determinant step in the ergot alkaloid pathway. A gene encoding lysergyl peptide synthetase 1 (LPS1), one of two components of the lysergyl peptide synthetase complex responsible for assembly of ergopeptines from lysergic acid and three amino acids $(20,28)$, was knocked out in Neotyphodium sp. Lp1 (16). LPS1-deficient knockout mutants failed to produce ergopeptines and simple amides of lysergic acid but still produced clavine ergot alkaloids $(15,16)$. A gene encoding lysergyl peptide synthetase 2 (LPS2), the lysergic acidactivating component of the lysergyl peptide synthetase complex, has been cloned and knocked out in C. purpurea, resulting in the loss of ergopeptines from the knockout mutant (5).

Genes encoding DMAT synthase, LPS1, and LPS2 are clustered within $\sim 32 \mathrm{~kb}$ of each other in the genome of $C$. purpurea $(5,25)$. Moreover, at least six additional genes that encode activities likely to be required for ergot alkaloid production are interspersed 
in this cluster with the ergot alkaloid biosynthesis genes $(5,25,26)$. Analysis of mRNAs from five of the clustered genes indicate they are expressed in cultures grown under conditions conducive to ergot alkaloid production but not under repressive conditions (5). Such clustering and common regulation of secondary metabolite biosynthetic pathway genes is typical of fungal genomes $(9,27)$.

Our objective in this study was to investigate whether the ergot alkaloids of $A$. fumigatus share a common biosynthetic origin with those of the clavicipitaceous fungi by identification and functional analysis of the gene $(\mathrm{dmaW})$ controlling the determinant step in the pathway and through comparison of sequences flanking dmaW in A. fumigatus and C. purpurea.

\section{MATERIALS AND METHODS}

Fungi and culture conditions. Aspergillus fumigatus isolate WVU1943 from parakeet lung was described previously (13) and was manipulated in a class II biosafety cabinet. Isolates were maintained on potato dextrose agar [PDA; $20 \mathrm{~g} / \mathrm{L}$ dehydrated mashed potatoes (Idaho Spuds, Pillsbury, Minneapolis, MN), 20 g/L glucose, 15 g/L agar (BactoAgar, Difco, Detroit, MI)] at room temperature or at $37^{\circ} \mathrm{C}$. Cultures for analysis of ergot alkaloids were grown on PDA at $37{ }^{\circ} \mathrm{C}$. For the preparation of DNA or protoplasts, the fungus was cultured overnight in $15 \mathrm{~mL}$ of potato dextrose broth (Difco) in a deep Petri dish (20-mm depth, 100-mm diameter; Fisher Scientific, Pittsburgh, PA) in an orbital shaker set at $37^{\circ} \mathrm{C}$ and $150 \mathrm{rpm}$. Cultures were inoculated by lightly dusting the medium with conidia that had adhered to a Petri dish lid formerly covering a mature culture. In mock inoculations, an average of $2.1 \times 10^{5}$ conidia per $\mathrm{cm}^{2}$ was deposited by this inoculation method. 


\section{Analysis of the A. fumigatus genome for ergot alkaloid biosynthesis genes.}

Aspergillus fumigatus preliminary sequence data were obtained from The Institute for Genomic Research website at http://www.tigr.org. All A. fumigatus sequences described in this paper are located on assembly 92 (long arm of chromosome 2 , formerly assembly 72 ) between nucleotides $2,894,958$ to $2,926,335$. The presence of sequences in the $A$. fumigatus genome that are similar to known or putative ergot alkaloid biosynthesis genes was assessed by application of the tblastn algorithm (1) at the National Center for Biotechnology Information (NCBI) website (www.ncbi.nlm.nih.gov) with the deduced protein sequences of DMAT synthase from Claviceps fusiformis (24) and LPS1 of $N$. lolii (16) or C. purpurea (26) as query sequences. The nature of genes linked to the ergot alkaloid biosynthesis genes or candidate genes was investigated by retrieving contiguous 2-kb segments of DNA from the A. fumigatus genome and querying the nonredundant protein database of NCBI with these sequences by application of the blastx search algorithm (1).

Knockout of dmaW in A. fumigatus. Fungal DNA for amplification by PCR was isolated following the Gene Clean Spin protocol (Bio 101, Vista, CA). An internal fragment of $A$. fumigatus $d m a W$ (encoding amino acids 136 to 352 of the predicted 497 amino acid product) was amplified by PCR from primers $d m a W$ forward (5'TTGATCTGGAGTGGTTCCGC-3') and $\mathrm{dmaW}$ reverse (5'CGTTCATGCCGAGGTTGTG-3'). A $50 \mu \mathrm{L}$ PCR reaction contained $50 \mathrm{mM} \mathrm{KCl,} 10$ $\mathrm{mM}$ Tris- $\mathrm{HCl}(\mathrm{pH} 9.0), 0.1 \%$ (v/v) Triton X-100, $1.5 \mathrm{mM} \mathrm{MgCl}_{2}, 200 \mu \mathrm{M}$ of each deoxyribonucleotide triphosphate, $1 \mu \mathrm{M}$ of each primer, and 2.5 units of Taq DNA polymerase (Promega, Madison, WI), which was added once the thermocycler reached 95 
${ }^{\circ} \mathrm{C}$ in the initial denaturing period. The reaction began with an initial denaturing time of 2 $\min 30 \mathrm{~s}$ at $95^{\circ} \mathrm{C}$, followed by 35 cycles of $1 \mathrm{~min}$ at $94^{\circ} \mathrm{C}, 1 \mathrm{~min}$ at $55^{\circ} \mathrm{C}$, and $1 \mathrm{~min}$ at $72{ }^{\circ} \mathrm{C}$. The 651-bp $d m a W$ PCR product was ligated into pCR2.1 (Invitrogen, Carlsbad, CA) based on T/A overhangs and transformed into chemically competent Escherichia coli cells. The resulting 4.5-kb gene knockout construct, pDMAT1, was linearized at a unique $P p u \mathrm{MI}$ site within the $d m a W$ coding sequences prior to transformation of $A$. fumigatus.

Protoplasts of A. fumigatus were prepared according to Murray et al. (11), except that $5 \mathrm{mg} / \mathrm{mL}$ of Driselase (InterSpex, Foster City, CA), $1.3 \mathrm{mg} / \mathrm{ml}$ Novozyme 234 (InterSpex) and $25 \mu \mathrm{g} / \mathrm{mL}$ chitinase (Sigma, St. Louis, MO) were used as lysing enzymes. Cotransformation of the protoplasts with PpuMI-linearized pDMAT1 and the hygromycin resistance-conferring plasmid pMOcosX (12)(linearized at a unique NotI site) was performed as previously described by Panaccione et al. (16), but with $4 \mu \mathrm{g}$ of each DNA in $10 \mu \mathrm{L}$ of water. Considering the relative differences in the lengths of the respective cotransformed molecules, the equal masses introduced corresponded to an $\sim 2$ fold molar excess of the gene knockout construct (pDMAT1, $4.5 \mathrm{~kb}$ ) relative to the selectable marker (pMOcosX, $8.8 \mathrm{~kb})$. The transformation mixture was divided into 6 aliquots and plated on regeneration medium containing $300 \mu \mathrm{g} / \mathrm{mL}$ hygromycin (Calbiochem, La Jolla, CA) as previously described (16). Transformation plates were incubated at $37^{\circ} \mathrm{C}$ for two days and hygromycin-resistant colonies were transferred onto PDA plates containing $400 \mu \mathrm{g} / \mathrm{mL}$ hygromycin.

Transformants were screened for homologous recombination of pDMAT1 with the native $d m a W$ gene by PCR (same conditions as above, except annealing temperature 
was $57^{\circ} \mathrm{C}$ ) from primers UF (5'-TGTAAAACGACGGCCAGTGAAT-3'; which anneals to vector sequences near the universal primer annealing site pCR2.1) and $3 \mathrm{~S}\left(5^{\prime}-\right.$ AAGTAAGTCCCGAGCTGTTCAT-3'; which anneals to $d m a W$ sequences near the 3'end of the gene and flanking the intended site of integration)(Fig. 1A). Transformants yielding a fragment of the expected size for a gene knockout at $d m a W(1.2 \mathrm{~kb})$ were purified to nuclear homogeneity by culturing from individual, germinated conidia. Single-spored colonies were again screened by PCR (conditions as described immediately above but with annealing temperature of $\left.60^{\circ} \mathrm{C}\right)$ but with primers UR $\left(5^{\prime}-\right.$ AGCTATGACCATGATTACGCCA-3'), which anneals to vector sequences near the reverse primer annealing site of $\mathrm{pCR} 2.1$, and primer $5 \mathrm{~S}$ (5'TAGTGAGATACACATTCGAGCC-3'), which anneals to $d m a W$ sequences near the 5'end of the gene and flanking the intended site of integration (Fig. 1A). In this way, both the 5' and 3' borders of the integration site were analyzed by PCR. Candidate gene knockout strains also were screened with primers $5 \mathrm{~S}$ and $3 \mathrm{~S}$ under PCR conditions described above and with an annealing temperature of $57^{\circ} \mathrm{C}$. Finally, transformants producing fragments of expected sizes in the PCR analyses were analyzed further by Southern hybridization as described below.

Fungal DNA for Southern blot hybridization was digested with SalI, sizefractionated by electrophoresis through $0.8 \%$ agarose, and transferred to Biodyne A nylon membrane (Pall-Gelman Sciences, Ann Arbor, MI) by capillary action with 20× $\mathrm{SSC}(1 \times \mathrm{SSC}$ is $150 \mathrm{mM} \mathrm{NaCl}+15 \mathrm{mM}$ trisodium citrate) as the transfer medium. A dmaW probe was labeled with DIG-dUTP by amplifying wild type genomic DNA by PCR from primers $d m a W$ forward and $d m a W$ reverse (described above). PCR conditions 
were as described above except with 1× DIG DNA Labeling Mix (Roche Applied Science, Indianapolis, IN) replacing standard dNTPs. Hybridization was performed at 65 ${ }^{\circ} \mathrm{C}$ in $5 \times \mathrm{SSC}, 0.1 \%(\mathrm{w} / \mathrm{v}) \mathrm{N}$-lauroylsarcosine, $0.2 \%(\mathrm{w} / \mathrm{v})$ sodium dodecyl sulfate, $1 \%$ (w/v) blocking reagent (Roche), $10 \mathrm{mM}$ maleic acid, and $15 \mathrm{mM} \mathrm{NaCl}(\mathrm{pH}$ 7.5). Final washes were with $0.5 \times \mathrm{SSC}+0.1 \% \mathrm{SDS}$ at $65^{\circ} \mathrm{C}$. Bound probe was detected with antiDIG antibody followed by chemiluminescence by standard methodology (DIG Manual, Roche Applied Science, Indianapolis, IN).

An ergot alkaloid-deficient $d m a W$ knockout strain was complemented with a fulllength $d m a W$-containing fragment produced by PCR from primers 5'GAGAGTGGTGTTGAGAGCTGCC-3' and 5'-CCTTCAGGCTTCATGAACGGAC-3', which prime amplification of a 3.4-kb fragment containing the entire $\mathrm{dmaW}$ coding sequence along with 784 bp of 5'-flanking sequence and 1,123 bp of 3'-flanking sequence. PCR conditions were as described above except the annealing temperature was $57^{\circ} \mathrm{C}$ and the extension time at $72{ }^{\circ} \mathrm{C}$ was 3 min $20 \mathrm{~s}$. The PCR product was cotransformed into a $d m a W$ knockout strain along with phleomycin resistance-conferring plasmid pBC-phleo (22; Fungal Genetics Stock Center, University of Missouri-Kansas City, Kansas City, MO). Selection was on complete regeneration medium (16) containing phleomycin at $100 \mu \mathrm{g} / \mathrm{mL}$.

Analysis of ergot alkaloids. Ergot alkaloids were analyzed from conidia produced on PDA by extraction with $80 \%$ methanol and analyzed by HPLC with fluorescence detection as described previously (13). 


\section{RESULTS}

Identification and analysis of a dmaW homolog in A. fumigatus. A search of the $A$. fumigatus genome database revealed an excellent match for C. fusiformis dmaW, which encodes the enzyme dimethylallyltryptophan synthase (DMAT synthase) that catalyzes the first step in the ergot alkaloid pathway in clavicipitaceous fungi. The deduced $A$. fumigatus DMAT synthase was 50\% identical to DMAT synthase from C. fusiformis (24), 53\% identical to DMAT synthase from C. purpurea (26), and 59\% identical to the DMAT synthase of Neotyphodium sp. Lp1 (29). The A. fumigatus dmaW sequence contained a single intron at an identical position to the single intron in the $d m a W$ genes isolated from the Claviceps and Neotyphodium spp. (data not shown).

A gene knockout construct, designed to integrate at $d m a W$ by a single crossover (Fig. 1) was targeted to the $d m a W$ locus in 3 of 25 hygromycin-resistant transformants analyzed (Figs. 1 and 2). PCR analysis of the 5' and 3 ' junctions of the native $d m a W$ gene and the integrated gene knockout construct yielded fragments of the expected length for homologous recombination of such a construct at $d m a W$ (Figs. 1B and 1C). PCR amplification of the gene knockout strains from primers $5 \mathrm{~S}$ and $3 \mathrm{~S}$, spanning the site of recombination, failed to yield the $1.2-\mathrm{kb}$ product amplified from the wild-type isolate (data not shown). Southern hybridization of DNA from the putative knockout strains digested with SalI, which has sites flanking the targeted integration site and not within the knockout construct, indicated that three or more copies of the knockout construct integrated in tandem at $d m a W$ in these transformants (Fig. 2).

The dmaW knockout strains did not produce any of the known ergot alkaloids (Fig. 3). Additional peaks, including uncharacterized molecules eluting at $55.5 \mathrm{~min}$ and 
56 min (Fig. 3) and a minor peak eluting at 32.8 min (evident only upon viewing the traces at much higher sensitivity), also were eliminated by knockout of $d m a W$. The peak eluting at 32.8 minutes is proposed to contain one or more of the isomers of chanoclavine based on a measured mass of 256 and its co-elution with a chanoclavine standard. The later-eluting molecules have not been characterized further.

Transformation of strain $d m a W$ ko6 with a DNA fragment containing the entire dmaW coding sequences plus $784 \mathrm{bp}$ of 5'-flanking sequences and 1,123 bp of 3' flanking sequences restored the ability to produce ergot alkaloids (Fig. 3). The complementing fragment was determined to have integrated ectopically, because the gene knockout construct was retained at the native $d m a W$ locus (data not shown). All known $A$. fumigatus ergot alkaloids were produced in the complemented strains (Fig. 3).

Genes clustered with dmaW in A. fumigatus. Database searches with five $C$. purpurea gene sequences, which in addition to $d m a W$ have been proposed to be involved in ergot alkaloid biosynthesis (but which have not yet been functionally analyzed), identified potential homologs clustered with each other and with the dmaW homolog on A. fumigatus (Fig. 4). These included two putative oxidoreductase-encoding genes identified as cpox 1 and cpox2, as well as a catalase-encoding gene, and two unidentified open reading frames (labeled $\operatorname{orf} A \mathrm{Cp}$ and $\operatorname{orf} B \mathrm{Cp}$ ) whose sequence and close linkage with $d m a W$ are conserved in C. purpurea $(25,26)$ and C. fusiformis (10). Despite the similarity in the composition of the clusters of genes associated with $d m a W$ in $A$. fumigatus and C. purpurea, the physical arrangement in terms of the orientation and order of genes within the cluster differed in the two fungi (Fig. 4). 
Analysis of additional sequences clustered on the $d m a W$ contig of $A$. fumigatus identified several other genes that may encode enzymes in the ergot alkaloid pathway (Fig. 4). These include sequences similar to cytochrome P450 monooxygenases, a FADdependent monooxygenase, and short chain alcohol dehydrogenases. Additional sequences whose deduced functions are consistent with roles in formation of the side chains that vary among the three fumigaclavines also were identified. These include any of several monooxygenases or hydroxylases, an O-acetyl transferase, and a divergent $d m a W$-like (prenyl transferase) gene. The predicted product of the divergent $d m a W$-like gene had 25\% identity with the deduced product of $A$. fumigatus dmaW required for ergot alkaloid biosynthesis and had 26\%,25\%, and 27\% identity with the deduced products of dmaW from C. purpurea, C. fusiformis, and Neotyphodium sp. Lp1, respectively.

\section{DISCUSSION}

Our results demonstrate that the genome of the A. fumigatus contains a homolog of dmaW that is required for ergot alkaloid biosynthesis. The loss of all known ergot alkaloids upon inactivation of $d m a W$ in $A$. fumigatus indicates that this gene controls the determinant step in the ergot alkaloid pathway of A. fumigatus, as it does in the clavicipitaceous fungus Neotyphodium sp. Lp1 (29). Moreover, in the A. fumigatus genome, dmaW is clustered with genes similar to, and extending, the ergot alkaloid gene cluster observed in the ergot fungus C. purpurea. Collectively the data indicate a common biosynthetic and genetic origin for the ergot alkaloids of A. fumigatus with those of the ergot alkaloid-producing members of the Clavicipitaceae.

Each of the three gene knockout strains investigated contained three or more copies of the knockout construct integrated at $d m a W$. The multiple integrations may have 
resulted from either or both of two modifications of the more typical gene knockout strategy that we applied in this experiment. First, the knockout construct was introduced by cotransformation at two-times the molar concentration of the selectable marker construct. Second, the knockout was created by integrating an internal fragment of the gene via a single crossover, a strategy that has resulted in multiple integrations in previous studies with other fungi (e.g., 2, 17, 18,21). This particular approach was chosen for two reasons. Knockout construct can be easily prepared in a single cloning step, which should facilitate similar analyses on many of the genes in the cluster. Also, since we intended to complement the mutation in a subsequent step, any phenotype associated with ectopic integration of the cotransformed selectable marker construct would have been revealed at that stage. The observations that three independent transformants containing the integration at $d m a W$ lacked ergot alkaloids and the complementation restored ergot alkaloid production indicate that the strategy provides a reasonable means for rapid analysis of gene function.

Aspergillus fumigatus produces at least four ergot alkaloids, all belonging to the clavine class of ergot alkaloids $(4,13,23)$. Close analysis of peaks eliminated in the gene knockout versus wild-type strains in this current study indicated that conidia of $A$. fumigatus also contain small quantities of chanoclavine. This is not surprising since chanoclavine is an early pathway intermediate in the biosynthesis of all known ergot alkaloids and it accumulates to varying degrees in different ergot alkaloid producers $(7,8$, $14,15,25)$.

Among the ergot alkaloids produced by A. fumigatus, only festuclavine and chanoclavine have been detected in any of the studied clavicipitaceous ergot alkaloid 
producers $(6,7,15,19)$. Conversely, the ergot alkaloid-producing members of the Clavicipitaceae produce numerous ergot alkaloids that are not found in A. fumigatus. These include the ergopeptines (tripeptide derivatives of lysergic acid) and simple amides of lysergic acid (such as ergonovine and ergine). The production of both the ergopeptine and simple amide classes of ergot alkaloids is dependent upon the activities or products of lysergyl peptide synthetases $(5,15,16)$. Based on the ergot alkaloid profiles and sequences clustered with $d m a W$ in $A$. fumigatus versus $C$. purpurea, we hypothesize that the early stages of the pathway are shared in the A. fumigatus and C. purpurea but that later steps in the pathways differ in the respective fungi.

The ergot alkaloid pathways of A. fumigatus and C. purpurea probably share several steps in addition to the determinant step catalyzed by DMAT synthase. The cluster of genes immediately around $d m a W$ in A. fumigatus contains five hypothetical genes that are highly similar to genes previously hypothesized to be involved in the early stages of the ergot alkaloid pathway of $C$. purpurea $(5,25,26)$. These include the following: a sequence labeled $\operatorname{orf} B$, which encodes a product similar to a methyl transferase, an activity required for the second step in the pathway; two genes (cpoxl and cpox2) encoding oxidoreductase that may catalyze early steps in the pathway $(7,14,25)$; a sequence labeled $\operatorname{orf} A$, for which a biochemical function has not been proposed; and, a catalase-encoding gene. A function for the catalase in ergot alkaloid biosynthesis is not obvious. One possibility is that it may act peroxidatively to oxidize the primary alcohol of chanoclavine to the corresponding aldehyde, which is a step required prior to cyclization of the fourth ring of the basic four-membered ergoline ring system. Interestingly, the orientation and position of three members of this putative core set of 
genes differ in the clusters of the two fungi, suggesting that numerous recombination events occurred after divergence of the lineages of these two fungi. Alternatively, if the cluster had been acquired by horizontal gene transfer, then the dissimilarities indicate than any such transfer would have been ancient.

We propose that after a shared early stage, the ergot alkaloid pathways of $A$. fumigatus and the clavicipitaceous fungi diverge to yield different end products. Cultures of A. fumigatus do not contain detectable levels of the later products of the pathway such as lysergic acid or lysergyl derivatives such as ergopeptines (13). Consistent with this hypothesis, searches with fragments of genes encoding LPS1 (an enzyme required for the assembly of the ergopeptine class of ergot alkaloids from lysergic acid and three amino acids) from N. lolii (16) or C. purpurea (26) produced only low-level matches to miscellaneous peptide synthetase genes in the A. fumigatus genome. The degree of identity observed was typical of the identity among peptide synthetases catalyzing the assembly of unrelated nonribosomally synthesized peptides in fungi (27), and none of these low-level matches was located near the $d m a W$ gene.

Conversely, the A. fumigatus cluster contained genes not found in C. purpurea cluster. Some of these hypothetical genes encode functions that we hypothesize to be involved in the later steps of the A. fumigatus pathway leading from festuclavine to the fumigaclavines, which are not produced by C. purpurea. For example, product of the gene in the A. fumigatus cluster predicted to encode an O-acetyltransferase may function in acetylating fumigaclavine $\mathrm{B}$ to fumigaclavine $\mathrm{A}$. The unusual, alternate $d \mathrm{ma}$ gene (labeled alt $d \mathrm{maW}$ in Fig. 4) may control the prenylation of fumigaclavine A to fumigaclavine $\mathrm{C}$. The lack of ergot alkaloids in the mutant containing a knockout at the 
typical $d m a W$ demonstrates that the highly divergent $d m a W$-like prenyl transferase gene found in the cluster is not functionally redundant with $\mathrm{dmaW}$ and, thus, may catalyze another prenylation step.

The available data for A. fumigatus extend further than the published ergot alkaloid cluster data from C. purpurea, revealing several additional genes potentially involved in ergot alkaloid production. Systematic gene knockouts and their characterization should allow definition of the early (and probably shared) steps of the ergot alkaloid pathway, several of which have been difficult to characterize biochemically $(7,8,14)$. Ergot alkaloid-deficient mutants such as the $d m a W$ knockout may be useful for assessing the contribution of ergot alkaloids to virulence towards animals or potential contributions of the alkaloids to the success of A. fumigatus.

\section{ACKNOWLEDGEMENTS}

This work was supported by USDA-NRI grant No. 2001-35319-10930 and Hatch funds. Sequencing of the A. fumigatus genome was funded by National Institute of Allergy and Infectious Disease grant U01 AI 48830 to David Denning and William Nierman, the Wellcome Trust, and Fondo de Investigaciones Sanitarias.

We thank Caroline Machado, Christopher Schardl, and Mark Farman (University of Kentucky) for access to data and helpful suggestions, and Brian Tapper (AgResearch Limited, New Zealand) and Miroslav Flieger (Czech Academy of Sciences) for the chanoclavine standard. Preliminary sequence data from the A. fumigatus genome were obtained from The Institute for Genomic Research at http://www.tigr.org. The assistance of Bill Nierman and Natalie Federova with questions concerning the data is greatly appreciated. 


\section{REFERENCES}

1. Altschul, S. F., T. L. Madden, A. A. Schaffer, J. Zhang, Z. Zhang, W. Miller, and D. J. Lipman. 1997. Gapped BLAST and PSI-BLAST: a new generation of protein database search programs. Nucleic Acids Res. 25:3389-3402.

2. Apel, P. A., D. G. Panaccione, F. R. Holden, and J. D. Walton. 1993. Cloning and targeted gene disruption of $X Y L 1$, a $\beta 1,4$-xylanase gene from the maize pathogen Cochliobolus carbonum. Mol. Plant-Microbe Interact. 6:467-473.

3. Clay, K., and C. Schardl. 2002. Evolutionary origins and ecological consequences of endophyte symbiosis with grasses. Am. Nat. 160:S199-S127.

4. Cole, R. J., J. W. Kirksey, J. W. Dorner, D. M. Wilson, J. C. Johnson Jr., A. N. Johnson, D. M. Bedell, J. P. Springer, K. K. Chexal, J. C. Clardy, and R. H. Cox. 1977. Mycotoxins produced by Aspergillus fumigatus species isolated from molded silage. J. Agric. Food Chem. 25:826-830.

5. Correia, T., Grammel, N., Ortel, I., Keller, U. and Tudzynski, P. 2003. Molecular cloning and analysis of the ergopeptine assembly system in the ergot fungus Claviceps purpurea. Chem. Biol. 10:1281-1292.

6. Flieger, M., M. Wurst, and R. Shelby. 1997. Ergot alkaloids — sources, structures, and analytical methods. Folia Microbiol. 42:3-30.

7. Floss, H. G. 1976. Biosynthesis of ergot alkaloids and related compounds. Tetrahedron 32:873-912.

8. Gröger, D., and H. G. Floss. 1998. Biochemistry of ergot alkaloids - achievements and challenges. Alkaloids 50:171-218. 
9. Keller, N. P., and T. M. Hohn. 1997. Metabolic pathway gene clusters in filamentous fungi. Fungal Genet. Biol. 21:17-29.

10. Machado, C. 2004. Studies of ergot alkaloid biosynthesis genes in clavicipitaceous fungi. Ph.D. Thesis. University of Kentucky, Lexington.

11. Murray, F. R., G. C. M. Latch, and D. B. Scott. 1992. Surrogate transformation of perennial ryegrass, Lolium perenne, using genetically modified Acremonium endophyte. Mol. Gen. Genet. 233:1-9.

12. Orbach, M. J. 1994. A cosmid with a $\mathrm{Hy}^{\mathrm{R}}$ marker for fungal library construction and screening. Gene 150:159-162.

13. Panaccione, D. G., and C. M. Coyle. 2005. Abundant respirable ergot alkaloids from the common airborne fungus Aspergillus fumigatus. Appl. Environ. Microbiol. $71: 3106-3111$.

14. Panaccione, D. G., and C. L. Schardl. 2003. Molecular genetics of ergot alkaloid biosynthesis, p. 399-424. In J. F. White Jr., C. W. Bacon, N. L. Hywel-Jones, and J. W. Spatafora (ed.), The Clavicipitalean Fungi: Evolutionary Biology, Chemistry, Biocontrol, and Cultural Impacts. Marcel-Dekker, New York.

15. Panaccione, D. G., B. A. Tapper, G. A. Lane, E. Davies, and K. Fraser. 2003. Biochemical outcome of blocking the ergot alkaloid pathway of a grass endophyte. J. Agric. Food Chem. 51:6429-6437.

\section{Panaccione, D. G., R. D. Johnson, J. Wang, C. A. Young, P. Damrongkool, B.} Scott, and C. L. Schardl. 2001. Elimination of ergovaline from a grassNeotyphodium endophyte symbiosis by genetic modification of the endophyte. Proc. Natl. Acad. Sci. USA 98:12820-12825. 
17. Panaccione, D. G., J. S. Scott-Craig, J.-A. Pocard, and J. D. Walton. 1992. A cyclic peptide synthetase gene required for pathogenicity of the fungus Cochliobolus carbonum on maize. Proc. Natl. Acad. Sci. USA 89:6590-6594

18. Panaccione, D. G., M. McKiernan, and R. M. Hanau. 1988. Colletotrichum graminicola transformed with homologous and heterologous benomyl resistance genes retains expected pathogenicity to corn. Mol. Plant-Microbe Interact. 1:113120.

19. Porter, J. K., C. W. Bacon, J. D. Robbins, and D. Betowski. 1981. Ergot alkaloid identification in Clavicipitaceae systemic fungi of pasture grasses. J. Agric. Food Chem. 29:653-657.

20. Riederer, B., Han, M., and Keller, U. 1996. D-Lysergyl peptide synthetase from the ergot fungus Claviceps purpurea. J. Biol. Chem. 271:27524-27530.

21. Scott-Craig, J. S., D. G. Panaccione, F. Cervone, and J. D. Walton. 1991. Endopolygalacturonase is not required for pathogenicity of Cochliobolus carbonum on maize. Plant Cell 2:1191-1200.

22. Silar, P. 1995. Two new easy to use vectors for transformation. Fungal Gen. Newsl. 42:73.

23. Spilsbury, J. F., and S. Wilkinson. 1961. The isolation of festuclavine and two new clavine alkaloids from Aspergillus fumigatus Fres. J. Chem. Soc. 5:2085-2091.

24. Tsai, H.-F., H. Wang, J. C. Gebler, C. D. Poulter, and C. L. Schardl. 1995. The Claviceps purpurea gene encoding dimethylallyltryptophan synthase, the committed step for ergot alkaloid biosynthesis. Biochem. Biophys. Res. Co. 216:119-125. 
25. Tudzynski, P., T. Correia, and U. Keller. 2001. Biotechnology and genetics of ergot alkaloids. Appl. Microbiol. Biotechnol. 57:593-605.

26. Tudzynski, P., K. Holter, T. Correia, C. Arntz, N. Grammel, and U. Keller. 1999. Evidence for an ergot alkaloid gene cluster in Claviceps purpurea. Mol. Gen. Genet. 261:133-141.

27. Walton, J. D., D. G. Panaccione, and H. Hallen. 2004. Peptide synthesis without ribosomes, p. 127-162. In J. Tkacz, and L. Lange (ed.), Advances in Fungal Biotechnology for Industry, Agriculture, and Medicine. Kluwer Academic, New York.

28. Walzel, B., Riederer, B., and Keller, U. 1997. Mechanism of alkaloid cyclopeptide synthesis in the ergot fungus Claviceps purpurea. Chem. Biol. 4:223-230.

29. Wang, J., C. Machado, D. G. Panaccione, H.-F. Tsai, and C. L. Schardl. 2004. The determinant step in ergot alkaloid biosynthesis by an endophyte of perennial ryegrass. Fungal Genet. Biol. 41:189-198. 


\section{FIGURE LEGENDS}

Figure 1: (A) Diagrammatic representation of homologous recombination at $d m a W$. In the wt diagram, $\mathrm{F}$ and $\mathrm{R}$ represent annealing sites for primers $\mathrm{dmaW}$ forward and $\mathrm{dmaW}$ reverse, respectively, which amplify an internal portion of $d m a W$. Gene knockout construct, pDMAT1, containing the internal fragment of $d m a W$, was linearized at a unique $P p u$ MI site internal to $d m a W$ prior to transformation. The diagram labeled ko represents the dmaW locus with three copies of pDMAT1 integrated in tandem, disrupting $d m a W$. $\mathrm{UF}=$ annealing site for primer derived from universal primer sequences in vector; $3 \mathrm{~S}=$ annealing site for primer flanking the $3^{\prime}$ border of the site of integration; $\mathrm{UR}=$ annealing site for primer derived from reverse primer sequences in vector; $5 \mathrm{~S}=$ annealing site for primer flanking the 5 ' border of the site of integration. (B) Primers UR and 5S amplified the expected 852-bp band from a strain carrying the disrupted dmaW allele but not from the wild-type isolate. (C) Primers UF and $3 \mathrm{~S}$ amplified the indicated $1.2-\mathrm{kb}$ product only from the strain with disrupted $d m a W$. Relative mobility of relevant fragments (sizes in $\mathrm{kb}$ ) of BstEII-digested bacteriophage lambda is indicated in each panel.

Figure 2: Southern blot of SalI-digested DNA from non-transformed A. fumigatus and three $d m a W$ knockout mutants probed with a $d m a W$ fragment derived from primers $d m a W$ forward (F) and $d m a W$ reverse (R). Relative intensity of hybridization reflects minor differences in loading of samples. Relative mobility of relevant fragments (sizes in $\mathrm{kb})$ of BstEII-digested bacteriophage lambda is indicated. 
Figure 3: HPLC traces of non-transformed A. fumigatus (wt), a dmaW knockout strain (dmaW ko6), and strain $d m a W$ ko6 complemented by ectopic integration of a DNA fragment containing the gene $(\mathrm{dmaW} c t)$. Insert (within wt panel) contains overlaid traces of non-transformed A. fumigatus (solid line; wt), strain dmaW ko6 (dotted line; ko), and the complemented strain (dashed line; ct), shown at higher sensitivity to demonstrate the presence or absence of fumigaclavine $\mathrm{B} . \mathrm{B}=$ fumigaclavine $\mathrm{B} ; \mathrm{F}=$ festuclavine; $\mathrm{A}=$ fumigaclavine $\mathrm{A} ; \mathrm{C}=$ fumigaclavine $\mathrm{C}$.

Figure 4: Map of the genes clustered with $d m a W$ of $A$. fumigatus compared to the cluster surrounding dmaW of $C$. purpurea (redrawn from ref. $\{25\}$ ). Arrows indicate the orientation of transcription. Abbreviations: Cp, C. purpurea; ox/red, oxidoreductase; cat, catalase; OAc trans, O-acetyl transferase; P450, cytochrome P450 monooxygenase; lovA $\mathrm{P} 450$, similar to $\mathrm{P} 450$ involved in lovastatin biosynthesis; $p d a \mathrm{P} 450$, similar to $\mathrm{P} 450$ involved in pisatin demethylase activity; DH, short chain alcohol dehydrogenase; alt, alternate; OYE, old yellow enzyme; hydrox, hydroxylase; monoox, monooxygenase; $p s$, peptide synthetase. To the right of $\operatorname{orfB} \mathrm{Cp}$ in A. fumigatus (as drawn in Fig. 4) is a metalloproteinase-encoding gene and a retrotransposon-rich region, after which the assembly ends. To the left of the illustrated A. fumigatus cluster are several genes encoding unknown hypothetical proteins, a few additional genes with deduced products also potentially involved with ergot alkaloid biosynthesis, as well as interspersed genes for which no role in ergot alkaloid biosynthesis can be deduced. Genes that are found in the same relative position and orientation in the two clusters are indicated by inclusion in a solid-lined box. Genes previously proposed to be involved in ergot alkaloid production 
that are present in both clusters but differ in their relative position and orientation are enclosed in dashed-line boxes. Genes for which a function has been demonstrated by gene knockout $(5,16,29)$ are indicated with an asterisk. The $A$. fumigatus sequences illustrated span from nucleotides 2,894,958 to 2,926,335 of assembly 92 (long arm of chromosome 2; formerly assembly 72) of the A. fumigatus genome. Identifiers for the $A$. fumigatus sequences (as presented from left to right) are m19874, m19875, m19876, m19877, m19878, m19880, m19881, m19882, m19883, m19884, m19885, m19886, m19888, and m19891, respectively. Data are available from The Institute for Genomic Research (www.tigr.org). Accession number for C. purpurea orfA $=$ AY836771, and for C. purpurea orfB $=\mathrm{AY} 836772$. Accession numbers for the remainder of the genes found in the C. purpurea cluster are reported in refs. 5, 25, and 26. 


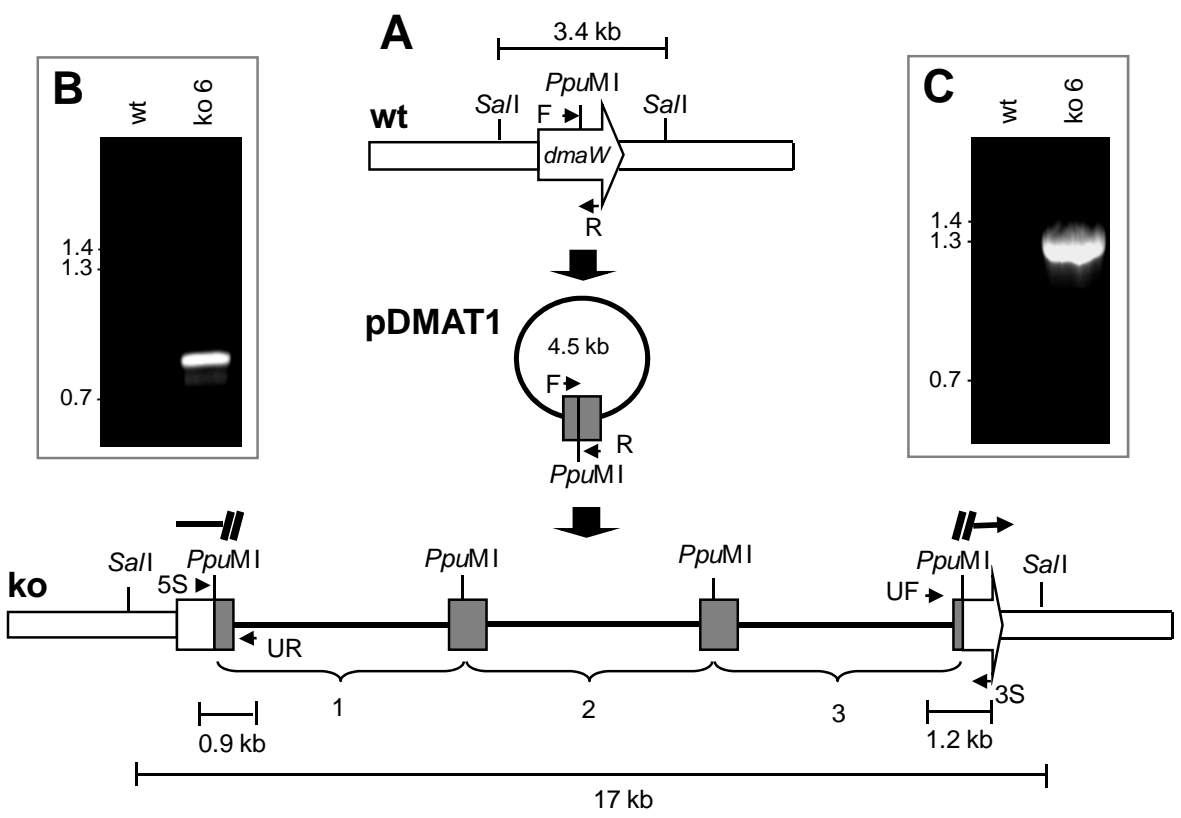

Coyle \& Panaccione, Fig. 1

Chapter 1 - Figure 1 


\begin{tabular}{|c|c|c|c|c|}
\hline & $\xi$ & $\begin{array}{l}0 \\
\stackrel{8}{y}\end{array}$ & $\hat{\grave{s}}$ & $\begin{array}{l}\Sigma \\
\stackrel{\Sigma}{Q}\end{array}$ \\
\hline $\begin{array}{r}14.1-1- \\
8.5-\end{array}$ & & $=$ & 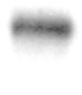 & $m$ \\
\hline 3.7 & 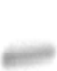 & & & \\
\hline 2.3 & & & & \\
\hline
\end{tabular}

Coyle \& Panaccione, Fig. 2

Chapter 1 - Figure 2 


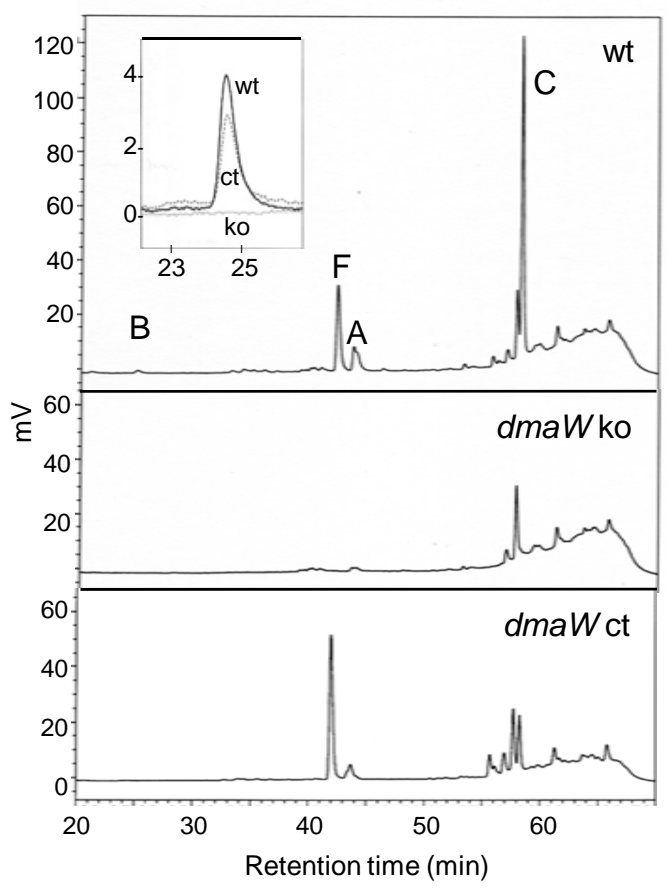

Coyle \& Panaccione, Fig. 3

Chapter 1 - Figure 3 


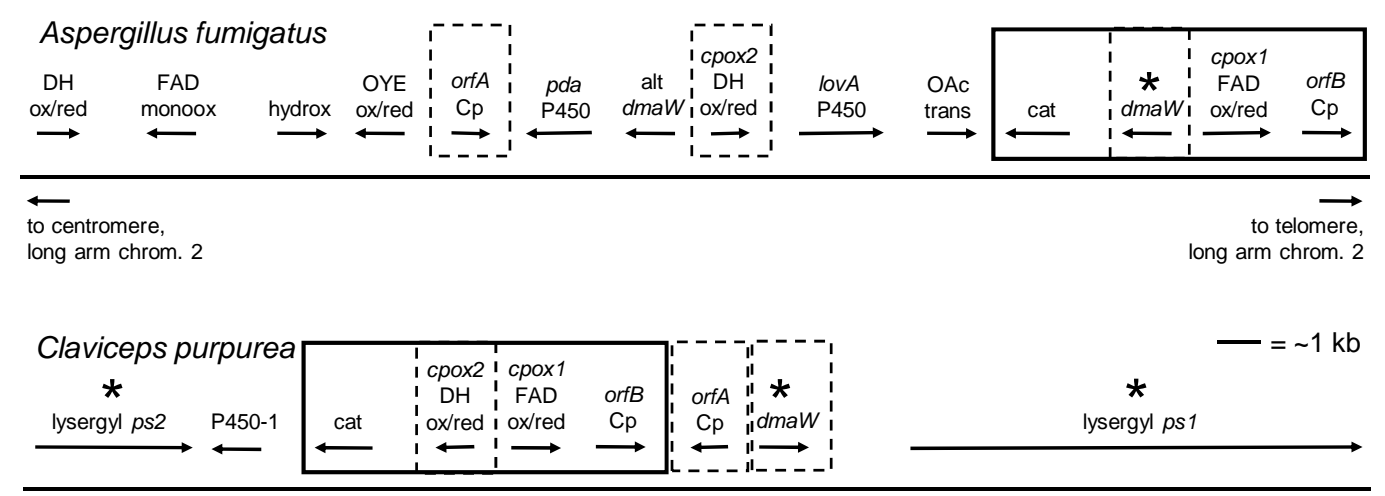

\section{Chapter 1 - Figure 4}




\title{
CHAPTER 2: Clustered genes, easE and easF, common to Aspergillus fumigatus and ergot fungi control early steps in ergot alkaloid biosynthesis
}

\author{
ABSTRACT \\ Ergot alkaloids are mycotoxins typically associated with clavicipitaceous fungi and cause \\ toxicoses to exposed individuals through their interactions with multiple neurotransmitter \\ receptors. Ergot alkaloids also are produced by the opportunistic human pathogen, \\ Aspergillus fumigatus; however, its chemical profile consists of certain clavines as \\ opposed to the lysergic acid derivatives typically observed in clavicipitaceous fungi. \\ These fungi are hypothesized to share early steps in ergot alkaloid synthesis prior to \\ divergence into two lineage-specific pathways. Genes involved in ergot alkaloid \\ synthesis are clustered, and several homologues are shared between the two groups of \\ fungi. This study focuses on two genes hypothesized to function early in ergot alkaloid \\ biosynthesis, easE and easF. Functional analysis via gene knockout demonstrated that \\ both genes are required for ergot alkaloid biosynthesis in A. fumigatus. When either of \\ the genes was knocked out, transformants failed to produce chanoclavine or any ergot \\ alkaloids downstream of chanoclavine, but different intermediates accumulated. Strains \\ with a mutated eas $F$ accumulated an alkaloid with properties of dimethylallyltryptophan \\ (DMAT), a result consistent with easF encoding the $\mathrm{N}$-methyl transferase that acts on \\ DMAT. Strains in which easE was mutated accumulated an intermediate with properties \\ of N-methyl-DMAT. These data indicate that easE encodes the first oxidase in the ergot \\ alkaloid pathway. Mutants of eas $F$ accumulated trace quantities of downstream \\ intermediates, suggesting a route to circumvent this enzyme, presumably by the activity
}


of DMAT synthase on abrine (N-methyl-transferase) which is present in cultures of $A$. fumigatus. This study further elucidates the early steps in the pathway in A.fumigatus, steps that are also shared with clavicipitaceous fungi.

Keywords: Aspergillus fumigatus, Claviceps spp., easE, easF, ergot alkaloid, methyltransferase, oxidoreductase

\section{INTRODUCTION}

Ergot alkaloids can be classified into three groups: clavines, ergopeptines, and simple amides $(12,14)$. Clavines are the simplest of the ergot alkaloids, some of which serve as precursors of lysergic acid. Ergopeptines and simple amides are derivatives of lysergic acid. Clavicipitaceous fungi, such as Claviceps spp. and Neotyphodium spp., produce different end products compared to the distantly related saprophyte Aspergillus fumigatus. All three types of alkaloids are found in clavicipitaceous species, but the spectrum of alkaloids can vary dependent on species and/or isolate $(3,6,9)$. Aspergillus fumigatus only produces clavines, such as festuclavine and fumigaclavines $\mathrm{A}, \mathrm{B}$, and $\mathrm{C}$ $(1,15,19)$. Panaccione (9) hypothesizes that the ergot alkaloid biosynthesis pathway diverges into two lineage-unique pathways after the production of chanoclavine aldehyde, resulting in different ergot alkaloid profiles between A. fumigatus and clavicipitaceous fungi.

Comparison of ergot alkaloid profiles and relevant DNA sequence data from different ergot alkaloid-producing fungi has led to the hypothesis that these fungi share early steps within the ergot alkaloid biosynthesis pathway $(2,9,12)$.

Dimethylallyltryptophan (DMAT) synthase is the determinant step in both A. fumigatus 
and Neotyphodium sp. Lp1 $(2,16,18)$. In A. fumigatus, C. purpurea, C. fusiformis, and E. festucae, the gene encoding DMAT synthase is clustered among several genes whose products have predicted functions that may play a role in the ergot alkaloid pathway. Seven genes are common to the DMAT synthase-based clusters of each of these fungi and have been labeled as eas genes (14). This study focuses on characterization of easE and eas $F$, two genes found in the A. fumigatus and the clavicipitaceous ergot alkaloid gene clusters, via gene disruption in A. fumigatus and biochemical analyses of the gene disruption mutants.

\section{MATERIALS AND METHODS}

Fungi and culture conditions. Aspergillus fumigatus isolate FGSC A1141 (10) and mutant derivatives were maintained on potato dextrose agar [PDA; dehydrated potato flakes (Pillsbury, Minneapolis, MN) (20 g/L), D-glucose (20 g/L), and agar (15 g/L)]. Protoplasts or DNA samples were prepared from cultures that were grown overnight in $100 \mathrm{~mL}$ of malt extract medium $(50 \mathrm{~g}$ malt extract, $50 \mathrm{~g}$ lactose, $5 \mathrm{~g}$ asparagine, and $0.4 \mathrm{~g}$ $\mathrm{K}_{2} \mathrm{HPO}_{4}$ per $\mathrm{L}$ ) in a $250 \mathrm{~mL}$ Erlenmeyer flask on an orbital shaker set at $37{ }^{\circ} \mathrm{C}$ and 150 rpm.

Disruption and complementation of easE and easF. Internal (incomplete) regions of each putative gene's coding sequence were amplified by PCR primed with the following oligonucleotides: easE-F (5'-CCAGATACATTGCCATCGCATG-3') and easE-R (5'-TGTTCCAACTGCTTGGCCAGAT-3') and easF-F (5'GCTTCAAAGATGGCAGCAGCTA-3') and easF-R (5'GAGTTGGCATAACTTAACGACG-3'). The $50 \mu \mathrm{L}$ PCR reaction contained $50 \mathrm{mM}$ $\mathrm{KCl}, 10 \mathrm{mM}$ Tris- $\mathrm{HCl}$ (pH 9.0), 0.1\% (v/v) Triton X-100, $1.5 \mathrm{mM} \mathrm{MgCl}_{2}, 200 \mu \mathrm{M}$ of 
each deoxyribonucleotide triphosphate, $1 \mu \mathrm{M}$ of each primer, and 2.5 units of Taq DNA polymerase (Promega, Madison, Wisconsin), which was added once the thermocycler reached $95^{\circ} \mathrm{C}$ in the initial denaturing period. The reaction began with an initial denaturing step of $2 \mathrm{~min}$ and $30 \mathrm{sec}$ at $95^{\circ} \mathrm{C}$, followed by 35 cycles of 1 min at $94{ }^{\circ} \mathrm{C}$ (denaturing), $1 \mathrm{~min}$ at $55^{\circ} \mathrm{C}$ (annealing), and $1 \mathrm{~min}$ at $72{ }^{\circ} \mathrm{C}$ (extension), with a final extension step of $72{ }^{\circ} \mathrm{C}$ for $7 \mathrm{~min}$. Amplification of the easE fragment was achieved with an annealing temperature of $57^{\circ} \mathrm{C}$. A disruption construct was generated to target each individual gene by ligating the PCR amplicon into the T/A overlap vector pCR2.1 (Invitrogen, Carlsbad, California). For easE and eas $F$, a 4.8-kb construct (pEASE1) and a $4.5-\mathrm{kb}$ construct (pEASF1) were produced, respectively (Fig. $1 \& 2$ ). Prior to transformation into A. fumigatus, each disruption construct was linearized at a unique restriction site located within the gene specific region of the construct. The restriction digests were as follows: pEASE1 with BspEI and pEASF1 with Bsu36I.

Preparation and transformation of the protoplasts were previously described by Murray et al. (7) with modifications noted by Coyle and Panaccione [(2); Chapter 2], and instead of separation of protoplasts from debris by step-gradient centrifugation, protoplasts were filtered through cheesecloth. In addition, the lysing enzymes were suspended in $0.7 \mathrm{M} \mathrm{NaCl}$ instead of osmotic medium to digest the fungal tissue. The filtrate was centrifuged ( $5 \mathrm{~min}, 3436 \mathrm{x}$ ) ) to pellet the protoplasts. After filtration, the protoplasts were washed twice in $0.7 \mathrm{M} \mathrm{NaCl}$ and once in STC $(1 \mathrm{M}$ sorbitol, $50 \mathrm{mM}$ Tris- $\mathrm{HCl} \mathrm{pH} \mathrm{7.4,} 50 \mathrm{mM} \mathrm{CaCl}_{2}$ (7) to remove digestive enzymes prior to final suspension in STC. Protoplasts were co-transformed with a linearized disruption construct corresponding to eas $E$ or $e a s F$ and a hygromycin resistance plasmid, pMOcosX (8), 
linearized at a unique NotI site. A preliminary high performance liquid chromatography (HPLC) screen of colonies that arose on transformation plates identified transformants with altered ergot alkaloid profiles. At least forty hygromycin-resistant colonies were isolated for each gene, and transformant colonies were screened in groups of ten until prospective mutants were identified. These colonies were then grown from single spores to achieve nuclear homogeneity and then analyzed for verification of homologous recombination (described below).

Homologous recombination between the disruption construct and its respective native gene was verified by three PCR assays similar to the PCR reaction described above, but with different primers and annealing temperatures. For easE, the $5^{\prime}$ border of the recombination event was confirmed by PCR (annealing temperature at $57^{\circ} \mathrm{C}$ ) primed with oligonucleotides UF (5'-TGTAAAACGACGGCCAGTGAAT-3', which anneals to vector sequences near the universal primer annealing site in pCR2.1) and easE-Fscrn (5'ACTGAAGACGAGACATGTCATG -3', complementary to easE sequences near the 5'end of the gene and flanking the intended site of integration) (Fig. 1A). The juxtaposition of sequences near the $3^{\prime}$ border of the integration event was verified by PCR (annealing temp $\left.57^{\circ} \mathrm{C}\right)$ primed from oligonucleotides UR (5'-AGCTATGACCATGATTACGCCA3', complementary to vector sequences near the reverse primer annealing site of pCR2.1), and easE-Rscrn (5'-TTCCTGTACGACACTCTGAAGT -3', which anneals to easE sequences near the 3'-end of the gene and flanking the intended site of integration) (Fig. 1A). PCR across the entire easE locus was conducted by priming with oligonucleotides easE-Fscrn and easE-Rscrn (both described above) at an annealing temperature of $57^{\circ} \mathrm{C}$. For eas $F$, the $5^{\prime}$ border of the recombination event was confirmed by PCR (annealing 
temperature at $48{ }^{\circ} \mathrm{C}$ ) primed with oligonucleotides UF (described above) and easF-Fscrn (5'-AAGTTGTCGAAGGTCTCACGAA -3', complementary to eas $F$ sequences near the 5 '-end of the gene and flanking the intended site of integration (Fig. 2A). The juxtaposition of sequences near the 3 ' border of the integration event was verified by PCR (annealing temp $48^{\circ} \mathrm{C}$ ) primed from oligonucleotides UR (described above) and easFRscrn (5'-GTGATTAGAGATGCTTCGTGC -3', which anneals to eas $F$ sequences near the 3 '-end of the gene and flanking the intended site of integration) (Fig. 2A). PCR across the entire eas $F$ locus was conducted by priming with oligonucleotides easF-Fscrn and easF-Rscrn (both described above) at an annealing temperature of $45^{\circ} \mathrm{C}$.

Integration of the transformation construct also was analyzed by Southern hybridization as described previously (2), except for substitution of the respective gene specific probe. Each gene-specific probe was made by amplifying wild-type A. fumigatus genomic DNA via PCR from primer pairs easE-F and easE-R or easF-F and easF-R, according to the PCR conditions described above, substituting $1 \times$ DIG DNA Labeling Mix (Roche, Mannheim, Germany) for unlabeled dNTPs. Knockout and wild-type genomic DNA were digested with ApaI for easE, and HincII for eas F for each corresponding Southern blot.

Each null mutant strain was complemented with a fragment containing a fulllength copy of the respective coding sequences along with $1.3-\mathrm{kb}$ and $1.4-\mathrm{kb}$ of 5 'flanking sequences and $0.8-\mathrm{kb}$ and $1.2-\mathrm{kb}$ of 3 '-flanking sequences for eas $F$ and easE, respectively. The complementing fragments were amplified by PCR using primer pairs described as follows: easE, WeasE-F (5'-CTGCTCCAGCAGGTAGATCTTGATTC -3') and WeasE-R (5'-TAGAGGAGCACCCAGCTGAATGTCCT -3') and eas F, WeasF-F 
(5'-TCCATTCTTCGCTCGTTCAACCAGCAGG -3') and WeasF-R (5'-

CAGGACCTGTACCTAAAGCCTGGTAACC -3'). These fragments were each amplified in a $50 \mu \mathrm{L}$ PCR reaction containing $15 \mathrm{mM}\left(\mathrm{NH}_{4}\right)_{2} \mathrm{SO}_{4}, 50 \mathrm{mM}$ Tris- $\mathrm{HCl}(\mathrm{pH}$ 9.0), $0.1 \%(\mathrm{v} / \mathrm{v})$ Triton X-100, $1.5 \mathrm{mM} \mathrm{MgCl}_{2}, 200 \mu \mathrm{M}$ of each $\mathrm{dNTP}, 1 \mu \mathrm{M}$ of each primer, and 1 unit of DyNAzyme DNA polymerase (New England Biolabs, Beverly, MA) added during the initial denaturing step. The thermocycler program was as described above. However, the annealing temperatures and extension periods differed from above and from each other and they are as follows: eas $E, 55^{\circ} \mathrm{C}$ and $4 \mathrm{~min} 30 \mathrm{sec}$; and easF, $65^{\circ} \mathrm{C}$ and $3 \mathrm{~min} 20 \mathrm{sec}$. Each PCR product was cotransformed into their respective gene's-disrupted mutant along with a phleomycin resistance plasmid, pBCphleo (Fungal Genetics Stock Center, University of Missouri-Kansas City, Kansas City, MO). Transformants were selected on complete regeneration medium (11) containing $100 \mu \mathrm{g} / \mathrm{mL}$ phleomycin and incubated at $37^{\circ} \mathrm{C}$. Complemented transformants were cultured from individual spores to achieve nuclear homogeneity. To test for the presence of the original disruption construct, PCR reactions were performed as described above (i.e. easE-F and UF). An additional PCR screen was performed on each complemented transformant to test for the presence of the introduced wild-type allele. These reactions were primed with primer pairs and reaction conditions stated above (i.e. WeasE-F and WeasE-R).

Analysis of Ergot Alkaloids. Cultures used for ergot alkaloid analysis were grown on PDA at $37^{\circ} \mathrm{C}$ for $1 \mathrm{wk}$. Samples were prepared from whole plate extractions in $4 \mathrm{~mL} \mathrm{100 \%} \mathrm{methanol,} \mathrm{vortexed,} \mathrm{and} \mathrm{then} \mathrm{rotated} \mathrm{end-over-end} \mathrm{for} \mathrm{at} \mathrm{least} 2 \mathrm{hr}$. Ergot alkaloids were detected and quantified from $20 \mu \mathrm{L}$ of the prepared sample by reverse- 
phase HPLC on a C18 column with fluorescence detection based on excitation and emission wavelengths of $272 \mathrm{~nm}$ and $372 \mathrm{~nm}$, respectively (10). The presence of ergot alkaloids that possess a 9,10 double bond was analyzed by passing eluates through a second fluorescence detector set with excitation and emission wavelengths of $310 \mathrm{~nm}$ and $410 \mathrm{~nm}$, respectively. The peaks corresponding to festuclavine and fumigaclavines A, B, and $\mathrm{C}$ were previously identified by mass spectral analyses (10) and confirmed by elimination of these peaks in mutants that lack a functional $d \mathrm{maW}$, the first gene in the ergot alkaloid pathway (2). The peak corresponding to chanoclavine was identified by coelution with authentic chanoclavine standard (provided by B.A. Tapper, AgResearch New Zealand).

Liquid surface cultures used for ergot alkaloid analysis were grown on malt extract medium at $37^{\circ} \mathrm{C}$ for 2 weeks, extracted in $4 \mathrm{~mL} 100 \%$ methanol over a period of $2 \mathrm{hr}$ with occasional vortexing. Extracts were centrifuged to pellet spores and other fungal debris and supernatants were concentrated in a speed vacuum and subjected to liquid chromatography - mass spectrometry (LC-MS) analysis (Chem. Dept., MIT). The LC-MS samples were resolved on an Acquity Ultra Performance BEH C18 column with a 1.7 micrometer particle size, $2.1 \times 100 \mathrm{~mm}$ dimension. LC-MS was performed at a constant flow rate of $0.5 \mathrm{~mL} / \mathrm{min}$ with a gradient described as follows: at 0 min: 20 parts acetonitrile and 80 parts $0.1 \%$ formic acid in water; at $5 \mathrm{~min}$ : 20 parts $0.1 \%$ formic acid in water and 80 parts acetonitrile; at $6 \mathrm{~min}: 80$ parts $0.1 \%$ formic acid in water and 20 parts acetonitrile. Masses were detected by a Micromass LCT Premier TOF MS with an electrospray ionization source. 


\section{RESULTS}

Screening by HPLC indicated altered ergot alkaloid profiles in multiple hygromycinresistant colonies for both easE and eas $F$ transformants. Incorporation of pEASE1 into the native easE gene in A. fumigatus via homologous recombination occurred in three of these transformants. For easF, one hygromycin-resistant colony was identified to contain the pEASF1 within the native easF gene.

DNA analyses via PCR and Southern blot confirmed insertion of the knockout (ko) constructs within their target gene. All mutant colonies were started from single spores to achieve homokaryotic cultures. For easE ko mutants, PCR screens across the 5' and 3' borders of the predicted integration site both produced the predicted $1.2-\mathrm{kb}$ product, whereas the wild type lacked amplification from this primer pair (Fig. 1B). A PCR screen designed to detect the intact wild-type locus was positive for the expected 1.3-kb PCR product in the wild-type strain but did not detect a wild-type allele in the eas $E$ ko, because insertion of the ko construct within the native locus produced a large distance between priming sites (Fig. 1B). PCR analysis of the integration site in an easF mutant produced 815-bp and 755-bp fragments from screens across the 5' and 3' borders of the integration site, as predicted (Fig. 2B). The screen to detect the wild-type locus did not render a product from null mutant strains, again due to the insertion of the ko construct within the native eas $F$ gene. The wild-type DNA did produce the 820 -bp product.

Southern blot analysis of easE mutants confirmed disruption of the native gene and showed multiple insertions of the pEASE1 within easE (Fig. 1C). The Southern blot analysis for eas $F$ clearly showed insertion of the knockout construct in the eas $F$ mutant 
due to a shift in the gene specific HincII fragment when compared to wild type (Fig. 2C). Multiple knockout constructs appeared to be inserted, which is not abnormal for this integration technique. The presence of several bands suggests additional integrations or recombination among tandemly inserted knockout contstructs. Complementation analyses (described below) show that the mutant phenotype was due to integration into easF.

HPLC analyses showed that mutants of either eas $F$ or easE did not produce chanoclavine, festuclavine, or fumigaclavines A, B, or C (Fig. 3A \& 4A). The mutants of eas $F$ accumulated a compound with a retention time of $43 \mathrm{~min}$ that was not observed in wild type or in the easE mutants when analyzed by HPLC (Fig. 3A). LC-MS analysis of this peak showed that the compound had a molecular ion with $\mathrm{m} / \mathrm{z}$ of 273.16 (Fig. 3B). Since fragmentation with this ESI-source instrument is rare, the $273.16 \mathrm{~m} / \mathrm{z}$ ion likely represents the molecular ion $\left[\mathrm{M}+\mathrm{H}^{+}\right]$of a compound with molecular weight of 272.16. The more sensitive analysis on the LC-MS indicated the presence of small quantities of downstream intermediates, such as festuclavine.

The easE ko accumulated a novel compound that eluted from the HPLC column at $44 \mathrm{~min}$ and by LC-MS yielded an ion with $\mathrm{m} / \mathrm{z}$ of 287.17 , indicative of the molecular ion of a compound of molecular weight 286.17 (Fig. 4). This mutant did not contain any other downstream intermediates.

Disrupted strains were complemented by cotransformation with a wild-type copy of their respective genes and NotI-linearized pBCphleo. A 4.6-kb fragment containing a functional copy of easE complemented the eas $E$ ko. Likewise, eas $F$ was complemented with a 2.9-kb PCR product containing a wild-type copy of the eas $F$ allele. Positive 
transformants were verified by PCR, when primed by either WeasE-f and WeasE-r or WeasF-f and WeasF-r (not shown). Amplification of the 5' and 3' regions flanking the site of integration of the original knockout construct confirmed that both easE and eas $F$ complemented strains were derivatives of their original knockout mutants. HPLC analyses demonstrated that colonies of the eas $E$ complemented and eas $F$ complemented strains accumulated festuclavine and fumigaclavines A, B, and C, similar to wild type (not shown).

\section{DISCUSSION}

These data demonstrate that eas $F$ and eas $E$ encode enzymes involved in the early steps of the ergot alkaloid pathway. Knockout mutants of easE and eas $F$ lacked normal ergot alkaloid accumulation in A. fumigatus, decreasing or eliminating production of festuclavine and fumigaclavine A, B and C. In addition, novel compounds with properties of intermediates in the ergot alkaloid pathway accumulated in these mutants, allowing proposed placement of the enzymes encoded by these genes at specific steps of the ergot alkaloid pathway.

A. fumigatus eas $F$ is located $257 \mathrm{bp}$ downstream of eas $E$ within the ergot alkaloid cluster on chromosome 2 and spans $1.1 \mathrm{~kb}$ of the genome [(2); Chapter 1]. Comparison of the linear sequence to a known protein database shows it has $56 \%$ and $60 \%$ identity to C. purpurea and N. lolii EasF, respectively. EasF, both in A. fumigatus and C. purpurea, has been hypothesized to encode a methyltransferase $(2,4,12)$, even though sequence data to support this were weak and closest sequence identity matches were to products of other unknown open reading frames. Knockout of eas $F$ resulted in accumulation of a compound with a molecular weight of 272.16, which is the molecular weight of DMAT, 
the first intermediate of the ergot alkaloid pathway. During the later stages of this work, Rigbers and Li (2008) published results of expression assays done in vitro in Escherichia coli. The expressed and isolated easF product methylated DMAT in the presence of $S$ adenosylmethionine, illustrating eas $F$ encodes for a methyltransferase capable of using DMAT as a substrate. My in vivo functional analysis of the eas $F$ gene supports the finding of the easF product functioning as a methyltransferase; the null mutant's inability to methylate DMAT resulted in accumulation of DMAT.

Interestingly, Unsold and Li (17) demonstrated that DMAT synthase of $A$. fumigatus, which typically uses tryptophan as a substrate, also can prenylate N-methyltryptophan (abrine) resulting in N-methyl-DMAT. This activity of DMAT synthase could theoretically bypass the requirement of the N-methyl transferase step. LC-MS analyses of my eas $F$ mutants indicated a degree of leakiness in that trace quantities of festuclavine and fumigaclavines $\mathrm{A}$ and $\mathrm{C}$ were detectable in selected ion traces (data not shown). Abrine was detectable in cultures of A. fumigatus, and thus may have served as an alternate substrate for DMAT synthase allowing a bypass of the mutated eas $F$ encoded step.

The gene, easE, is found on the $2^{\text {nd }}$ chromosome of A. fumigatus, between nucleotides 2925694-2927815; it is clustered with genes involved in ergot alkaloid production (2). The Aspergillus sequence is $45 \%$ similar to the C. purpurea easE protein sequence, $43 \%$ similar to $C$. fusiformis oxidase, and $47 \%$ similar to a putative oxidase of $N$. lolii. The eas $E$ sequence indicates that it encodes a FAD-containing oxidoreductase, a class of enzyme which would play a role in the proposed pathway $(9,12)$. My results show that strains in which eas $E$ is inactivated accumulate an intermediate with 
fluorescence properties typical of an ergot alkaloid with an 8,9 double bond and a molecular mass of 286.17, consistent with N-methyl-DMAT. Chanoclavine and alkaloids downstream of chanoclavine are all eliminated by knockout of easE. Based on these data, we hypothesize that the product of easE oxidizes N-methyl-DMAT. More specifically, the easE product likely hydroxylates the benzylic carbon (position 10) of the isoprene group of N-methyl-DMAT, an oxidation step proposed by Kozikowski et al. (5). Synthetic intermediates in which that particular carbon is oxidized are unstable; the 10hydroxylated N-methyl-DMAT spontaneously dehydrates to the corresponding diene (Fig 5), which has been demonstrated to be an authentic intermediate between N-methylDMAT and chanoclavine (5).

These findings suggest that the initial steps in the ergot alkaloid pathway proceed as follows: the determinant step, DMAT synthase (encoded by $d m a W$ ) (2), is followed first by a methyltransferase (eas $F$ ) and then by an oxidoreductase (easE). This study uncovers the early steps of the pathway, allowing for a better understanding of its dynamics both in A. fumigatus and clavicipitaceous fungi.

\section{ACKNOWLEDGEMENTS}

This project was supported by the National Research Initiative of the USDA CSREES, grants 2008-35318-04549 and 2005-35318-16184. I thank Johnathan Cheng and Dr. Sarah O'Connor (Massachusetts Institute of Technology) for performing mass spectrum analyses and for helpful suggestions on pathway intermediates. I also thank Chris Schardl (University of Kentucky) for helpful discussions, and Mike Hamstead and Dawn Chamberlain for their laboratory assistance. 


\section{REFERENCES}

1. Cole, R. J., J. W. Kirksey, J. W. Dorner, D. M. Wilson, J. C. Johnson, A. N. Johnson, D. M. Bedell, J. P. Springer, and K. K. Chexal. 1977. Mycotoxins produced by Aspergillus fumigatus species isolated from molded silage. J. Agric. Food Chem. 25:826-830.

2. Coyle, C. M., and D. G. Panaccione. 2005. An ergot alkaloid biosynthesis gene and clustered hypothetical genes from Aspergillus fumigatus. Appl. Environ. Microbiol. 71:3112-3118.

3. Flieger, M., M. Wurst, and R. Shelby. 1997. Ergot alkaloids -- sources, structures and analytical methods. Folia Microbiol. 42:3-30.

4. Haarmann, T., C. Machado, Y. Lübbe, T. Correia, C. L. Schardl, D. G. Panaccione, and P. Tudzynski. 2005. The ergot alkaloid gene cluster in Claviceps purpurea: extension of the cluster sequence and intra species evolution. Phytochemistry 66:1312-1320.

5. Kozikowski, A. P., C. Chen, J.-P. Wu, M. Shibuya, C.-G. Kim, and H. G.

Floss. 1993. Probing ergot alkaloid biosynthesis: Intermediates in the formation of ring C. J. Am. Chem. Soc. 115:2482-2488.

6. Kozlovsky, A. G. 1999. Producers of ergot alkaloids out of Claviceps genus, p. 479-499. In V. Kren and L. Cvak (ed.), In Ergot: The Genus Claviceps. Harwood Academic Publishers, The Netherlands.

7. Murray, F. R., G. C. M. Latch, and D. B. Scott. 1992. Surrogate transformation of perennial ryegrass, Lolium perenne, using genetically modified Acremonium endophyte. Mol. Gen. Genet. 233:1-9. 
8. Orbach, M. J. 1994. A cosmid with a HyR marker for fungal library construction and screening. Gene 150:159-162.

9. Panaccione, D. G. 2005. Origins and significance of ergot alkaloid diversity in fungi. FEMS Microbiol. Lett. 251:9-17.

10. Panaccione, D. G., and C. M. Coyle. 2005. Abundant respirable ergot alkaloids from the common airborne fungus Aspergillus fumigatus. Appl. Environ. Microbiol. 71:3106-3111.

11. Panaccione, D. G., R. D. Johnson, J. Wang, C. A. Young, P. Damrongkool, B. Scott, and C. L. Schardl. 2001. Elimination of ergovaline from a grassNeotyphodium endophyte symbiosis by genetic modification of the endophyte. Proc. Natl. Acad. Sci. USA 98:12820-12825.

12. Panaccione, D. G., C. Schardl, and C. M. Coyle. 2006. Pathways to diverse ergot alkaloid profiles in fungi, p. 23-52 In J. T. Romeo (ed.), Recent Advances in Phytochemistry, vol. 40. Elsevier, Amsterdam, The Netherlands.

13. Rigbers, O., and S.-M. Li. 2008. Ergot alkaloid biosynthesis in Aspergillus fumigatus: Overproduction and biochemical characterization of a 4dimethylallyltryptophan N-methyltransferase. J. Biol. Chem. 283:26859-26868.

14. Schardl, C., D. G. Panaccione, and P. Tudzynski. 2006. Ergot alkaloids-biology and molecular biology, p. 45-86. In G. A. Cordell (ed.), Alkaloids Chem. Biol., vol. 63. Academic Press, San Diego, CA.

15. Spilsbury, J. F., and S. Wilkinson. 1961. The isolation of festuclavine and two new clavine alkaloids from Aspergillus fumigatus Fres. J. Chem. Soc. 5:20852091. 
16. Tsai, H.-F., H. Wang, J. C. Gebler, C. D. Poulter, and C. L. Schardl. 1995. The Claviceps purpurea gene encoding dimethylallyltryptophan synthase, the committed step for ergot alkaloid biosynthesis. Biochem. Bioph. Res. Co. 216:119-125.

17. Unsöld, I. A., and S.-M. Li. 2005. Overproduction, purification and characterization of FgaPT2, a dimethylallyltryptophan synthase from Aspergillus fumigatus. Microbiology 151:1499-1505.

18. Wang, J., C. Machado, D. G. Panaccione, H.-F. Tsai, and C. L. Schardl. 2004. The determinant step in ergot alkaloid biosynthesis by an endophyte of perennial ryegrass. Fungal Genet. Biol. 41:189-198.

19. Yamano, T., K. Kishino, S. Yamantodani, and M. Abe. 1962. Investigation on ergot alkaloids found in cultures of Aspergillus fumigatus. Takeda Kenkyusho Nempo (Annual Report Takeda Research Laboratories) 21:95-101. 


\section{FIGURE LEGENDS}

Figure 1: Disruption strategy for easE. (A) EasE-F and easE-R primed amplification of internal region of easE. Gene disruption construct pEASE1 contains this internal fragment and was linearized at a unique $B s p E I$ site prior to transformation. The bottom panel illustrates insertion of three copies of the pEASE1 within genomic easE and the positions of primers used in PCR experiments to demonstrate integration at this locus. (B) Homologous recombination of the transforming DNA at easE was demonstrated by PCR with primers easE-Fscrn and UF confirming integration at the 5'-end of the gene and primers UR and easE-Rscrn confirming integration at the 3 '-end. Primer locations are shown in the bottom portion of part A. The wild-type (wt) screen, used to assess the presence of the wild-type allele, was a PCR primed from easE-Fscrn and easE-Rscrn, and amplification does not occur from DNA that has disruption constructs within the intended site of integration due to the increase in template length. PCR of non-transformed (wt) DNA gives a product of $1.3-\mathrm{kb}$. (C) Incorporation of transforming DNA at the targeted site was confirmed by Southern blot hybridization. Fungal DNA extracts were digested with $A p a \mathrm{I}$ and hybridized with a digoxigenin-dUTP labeled easE probe. The large size of the easE-containing fragments in strains easE ko1, easE ko15, and easE ko31 indicate three or more copies of pEASE1 are present in the integration site. The relative mobilities of relevant fragments of $B s t$ EII-digested bacteriophage lambda DNA are indicated on the left in panels B and C.

Figure 2: Disruption strategy for easF. (A) EasF-F and easF-R primed amplification of internal region of eas $F$. Gene disruption construct pEASF1 contains this internal 
fragment and was linearized at a unique Bsu36I site prior to transformation. The bottom panel illustrates insertion of three copies of the pEASF1 within genomic eas $F$ and the positions of primers used in PCR experiments to demonstrate integration at this locus. (B) Homologous recombination of the transforming DNA at eas $F$ was demonstrated by PCR with primers easF-Fscrn and UF confirming integration at the $5^{\prime}$-end of the gene and primers UR and easF-Rscrn confirming integration at the 3'-end. Primer locations are shown in the bottom portion of part A. The wild-type (wt) screen, used to assess the presence of the wild-type allele, was a PCR primed from easF-Fscrn and easF-Rscrn, and amplification does not occur from DNA that has disruption constructs within the intended site of integration due to the increase in template length. PCR of non-transformed (wt) DNA gives a product of 820-bp. (C) Incorporation of transforming DNA at the targeted site was confirmed by Southern blot hybridization. Fungal DNA extracts were digested with HincII and hybridized with a digoxigenin-dUTP labeled easF probe. The large sizes of the eas $F$-containing fragments in strain eas $F$ kol indicate three or more copies of pEASF1 are present in the integration site. Multiple bands suggest these strains are heterokaryons with varying number of pEASF1s inserted within target gene, due to deletion of one or more copies within some nuclei. The relative mobilities of relevant fragments of BstEII-digested bacteriophage lambda DNA are indicated on the left in panels B and C.

Figure 3: Chemical characterization of easF ko. (A) HPLC chromatogram of wildtype A. fumigatus (wt) and eas $F k o . \mathrm{B}=$ fumigaclavine $\mathrm{B} ; \mathrm{F}=$ festuclavine; $\mathrm{A}=$ fumigaclavine $\mathrm{A} ; \mathrm{C}=$ fumigaclavine $\mathrm{C} ; \mathrm{CH}=$ chanoclavine. Numbers next to peak are 
corresponding molecular weights. Excitation and emission wavelengths were $272 \mathrm{~nm}$ and $372 \mathrm{~nm}$, respectively. (B) The ESI-MS mass spectrum of easF ko contains a $273.16 \mathrm{~m} / \mathrm{z}$ ion, which corresponds to $\left[\mathrm{M}+\mathrm{H}^{+}\right]$for DMAT.

Figure 4: Chemical characterization of easE ko. (A) HPLC trace illustrating the chemical profiles of wild type (wt) and eas $E$ ko. $\mathrm{B}=$ fumigaclavine $\mathrm{B} ; \mathrm{F}=$ festuclavine; $\mathrm{A}=$ fumigaclavine $\mathrm{A} ; \mathrm{C}=$ fumigaclavine $\mathrm{C} ; \mathrm{CH}=$ chanoclavine. Numbers next to peak are corresponding molecular weights. Excitation and emission wavelengths were $272 \mathrm{~nm}$ and $372 \mathrm{~nm}$, respectively. (B) The ESI-MS mass spectrum of easE ko contains a 287.17 $\mathrm{m} / \mathrm{z}$ ion, which is characteristic of $\left[\mathrm{M}+\mathrm{H}^{+}\right]$for $\mathrm{N}$-methyl DMAT.

Figure 5: Early steps in ergot alkaloid biosynthesis. The gene, $d m a W$, has been shown to be the determinant step within the pathway and synthesizes DMAT (2). The data suggest the eas $F$ product $\mathrm{N}$-methylates DMAT, consistent with recent in vitro expression analyses (13). The oxidoreductase encoded by easE acts on N-methyl-DMAT, presumably resulting in the formation of the diene intermediate previously identified in precursor feeding studies (5). DMAPP = dimethylallylpyrophosphate; PPO = pyrophosphate Trp $=$ tryptophan; AdoMet $=$ S-adenosylmethionine; DMAT $=$ dimethylallyltryptophan. 
(a)

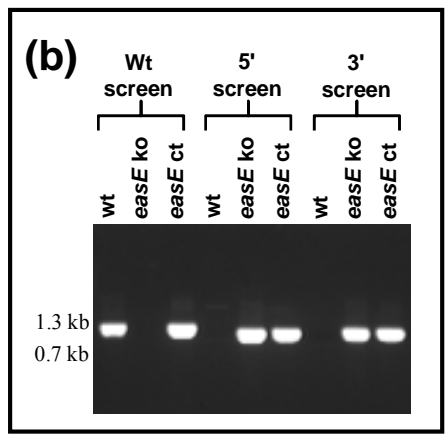

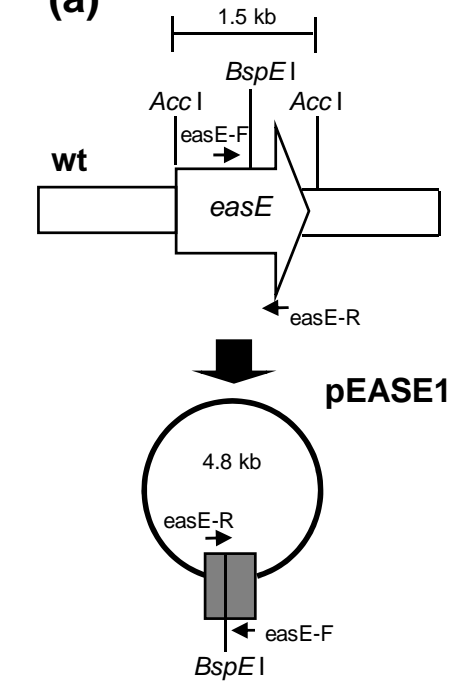

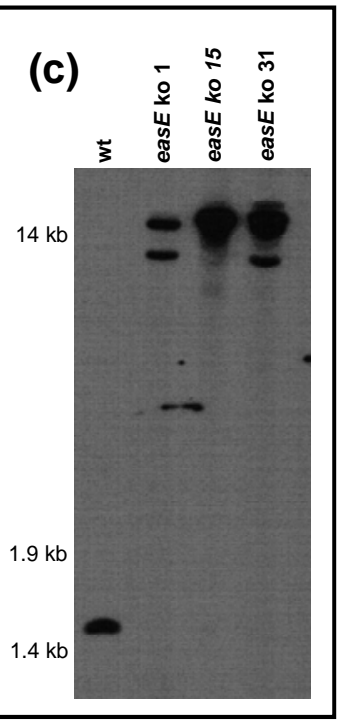

1
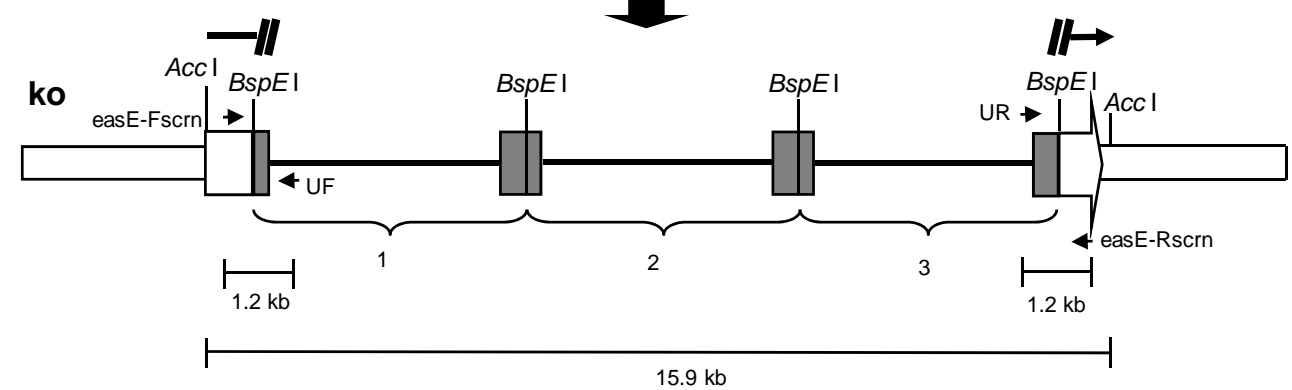

BspE I

Chapter 2 - Figure 1 
(a)

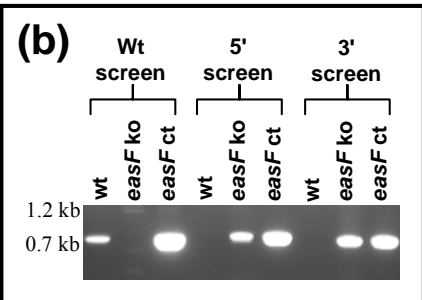
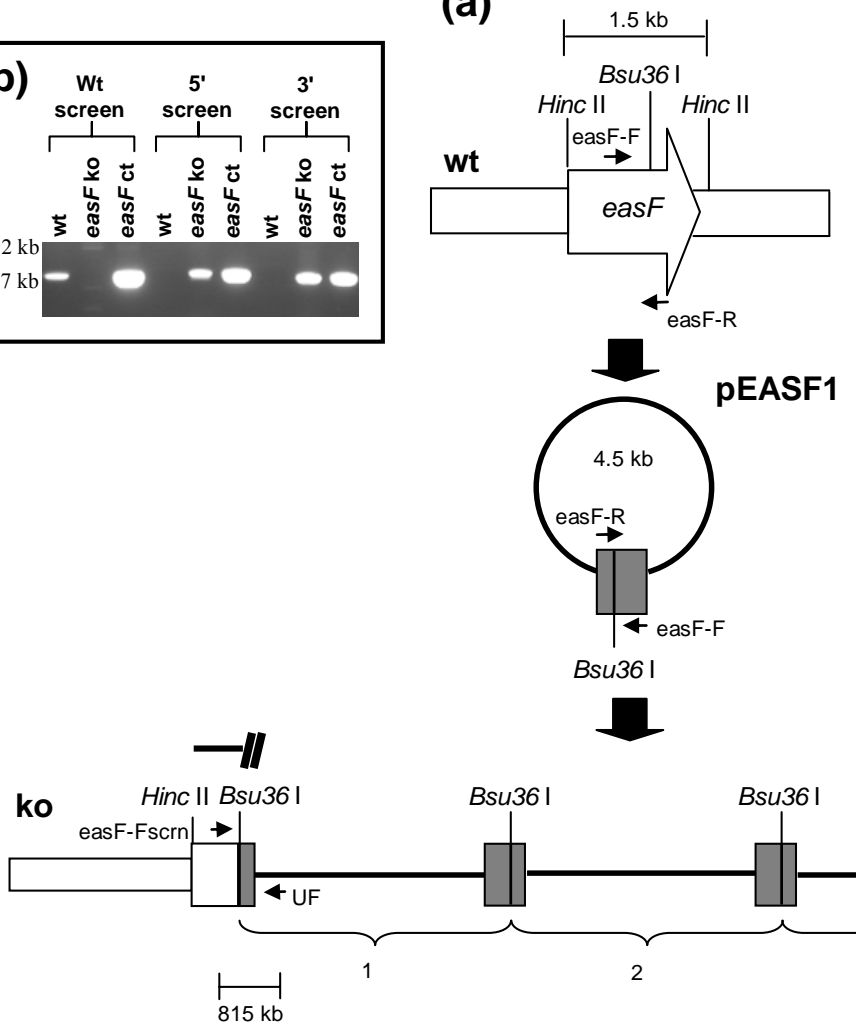

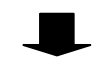

1
|| Bsu36 I

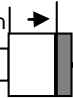

$815 \mathrm{k}$ $\longmapsto$
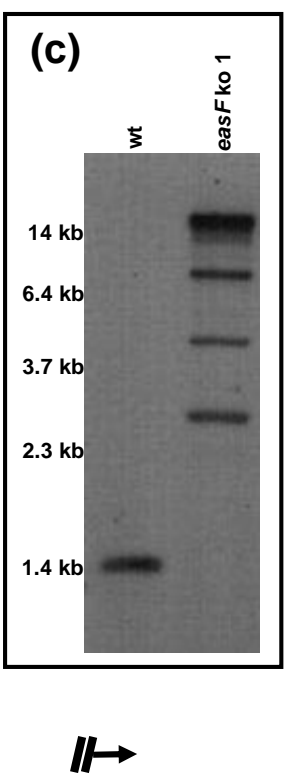

Bsu36 I Hinc II

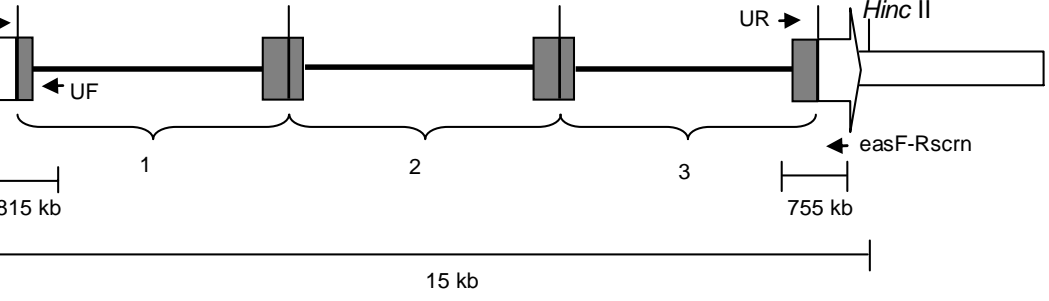

Chapter 2 - Figure 2 
A

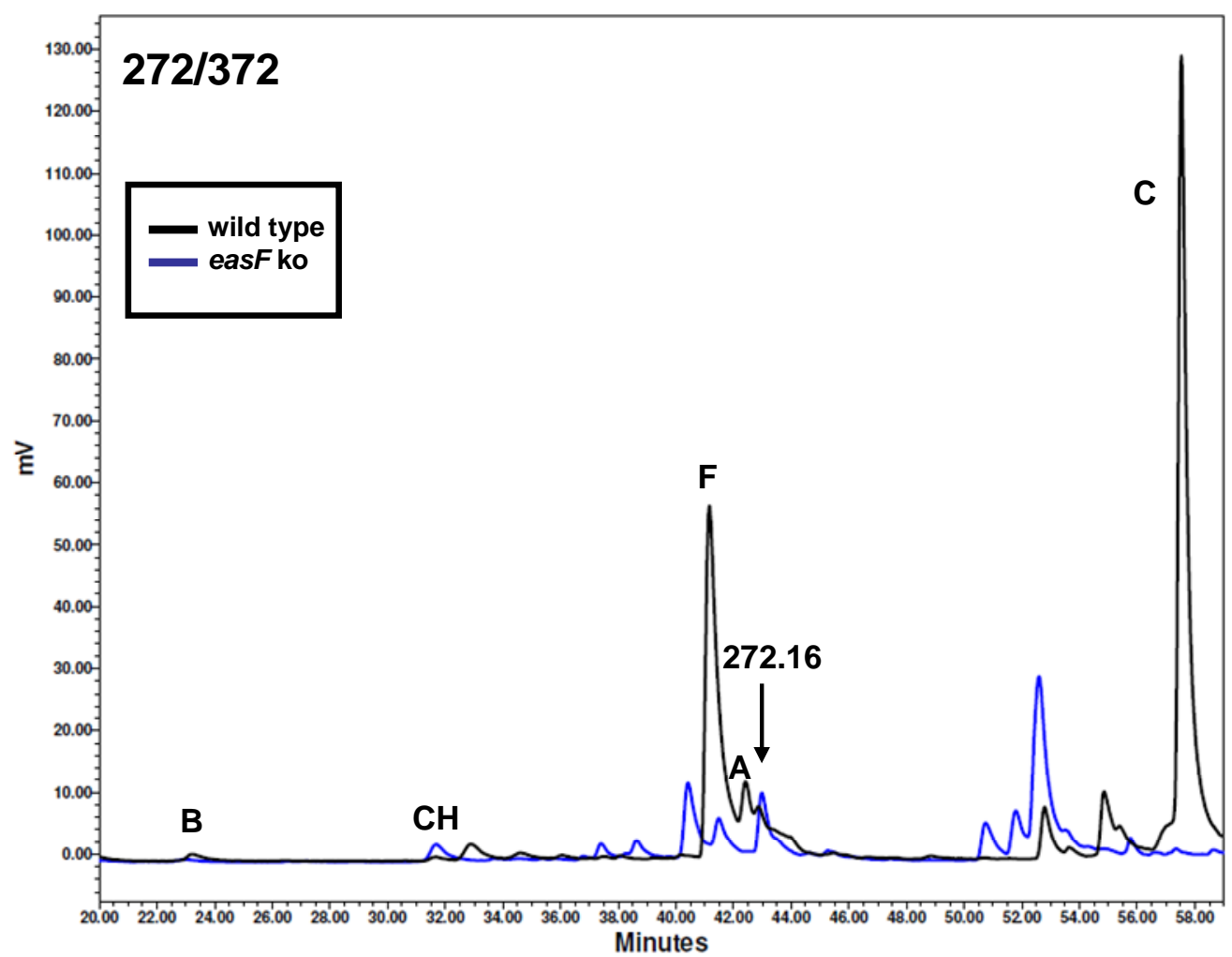

B

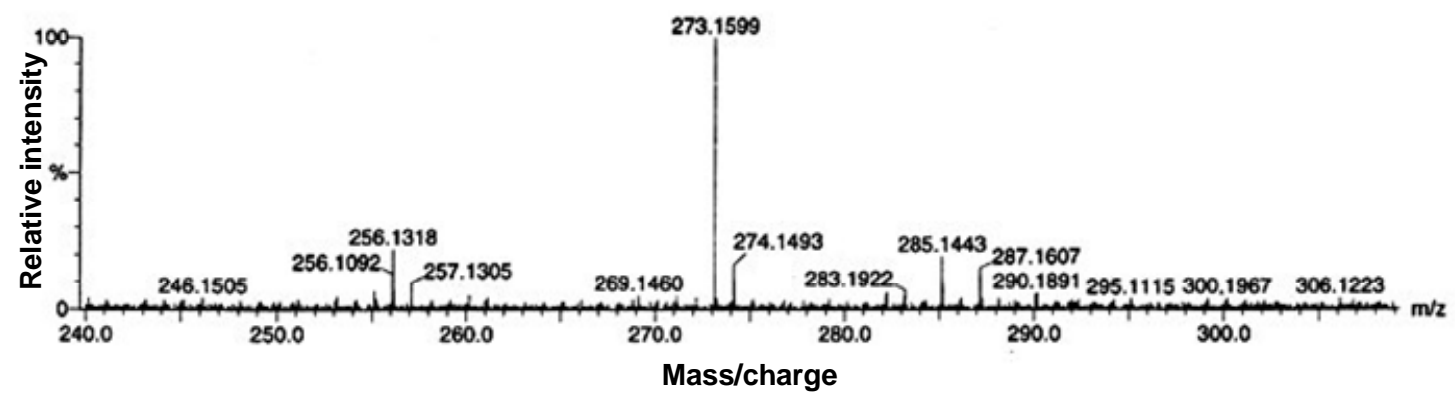

Chapter 2 - Figure 3 
A

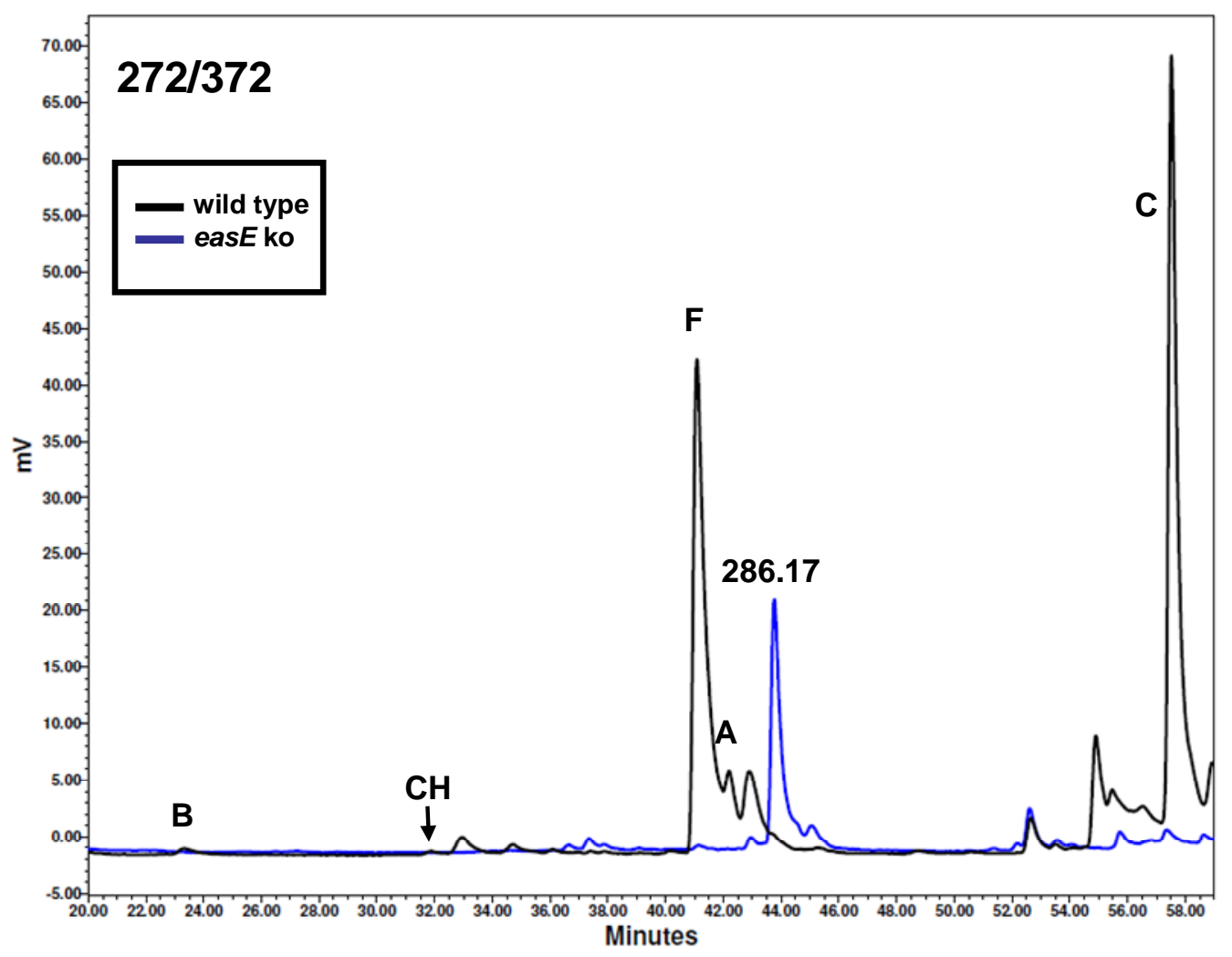

B

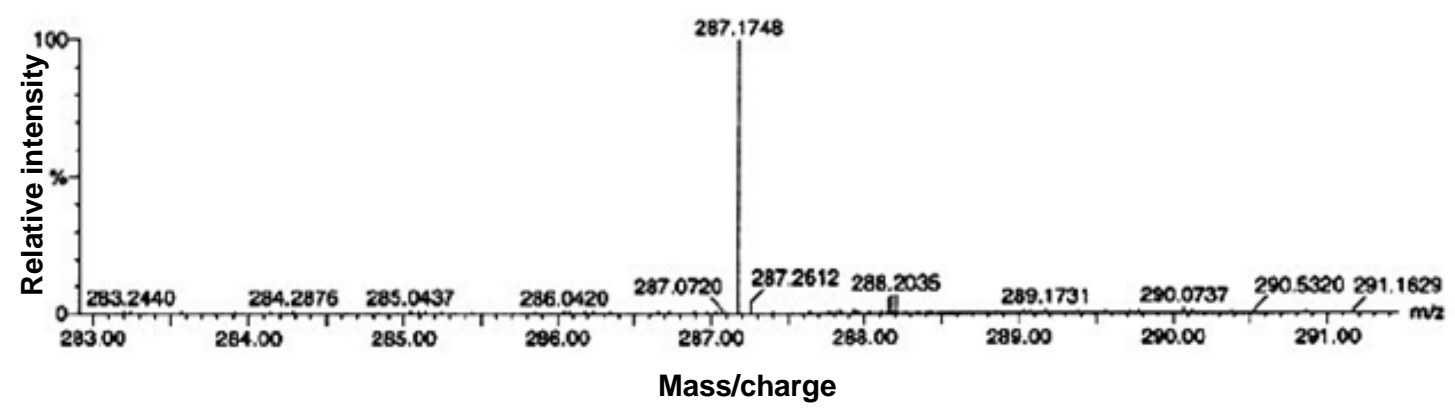

Chapter 2 - Figure 4 


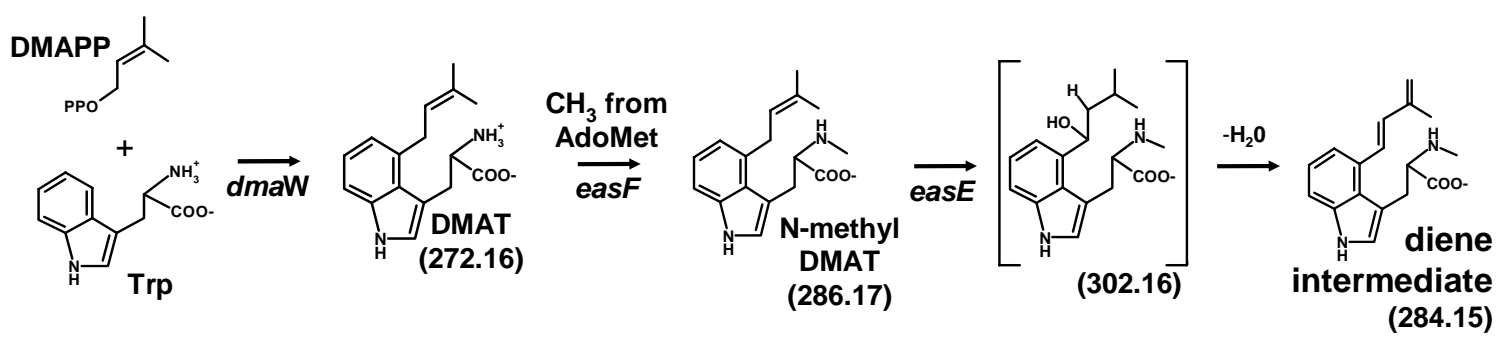

\section{Chapter 2 - Figure 5}




\title{
CHAPTER 3: An old yellow enzyme gene is required for ergot alkaloid biosynthesis and controls the branch point between Aspergillus fumigatus and Claviceps purpurea ergot alkaloid pathways
}

\author{
ABSTRACT \\ Ergot fungi in the genus Claviceps and several related grass endophytes in the genus \\ Neotyphodium produce toxic ergot alkaloids. Often these fungi produce a variety of ergot \\ alkaloids including clavines as well as lysergic acid derivatives. Ergot alkaloids also are \\ produced by the distantly related, opportunistic human pathogen, Aspergillus fumigatus. \\ However, this fungus produces festuclavine and fumigaclavines A, B, and C, which \\ collectively differ from clavines of clavicipitaceous fungi in saturation of the last \\ assembled of four rings in the tetracyclic ergoline structure. The two lineages are \\ hypothesized to share early steps of the ergot alkaloid pathway with divergence occurring \\ at some point after the synthesis of the tricyclic intermediate chanoclavine. Disruption of \\ eas $A$, a gene predicted to encode a flavin-dependent oxidoreductase of the old yellow \\ enzyme class, in $A$. fumigatus led to accumulation of chanoclavine and an intermediate \\ believed to be chanoclavine aldehyde. Complementation of the A. fumigatus eas A mutant \\ with a wild-type allele from the same fungus restored the wild-type profile of ergot \\ alkaloids. Complementation of the A. fumigatus eas $A$ knockout with a homologue of \\ eas $A$ from $C$. purpurea resulted in accumulation of alkaloids (agroclavine, setoclavine, \\ and its isomer isosetoclavine) typical of clavicipitaceous fungi. These data indicate that \\ the old yellow enzyme encoded by eas $A$ is required for ergot alkaloid biosynthesis and \\ functional differences in this enzyme in A. fumigatus and C. purpurea result in
}


divergence of their respective ergot alkaloid pathways. Sequence analysis of eas $A$ homologues from several fungi producing different classes of ergot alkaloids indicates a difference in a residue previously shown to be critical for double bond reduction in other old yellow enzymes.

Keywords: Ergot alkaloid, Aspergillus fumigatus, easA, Claviceps spp., old yellow enzyme (OYE)

\section{INTRODUCTION}

Different classes of ergot alkaloids are produced by members of two distinct fungal lineages. Clavicipitaceous species, which includes Claviceps spp. and Neotyphodium spp. (and its closely sexual relative, Epichloë spp.), are in the order Hypocreales and are known to synthesize lysergic acid derivatives, which include simple amides of lysergic acid and the more complex ergopeptines. These alkaloids have an 8,9 or 9,10 double bond within the fourth ring of the tetracyclic ergoline structure. Ergot alkaloid production also is associated with the distantly related opportunistic human pathogen, Aspergillus fumigatus, in the order Eurotiales $(4,7)$. Aspergillus fumigatus ergot alkaloid biosynthesis renders simple clavines and differs significantly from the more complex profile of C. purpurea and Neotyphodium spp., with one important distinction being the saturation of the fourth ring of the ergoline structure (Fig. 1).

Several genes involved in the ergot alkaloid pathways of A. fumigatus and clavicipitaceous fungi are found clustered together within the genome of each species (2, $3,5,19)$. These distantly related fungi are hypothesized to share several early pathway steps, after which the pathways diverge to yield distinct sets of ergot alkaloids $(12,15)$. 
The gene, dmaW, which encodes for a dimethylallyltryptophan (DMAT) synthase in Neotyphodium, functions similarly in A. fumigatus $(2,18,20,21)$. Annotation of the regions surrounding this gene in A. fumigatus has identified potential homologues of genes also found in N. coenophialum and C. purpurea ergot alkaloid gene clusters (2). One of the shared genes, eas $A$, is predicted to encode an old yellow enzyme (OYE). Old yellow enzymes are flavin-containing oxidoreductases initially found in brewers' bottom yeast (22). Enzymes in this family use a reduced flavin cofactor and an active site tyrosine residue to reduce an $\alpha / \beta$-unsaturated aldehyde or ketone. Subsequently, the enzymes use NADPH to restore the flavin cofactor to its reduced state. OYEs catalyze multiple reactions useful for both biotechnological and pharmaceutical applications; however physiological roles and natural substrates for many of these enzymes presently are unknown. Based on the apparent need in the ergot alkaloid pathway of A. fumigatus for reduction of a carbon-carbon double bond in the intermediate chanoclavine aldehyde, we hypothesized that the OYE-encoding gene eas $A$ is required for ergot alkaloid biosynthesis $(2,15)$. In this study, knockout and complementation analyses of eas $A$ in $A$. fumigatus are performed to ascertain the role of this gene product in ergot alkaloid biosynthesis.

\section{MATERIALS AND METHODS}

Fungi and culture conditions. Aspergillus fumigatus isolate FGSC A1141 (13) and its mutant derivatives were cultured on potato dextrose agar (PDA; $20 \mathrm{~g}$ dehydrated potato,

$20 \mathrm{~g}$ glucose, and $15 \mathrm{~g}$ agar per liter). Liquid cultures used for preparation of protoplasts or DNA were grown at $37{ }^{\circ} \mathrm{C}$ on an orbital shaker $(150 \mathrm{rpm})$ overnight in a $250 \mathrm{~mL}$ 
Erlenmeyer flask containing $100 \mathrm{~mL}$ of malt extract medium $(50 \mathrm{~g}$ malt extract, $50 \mathrm{~g}$ lactose, $5 \mathrm{~g}$ asparagine, and $0.4 \mathrm{~g} \mathrm{~K}_{2} \mathrm{HPO}_{4}$ per L).

Disruption and complementation of easA. A 595-bp fragment within the coding sequence of eas $A$ was amplified by PCR, primed with oligonucleotides oyeF (5'ACCGAAGCAACAGACATCACC-3') and oyeR (5'-ATATGCGACATCCAATCGCC3'). The $50 \mu \mathrm{L}$ PCR reaction contained $50 \mathrm{mM} \mathrm{KCl}, 10 \mathrm{mM}$ Tris- $\mathrm{HCl}(\mathrm{pH} 9.0), 0.1 \%$ (v/v) Triton X-100, $1.5 \mathrm{mM} \mathrm{MgCl}_{2}, 200 \mu \mathrm{M}$ of each deoxyribonucleotide triphosphate, 1 $\mu \mathrm{M}$ of each primer, and 2.5 units of Taq DNA polymerase (Promega, Madison, Wisconsin), which was added once the thermocycler reached $95^{\circ} \mathrm{C}$ in the initial denaturing period. The reaction began with an initial denaturing step of $2 \mathrm{~min} 30 \mathrm{sec}$ at $95^{\circ} \mathrm{C}$, followed by 35 cycles of $1 \mathrm{~min}$ at $94{ }^{\circ} \mathrm{C}, 1 \mathrm{~min}$ at $55^{\circ} \mathrm{C}$, and $1 \mathrm{~min}$ at $72{ }^{\circ} \mathrm{C}$, with a final extension step of $72^{\circ} \mathrm{C}$ for $7 \mathrm{~min}$. A 4.5-kb disruption construct, pAFOYE1, was generated by ligating the PCR product into the T/A overlap vector pCR2.1 (Invitrogen, Carlsbad, California). Prior to transformation into the fungus, pAFOYE1 was linearized by a restriction digest at the unique $S m a$ I site within the eas $A$ region of the construct. Protoplast preparation and transformation were preformed as described by Murray et al. (10), with modifications previously noted by Coyle and Panaccione (2). Protoplasts were separated from undigested hyphae by means of filtration through cheesecloth instead of step-gradient centrifugation. Protoplasts in the filtrate were then pelleted by centrifugation (5 min, $3436 \mathrm{xg}$ ) and washed twice with $0.7 \mathrm{M} \mathrm{NaCl}$ and once in STC (10) prior to a final resuspension in STC. Linearized disruption construct pAFOYE1 (Fig. 2A) and a hygromycin resistance plasmid, pMOcosX (11), linearized at a unique NotI site (2) were co-transformed into the protoplasts. Transformants colonies were purified to a 
homokaryotic state by culturing from single spores. These cultures then were screened in three different PCR assays to identify strains in which the disruption construct inserted within the native easA. With the exception of different primers and annealing temperatures, the PCR reactions are as described above. The 5' border of the homologous recombination event was confirmed by PCR (annealing temperature at $57^{\circ} \mathrm{C}$ ) primed with oligonucleotides UR (5'- AGCTATGACCATGATTACGCCA -3', which anneals to vector sequences near the universal primer annealing site in pCR2.1) and oyeFscrn (5'-GGAAGATGTCATCTCCAACATAG-3', which is complementary to eas $A$ sequences near the 5 '-end of the eas $A$ gene and flanking the intended site of integration) (Fig. 2A). The juxtaposition of sequences near the $3^{\prime}$ border of the integration event was verified by PCR (annealing temp $57^{\circ} \mathrm{C}$ ) primed from oligonucleotides UF (5'- GCCAGTGAATTGTAATACGACTC -3', complementary to vector sequences near the reverse primer annealing site of pCR2.1), and oyeRscrn (5'AGCCTTCTCTTGATAGCGTGCT-3', which anneals to easA sequences near the 3'-end of the gene and flanking the intended site of integration) (Fig. 2A). PCR across the entire eas $A$ locus was conducted by priming with oligonucleotides oyeFscrn and oyeRscrn (both described above) at an annealing temperature of $57^{\circ} \mathrm{C}$.

Integration of pAFOYE1 into the native eas $A$ also was analyzed by Southern hybridization as described previously (2). Exceptions were inclusion of an easA-specific probe generated by amplifying wild-type $A$. fumigatus genomic DNA in a reaction primed with oyeF and oyeR according to the PCR conditions described above, and substitution of $1 \times$ DIG DNA Labeling Mix (Roche, Mannheim, Germany) for unlabeled dNTPs. Genomic DNA was digested by SalI prior to Southern blot analysis. 
The eas $A$ knockout (ko) strain was complemented by ectopic insertion of a functional copy of $A$. fumigatus eas $A$ into the mutant genome (eas $A \mathrm{ct}$ ). This $3.3-\mathrm{kb}$ fragment contained the entire coding sequence along with approximately $1.2-\mathrm{kb}$ of $5^{\prime}-$ flanking sequences and 1.0-kb of 3'-flanking sequences and was amplified by PCR primed from WeasAF (5'-AGAATCGAGGTGAGCGTGTAGAATGC-3') and WeasAR (5'-AAGCTGGAACACTACTGATCGAGTGC-3'). Reaction conditions were similar to those previously described by Coyle et al. (1) except for an annealing temperature of $58^{\circ} \mathrm{C}$ and an extension period of $3 \mathrm{~min} 30 \mathrm{sec}$.

Additionally, the eas $A$ ko strain was complemented with the eas $A$ homologue from Claviceps purpurea strain ATCC20102. Amplification of this gene was primed from CpoyeF (5'-TGAGTTCGAACGATGATCTGTAGCG -3') and CpoyeR (5'TGAGTTCGAAAGAGTCTTCTACGCC -3'), with PCR conditions as described above, except for an annealing temperature of $54{ }^{\circ} \mathrm{C}$ and an extension time of $2 \mathrm{~min} 30 \mathrm{sec}$. The PCR product was co-transformed into an easA-disrupted mutant of $A$. fumigatus along with a phleomycin resistance plasmid, pBC-phleo (Fungal Genetics Stock Center, University of Missouri-Kansas City, Kansas City, MO) linearized at a unique NotI site. Transformants were selected on complete regeneration medium (14) containing 100 $\mu \mathrm{g} / \mathrm{mL}$ phleomycin and incubated at $37^{\circ} \mathrm{C}$. Complemented transformants were cultured from individual germinated conidia in order to achieve a homonucleate culture. To test for the presence of the original disruption construct, PCR reactions were conducted as described above, primed with oyeFscrn and UR (for the 5' flank of the integration) and UF and oyeRscrn (for the 3' flank). An additional PCR screen was performed with either primers WeasAF and WeasAR (described above) or CPoyeF and CpoyeR (described 
above), to test for the presence of the introduced wild-type or C. purpurea allele in the complemented transformants ( $\mathrm{Cp}$ eas $A \mathrm{ct})$.

Sequencing of easA from Claviceps spp. Amplification of the C. purpurea strain ATCC20102 homologue of easA is described above, except using A. fumigatus eas $A$ complemented strain as template DNA. Internal portions (corresponding to coding sequences for amino acids 19-341 based on the C. purpurea P1 (accession number CAG28312 sequence) of the Claviceps gigantea and Claviceps africana eas A homologues were amplified in reactions primed with degenerative oligonucleotides ClavoyeF (5'- CACAAARTYGTRCTYTCTCCYATG -3') and ClavoyeR (5'YGTGTCTCGRTTGTACTTYTGCAG -3'). The reactions were described above, except for an annealing temperature of $48^{\circ} \mathrm{C}$ and extension period of $2 \mathrm{~min}$. PCR products were ligated into the pCR2.1 vector. Sequencing was performed by Davis Sequencing (www.davissequencing.com, Davis, CA). The initial round of sequencing for all three Claviceps spp. was primed from universal primers M13F and M13R. To complete double-stranded sequence from the longer C. purpurea eas $A$ clone, a second round of sequencing was primed with custom primers CpoyeM13F2(5'GATCAAGCAGCGAAGTACCG -3') and CpoyeM13R2 (5'TTGGAGGACTGGTCATGAGG -3’).

Sequences were aligned with additional available EasA sequences from ergot alkaloid producing fungi (C. purpurea strain P1 (accession number CAG28312), Neotyphodium lolii (accession number ABM91449), Claviceps fusiformis (accession number ABV57819), and Epichloë festucae, contig 3372 nucleotides 256-1251 (reverse, complement) within the available E. festucae genome 
(http://www.genome.ou.edu/blast/ef blastall.html), determined by tblastn with N. lolii EasA sequence corresponding to amino acids 19-350, by application ClustalW at the European Biotechnology Institute (EBI) web site (www.ebi.ac.uk/ClustalW/). The alignment was analyzed in a distance analysis at the same EBI site (accepting defaults of all parameters) to construct a Neighbor-Joining tree.

Analysis of ergot alkaloids. To analyze ergot alkaloid production, small cubes (containing approximately $8 \mathrm{~mm} \times 8 \mathrm{~mm}$ of colony surface area) were removed from sporulating colonies that had been grown on PDA at $37^{\circ} \mathrm{C}$ for 5 days. Samples were submerged in $0.4 \mathrm{~mL}$ of $80 \%$ methanol, vortexed briefly, rotated end-over-end (44 rpm) for $2 \mathrm{hr}$, and centrifuged (10 min., 14,000 x g) to pellet fungal mass and agar. Supernatants were analyzed by HPLC (C18 column) with acetonitrile-ammonium acetate gradients and detected with dual fluorescence detectors set at excitation and emission wavelengths of $272 \mathrm{~nm} / 372 \mathrm{~nm}$ and $310 \mathrm{~nm} / 410 \mathrm{~nm}(1,13)$. The identities of peaks corresponding to chanoclavine, festuclavine, and fumigaclavines $\mathrm{A}, \mathrm{B}$, and $\mathrm{C}$ were previously established by mass spectral analyses of native and de-esterified fractions (13). These peak identities also were confirmed by their elimination in extracts from $d m a W$, a strain in which the determinant step of the ergot alkaloid pathway was inactivated (2). Chanoclavine, agroclavine, setoclavine, and isosetoclavine were identified by coelution with standards, and their masses were confirmed by LC-MS (see below).

To quantify ergot alkaloids and conidiation from the wild type, eas $A$ ko and $\mathrm{Cp}$ eas $A$ ct samples, cultures were grown on PDA for 5 days at $37^{\circ} \mathrm{C}$. Samples, 
containing $50.3 \mathrm{~mm}^{2}$ of colony surface area, were excised from cultures with the broader end of a disposable 1-mL pipette tip (Fisher Scientific, catalog number 02-681-172), submerged in $1 \mathrm{~mL} 80 \%$ methanol, agitated by vortexing, and rotated at $44 \mathrm{rpm}$ for $2 \mathrm{hr}$. Aliquots were removed for HPLC analyses and for quantification of conidia. Concentrations of agroclavine, setoclavine, and isosetoclavine were based on peak area relative to the external standards of agroclavine (Sigma, St. Louis, MO) (for agroclavine) and ergotamine (for setoclavine and isosetoclavine) as previously described (Panaccione and Coyle 2005). To determine spore counts, dilutions of the methanol extracts were prepared and conidia were enumerated with a hemacytometer and light microscope. Five samples were analyzed for each treatment. Data from eas $A$ ko and C. purpurea easA complemented strains were compared by Wilcoxon/Krustal-Wallis tests (rank sums). Extracts for LC-MS analyses were prepared from cultures inoculated on the surface of liquid potato dextrose medium (PDA without agar, $50 \mathrm{~mL}$ in a $250-\mathrm{mL}$ Erlenmeyer flask) and grown at $37^{\circ} \mathrm{C}$ with no agitation. Ergot alkaloids were extracted by agitation in $4 \mathrm{~mL}$ of $100 \%$ methanol. The methanol and dislodged conidia and hyphal fragments were transferred to microcentrifuge tubes, vortexed, and rotated end-over-end for a 2 -hr period. Then the sample was centrifuged (10 min., 14,000 x g) to pellet fungal material. Methanol extracts and individual peaks collected from HPLC analysis performed as described above were concentrated under vacuum and sent to Massachusetts Institute of Technology for liquid chromatography-mass spectrometry (LC-MS) analyses. LC-MS samples were separated on an Acquity Ultra Performance BEH C18 column with a 1.7 micrometer particle size, and 2.1 x $100 \mathrm{~mm}$ dimensions and were eluted with a linear gradient $(0.5 \mathrm{~mL} / \mathrm{min})$ of 20 parts acetonitrile +80 parts $0.1 \%$ 
formic acid in water, initially, to 80 parts acetonitrile +20 parts $0.1 \%$ formic acid in water at 5 min. Masses of eluates were detected by a Micromass LCT Premier TOF MS with an electrospray ionization source.

\section{RESULTS}

Disruption and complementation of easA. Integration of the disruption construct pAFOYE1 into the native eas $A$ gene of $A$. fumigatus via homologous recombination occurred in two out of the 64 hygromycin-resistant transformants. Insertion of pAFOYE1 within easA was verified by PCR, with screens of the 5' and 3 ' borders of the integration resulting in 811-bp and 992-bp fragments, respectively (Fig 2B). In the mutant, no 1-kb amplification product was detected when PCR was primed with oligonucleotides with binding sites separated from each other by $1-\mathrm{kb}$ in the wild type due to the insertion of the disruption construct within the eas $A$ locus (Fig 2B). Southern blot analysis showed multiple insertions of pAFOYE1 within eas $A$ by the shift in mobility of the eas $A$-specific region (Fig 2C).

The eas $A$ mutants did not produce festuclavine or fumigaclavines $\mathrm{A}, \mathrm{B}$, and $\mathrm{C}$, which are four ergot alkaloids typically detectable in A. fumigatus (13). However, an important intermediate within the ergot alkaloid pathway, chanoclavine, which eluted from the HPLC column at 32.8 min, was observed in the mutant's chemical profile (Fig 3 \& 4). In addition, a $255.05 \mathrm{~m} / \mathrm{z}$ fragment was observed by mass spectrometry within the eas $A$ ko extract, although elution time by HPLC was unknown (not shown). Trace quantities of agroclavine, setoclavine, and isosetoclavine were identified in mutant extracts by coelution with standards (Fig. 3). Co-transformation of a copy of $A$. fumigatus easA restored normal ergot alkaloid production. This wild-type allele inserted 
ectopically within the genome. The construct, pAFOYE1, was verified to be inserted within the native easA locus by PCR screens of the recipient strain (Fig. 2B).

\section{Complementation of eas A mutant with easA homologue from C. purpurea.}

Ectopic insertion of the eas $A$ homologue from $C$. purpurea resulted in accumulation of larger quantities of agroclavine, setoclavine and its isomer isosetoclavine within the complemented strain (Table 1, Fig. 3). The complemented strain was positive for the presence of the knockout construct, pAFOYE1 (not shown).

Analyses of eas $A$ sequences among ergot alkaloid producers. The translated sequence of $A$. fumigatus eas $A$ was $57 \%$ similar to EasA homologues of $N$. lolii, $C$.

fusiformis, and C. purpurea. A. fumigatus eas $A$ consists of $1.1 \mathrm{~kb}$ of coding sequence uninterrupted by introns and has a tyrosine residue at the active site of the enzyme, as defined by analyses of Kluyveromyces marxianus old yellow enzyme. The eas $A$ homologues of $C$. gigantea and $C$. africana (fungi that, like A. fumigatus, produce festuclavine and derivatives of festuclavine with a saturated "D" ring) also contain tyrosine at the active site (Fig. 5). The eas $A$ homologues of $N$. lolii, E. festucae, $C$. fusiformis, and two isolates of C. purpurea (all of which produce clavines with unsaturated D rings) all have a phenylalanine substituted for the active site tyrosine (Fig 5). Based on overall amino acid sequence identity, the easA gene of $A$. fumigatus is most distant from the eas $A$ genes of the other festuclavine-producing fungi ( $C$. africana and $C$. gigantea) (Fig. 6).

\section{DISCUSSION}

Disruption and complementation analyses of eas $A$ conducted in this study demonstrate that this gene is required for ergot alkaloid biosynthesis in A. fumigatus. Disruption of 
eas $A$ in A. fumigatus halted the conversion of chanoclavine into festuclavine. Besides chanoclavine, this mutant also accumulated an intermediate with a molecular weight of 254.05. This intermediate is hypothesized to be chanoclavine aldehyde, an oxidized form of chanoclavine likely to serve as the substrate for the eas $A$ product. However, the product was not present in sufficient concentration to perform further structural analyses. Chanoclavine aldehyde was hypothesized to be a transitional step between chanoclavine and festuclavine in the A. fumigatus ergot alkaloid biosynthetic pathway, which also was suggested by our knockout analysis and appears to be the last common intermediate between the A. fumigatus and ergot pathways prior to their divergence (Fig 7).

The chemical profile of the eas $A$ knockout showed the presence of small quantities of agroclavine and its isomeric oxidation products setoclavine and isosetoclavine. In total, these alkaloids were present at a concentration less than 0.03 fg/conidium (Table 1). We hypothesize that chanoclavine aldehyde, which accumulates due to the knock out of easA, tautomerizes to its enol form, thus allowing rotation of the aldehyde group attached at carbon 8 into close proximity to the $\mathrm{N}$ at position 6 for ring closure via a Schiff's base reaction. Since the 8,9 double bond was not reduced (due to the lack of Eas A activity), agroclavine as opposed to festuclavine is the product. Agroclavine produced in this way may then be acted on by endogenous peroxidases to yield setoclavine and isosetoclavine. The oxidation of agroclavine to yield setoclavine and its isomers by peroxidases from a variety of sources has been demonstrated numerous times (16). Pseudomonas aeruginosa and peroxidases from homogenates of plants, such as tomatoes, potato sprouts, and morning glory seedlings, have been shown to conduct this bioconversion (8). Complementation of the A. fumigatus easA knockout 
with C. purpurea easA resulted in a significant increase in agroclavine, setoclavine, and isosetoclavine (Table 1).

While the A. fumigatus easA homologue may closely resemble the gene sequence of Claviceps purpurea easA, they differ in their enzymatic capabilities. Both eas $A$ homologues resembles OYE, the flavin-dependent oxidoreductase (2). Data from this study suggest that the pathways diverge after chanoclavine aldehyde, due to the differing enzymatic properties of EasA. Chanoclavine aldehyde is an example of an $\alpha, \beta-$ unsaturated aldehyde, a suitable substrate for OYE, which would have the capability to reduce the 8,9 double bond in chanoclavine aldehyde, allowing rotation of the aldehyde group to form dihydrochanoclavine aldehyde, and positions the aldehyde group close to the N-methyl group (22). Formation of the $\mathrm{C}-\mathrm{N}$ bond and closure of the fourth ring causes loss of $\mathrm{H}_{2} 0$, converting dihydrochanoclavine aldehyde into festuclavine, which is observed in A. fumigatus (15) (Fig 7). Through complementation studies, our data suggest that C. purpurea EasA only temporarily reduces the 8,9 double bond in chanoclavine aldehyde so that agroclavine is synthesized instead of festuclavine. Agroclavine can be synthesized into setoclavine and its isomer by numerous peroxidases (16). Chemical analyses indicate that peroxidases endogenous to A. fumigatus are capable of this conversion due to the accumulation of these two products (Fig. $3 \& 4$ ) OYEs have been studied intensively in yeasts, and much is known about their mechanism and important active site residues $(6,9,22)$. OYEs reduce their substrates by a two-step mechanism. Initially a hydride ion is transferred from the reduced flavin cofactor to the $\beta$ carbon (relative to the aldehyde or ketone). Subsequently, a proton is donated by $\mathrm{Y}_{196}$ which sits at the active site of the enzyme (9). Yeast mutants with F 
substituted for $\mathrm{Y}$ at that position transfer the hydride ion but never the second proton (6, 9, 22). Interestingly, C. purpurea EasA does not contain tyrosine at this position within its sequence and the phenylalanine substitution may be responsible for the differing enzymatic activity between the homologues. The phenylalanine is incapable of donating the necessary hydrogen to the substrate. Therefore, the enzyme cannot complete the reduction reaction even though the hydride ion was already transferred to the substrate, allowing temporary rotation of the 8,9 double bond in chanoclavine aldehyde. Rotation around this bond can occur spontaneously, but most likely occurs at a slow rate. This can be observed in the dramatic difference in accumulation between eas $A$ ko and $\mathrm{Cp}$ eas $A$ ct strains. The much smaller quantities of agroclavine and setoclavine and isosetoclavine observed in the easA ko prior to complementation are hypothesized to arise after noncatalyzed isomerization in the enol tautomer of chanoclavine aldehyde, more of which must accumulate in the eas $A$ ko (Fig. $3 \& 8$ ).

Interestingly, N. lolii, E. festucae, and C. fusiformis, all agroclavine producers, have phenylalanine at position 196, whereas those which synthesize festuclavine, such as C. gigantea and C. africana, have a tyrosine at this specific residue. Notably, $A$. fumigatus eas $A$ is most distant from the eas $A$ homologues of other festuclavine producers, C. africana and C. gigantea. Therefore, even though the presence of the $\mathrm{Y}_{196}$ is common among all festuclavine producers, there are other differences among the eas $A$ sequences affecting their phylogenetic relationships (Fig. 5 \& 6).

The gene eas $A$ is believed to be the branch point between the two lineage-specific pathways, making chanoclavine aldehyde the last common intermediate. Whether the 
presence of tyrosine or phenylalanine at the amino acid residue 196 is the only basis for production of festuclavine versus agroclavine still needs to be investigated further.

\section{ACKNOWLEDGEMENTS}

We thank Johnathan Cheng and Dr. Sarah O’Connor (Massachusetts Institute of Technology) for performing mass spectrum analyses and for helpful suggestions on pathway intermediates. We thank Dr. Paul Tooley, USDA ARS, Frederick, MD, for generously providing DNA from $C$. africana and $C$. gigantea. 


\section{REFERENCES}

1. Coyle, C. M., S. C. Kenaley, W. R. Rittenour, and D. G. Panaccione. 2007. Association of ergot alkaloids with conidiation in Aspergillus fumigatus. Mycologia 99:804-811.

2. Coyle, C. M., and D. G. Panaccione. 2005. An ergot alkaloid biosynthesis gene and clustered hypothetical genes from Aspergillus fumigatus. Appl. Environ. Microbiol. 71:3112-3118.

3. Fleetwood, D. J., B. Scott, G. A. Lane, A. Tanaka, and R. D. Johnson. 2007. A complex ergovaline gene cluster in Epichloe endophytes of grasses. Appl. Environ. Microbiol. 73:2571-2579.

4. Flieger, M., M. Wurst, and R. Shelby. 1997. Ergot alkaloids -- sources, structures and analytical methods. Folia Microbiol. 42:3-30.

5. Haarmann, T., C. Machado, Y. Lübbe, T. Correia, C. L. Schardl, D. G. Panaccione, and P. Tudzynski. 2005. The ergot alkaloid gene cluster in Claviceps purpurea: extension of the cluster sequence and intra species evolution. Phytochemistry 66:1312-1320.

6. Kohli, R. M., and V. Massey. 1998. The oxidative half-reaction of old yellow enzyme. The role of tyrosine 196. J. Biol. Chem. 273:32763-32770.

7. Kozlovsky, A. G. 1999. Producers of ergot alkaloids out of Claviceps genus, p. 479-499. In V. Kren and L. Cvak (ed.), In Ergot: The Genus Claviceps. Harwood Academic Publishers, The Netherlands. 
8. Křen, V. 1999. Biotransformation of ergot alkaloids, p. 229-266. In V. Kren and L. Cvak (ed.), In Ergot: The Genus Claviceps. Harwood Academic Publishers, The Netherlands.

9. Meah, Y., and V. Massey. 2000. Old Yellow Enzyme: Stepwise reduction of nitro-olefins and catalysis of aci-nitro tautomerization. Proc. Natl. Acad. Sci. USA 97:10733-10738.

10. Murray, F. R., G. C. M. Latch, and D. B. Scott. 1992. Surrogate transformation of perennial ryegrass, Lolium perenne, using genetically modified Acremonium endophyte. Mol. Gen. Genet. 233:1-9.

11. Orbach, M. J. 1994. A cosmid with a HyR marker for fungal library construction and screening. Gene 150:159-162.

12. Panaccione, D. G. 2005. Origins and significance of ergot alkaloid diversity in fungi. FEMS Microbiol. Lett. 251:9-17.

13. Panaccione, D. G., and C. M. Coyle. 2005. Abundant respirable ergot alkaloids from the common airborne fungus Aspergillus fumigatus. Appl. Environ. Microbiol. 71:3106-3111.

14. Panaccione, D. G., R. D. Johnson, J. Wang, C. A. Young, P. Damrongkool, B. Scott, and C. L. Schardl. 2001. Elimination of ergovaline from a grassNeotyphodium endophyte symbiosis by genetic modification of the endophyte. Proc. Natl. Acad. Sci. USA 98:12820-12825.

15. Panaccione, D. G., C. Schardl, and C. M. Coyle. 2006. Pathways to diverse ergot alkaloid profiles in fungi, p. 23-52 In J. T. Romeo (ed.), Recent Advances in Phytochemistry, vol. 40. Elsevier, Amsterdam, The Netherlands. 
16. Panaccione, D. G., B. A. Tapper, G. A. Lane, E. Davies, and K. Fraser. 2003. Biochemical outcome of blocking the ergot alkaloid pathway of a grass endophyte. J. Agric. Food Chem. 51:6429-6437.

17. Schardl, C., D. G. Panaccione, and P. Tudzynski. 2006. Ergot alkaloids-biology and molecular biology, p. 45-86. In G. A. Cordell (ed.), Alkaloids Chem. Biol., vol. 63. Academic Press, San Diego, CA.

18. Tsai, H.-F., H. Wang, J. C. Gebler, C. D. Poulter, and C. L. Schardl. 1995. The Claviceps purpurea gene encoding dimethylallyltryptophan synthase, the committed step for ergot alkaloid biosynthesis. Biochem. Bioph. Res. Co. 216:119-125.

19. Tudzynski, P., K. Holter, T. Correia, C. Arntz, N. Grammel, and U. Keller. 1999. Evidence for an ergot alkaloid gene cluster in Claviceps purpurea. Mol. Gen. Genet. 261:133-141.

20. Unsöld, I. A., and S.-M. Li. 2005. Overproduction, purification and characterization of FgaPT2, a dimethylallyltryptophan synthase from Aspergillus fumigatus. Microbiology 151:1499-1505.

21. Wang, J., C. Machado, D. G. Panaccione, H.-F. Tsai, and C. L. Schardl. 2004. The determinant step in ergot alkaloid biosynthesis by an endophyte of perennial ryegrass. Fungal Genet. Biol. 41:189-198.

22. Williams, R. E., and N. C. Bruce. 2002. 'New uses for an old enzyme' - the old yellow enzyme family of flavoenzymes. Microbiology 148:1607-1614. 


\section{FIGURE LEGENDS}

Figure 1: Examples of ergot alkaloids. Festuclavine is in the clavine class of alkaloids and is synthesized by A. fumigatus, whereas ergotamine is an ergopeptine, and is produced by Claviceps spp. The letters, A-D, labeled within festuclavine, identify the four rings common to ergot alkaloids. The atoms within this four ring structure are numbered 1-15.

Figure 2: easA ko disruption strategy. (A) Knockout construct, pAFOYE1, contains an internal fragment of eas $A$ and was linearized at $S m a \mathrm{I}$ site prior to transformation. This diagram shows tandem integration of three constructs within ko. UF $=$ annealing site for primer derived from universal primer sequences in vector; $3 \mathrm{~S}=$ annealing site for primer flanking the $3^{\prime}$ border of integration site; $\mathrm{UR}=$ annealing site for primer derived from reverse primer sequences in vector; $5 \mathrm{~S}=$ annealing site for primer flanking the $5^{\prime}$ border of integration site. (B) Primers $5 \mathrm{~S}$ and $3 \mathrm{~S}$ primed amplification of a 1.0-kb fragment only in the wild-type isolate. Primers UR and 5S primed amplification of the expected $0.8-\mathrm{kb}$ band from strains with disrupted eas $A$ allele but not from the wild type. Primers UF and $3 \mathrm{~S}$ primed amplification of the indicated $1.0-\mathrm{kb}$ product from eas $A$ ko and eas $A$ complemented (easA ct). Relative mobility of relevant fragments (sizes in kb) of BstEIIdigested bacteriophage lambda is indicated. (C) Integration of transforming DNA at only the targeted site was confirmed by Southern blot hybridization. DNA extracts were digested with SalI and hybridized with a digoxigenin-dUTP labeled eas $A$ probe. 
Figure 3: Chromatogram of wild-type A. fumigatus (wt), eas $A$ ko, and eas $A$ ko complemented with a functional copy of $C$. purpurea eas $A(\mathrm{Cp}$ eas $A \mathrm{ct}) . \mathrm{B}=$ fumigaclavine $\mathrm{B} ; \mathrm{F}=$ festuclavine; $\mathrm{A}=$ fumigaclavine $\mathrm{A} ; \mathrm{C}=$ fumigaclavine $\mathrm{C} ; \mathrm{I}=$ isosetoclavine; $\mathrm{S}=$ setoclavine; $\mathrm{AG}=$ agroclavine; $\mathrm{CH}=$ chanoclavine. Excitation and emission wavelengths are provided in the upper left corner of each chromatogram.

\section{Figure 4: ESI-MS of $A$. fumigatus eas $A$ ko strain and its $C$. purpurea eas $A$}

complemented strain. (A) Mass spectrum of the major 32-min peak that accumulates in eas $A$ ko and coelutes with chanoclavine during HPLC analysis. (B) Mass spectra of three fractions collected from HPLC analysis of extract from Cp eas $A$ ct. The 44-min peak coeluted with agroclavine; the 39-min peak and 42-min peak coeluted with setoclavine and isosetoclavine, respectively.

Figure 5: Alignment of EasA sequences. Partial protein sequences, EasA, for $A$. fumigatus, C. africana, C.fusiformis, C. gigantea, C. purpurea (P1 and 20102), E. festucae, and N. lolii, were aligned by ClustalW. The residue of interest (highlighted in yellow) corresponding to the active site tyrosine (Y) is as expected for A. fumigatus, $C$. africana, and $C$. gigantea, whereas all remaining sequences have a phenylalanine $(\mathrm{F})$ at this position.

Figure 6: Distance analysis of EasA homologues among ergot alkaloid producers. (A) Distance matrix based on alignment of EasA sequences. The furthest distance is 
between A. fumigatus and C. gigantea. (B) Unrooted Neighbor-Joining tree derived from the distance matrix calculated from aligned EasA sequences.

Figure 7: The branch point of the ergot alkaloid pathway. The pathways diverge after chanoclavine aldehyde, due to the differing enzymatic properties of easA. The eas $A$ product in A. fumigatus reduces the vinyl group in chanoclavine aldehyde, allowing rotation of the aldehyde group into close proximity of the $\mathrm{N}$-methyl group for ring closure. The product of $C$. purpurea eas $A$ only temporarily reduces the double bond resulting in formation of agroclavine instead of festuclavine. Setoclavine and its isomer are readily produced from agroclavine by peroxidases from multiple sources, in this case presumably endogenous $A$. fumigatus peroxidases (16). DMAPP $=$ dimethylallylpyrophosphate; $\mathrm{PPO}=$ pyrophosphate $; \operatorname{Trp}=$ tryptophan; AdoMet $=\mathrm{S}-$ adenosylmethionine; DMAT $=$ dimethylallyltryptophan .

\section{Figure 8: Model for noncatalyzed closure of $D$ ring via keto-enol tautomerism.}

Chanoclavine aldehyde undergoes movement of electrons within its double bonds, creating the intermediary enolate ion, which acquires hydrogen from solvent to form enol tautomer. This change in double bond location allows rotation around the alpha carbon, positioning the hydroxyl group within close proximity to the amine group. Loss of the proton and reformation of the aldehyde creates isochanoclavine aldehyde. The carbonyl group interacts with the amine group, and water is lost, resulting in closure of D ring as an iminium ion. An additional NADPH-dependent reductase is required to reduce the iminium ion to agroclavine (17). 
Table 1. Accumulation of agroclavine, setoclavine, and isosetoclavine in A. fumigatus eas $A$ ko and $A$. fumigatus eas $A$ ko complemented with C. purpurea easA

\begin{tabular}{|c|c|c|}
\hline \multirow[b]{2}{*}{ Source } & \multicolumn{2}{|c|}{ Fg ergot alkaloid per conidium (mean \pm standard error) } \\
\hline & Agroclavine $^{1}$ & Setoclavine / Isosetoclavine \\
\hline wild type & $\mathrm{nd}^{2}$ & nd \\
\hline eas $A$ ko & $0.0095 \pm 0.0027 \mathrm{a}$ & $0.012 \pm 0.0019 \mathrm{a}$ \\
\hline $\mathrm{Cp}$ eas $A$ ct & $1.1 \pm 0.082 \mathrm{~b}$ & $1.4 \pm 0.090 \mathrm{~b}$ \\
\hline \multicolumn{3}{|c|}{ by Wilcoxon/Kruskal-Wallis Tests; means with different letter indicate } \\
\hline${ }^{2}$ nd, none de & ted & \\
\hline
\end{tabular}

Chapter 3 - Table 1 


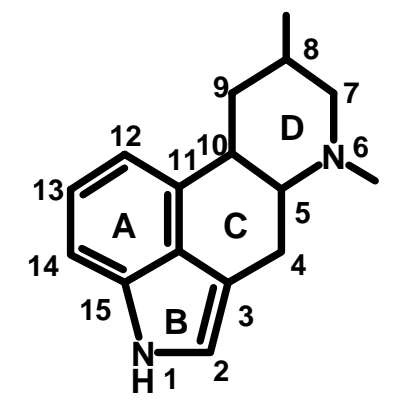

\section{Festuclavine}

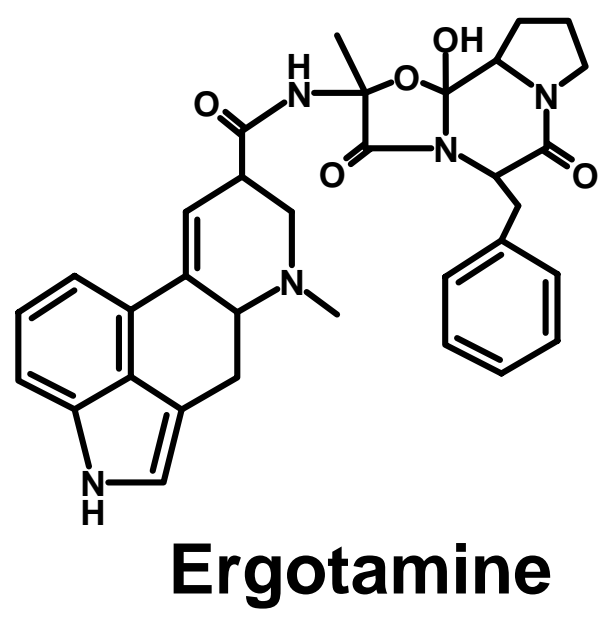

Chapter 3 - Figure 1 


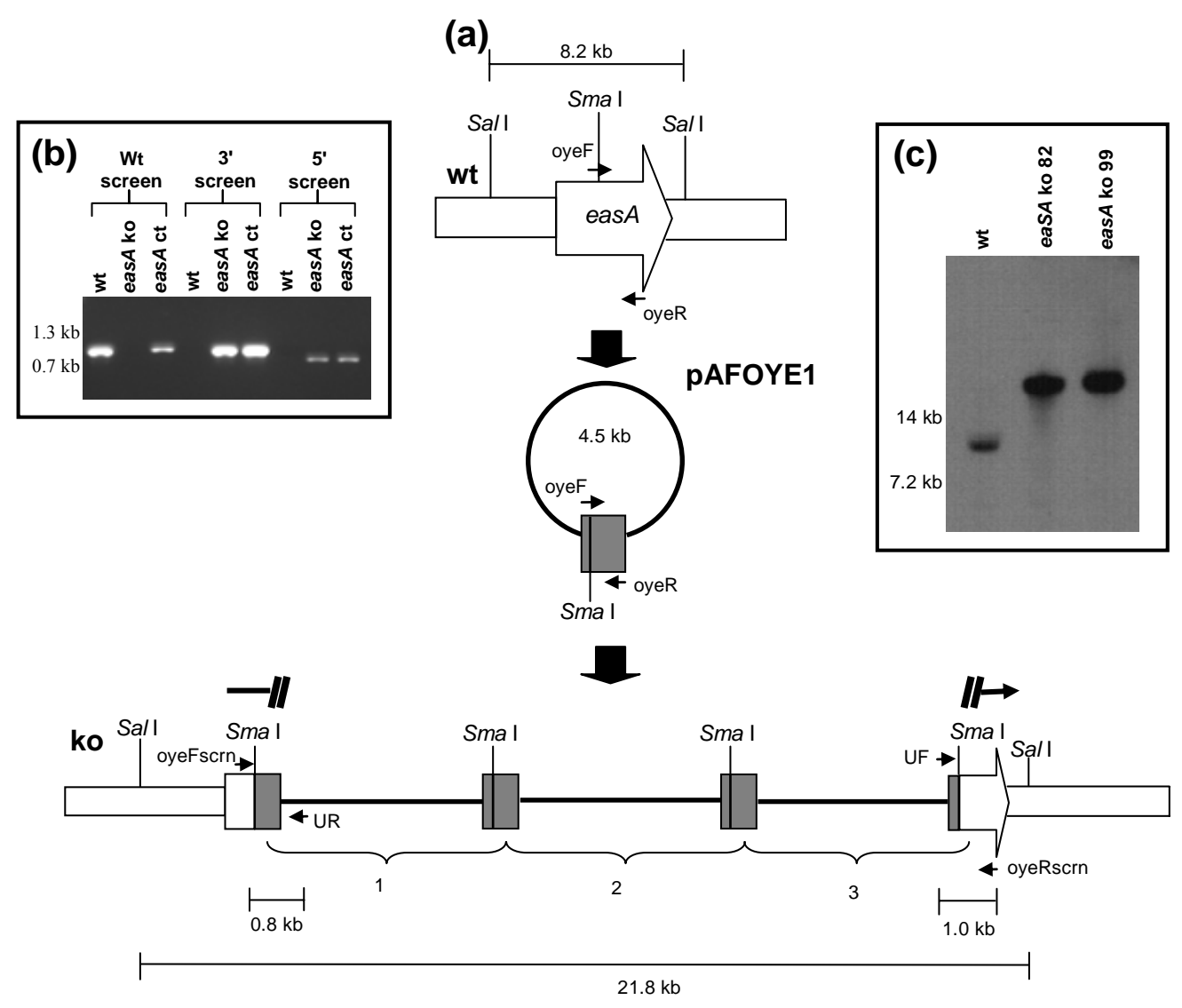

Chapter 3 - Figure 2 

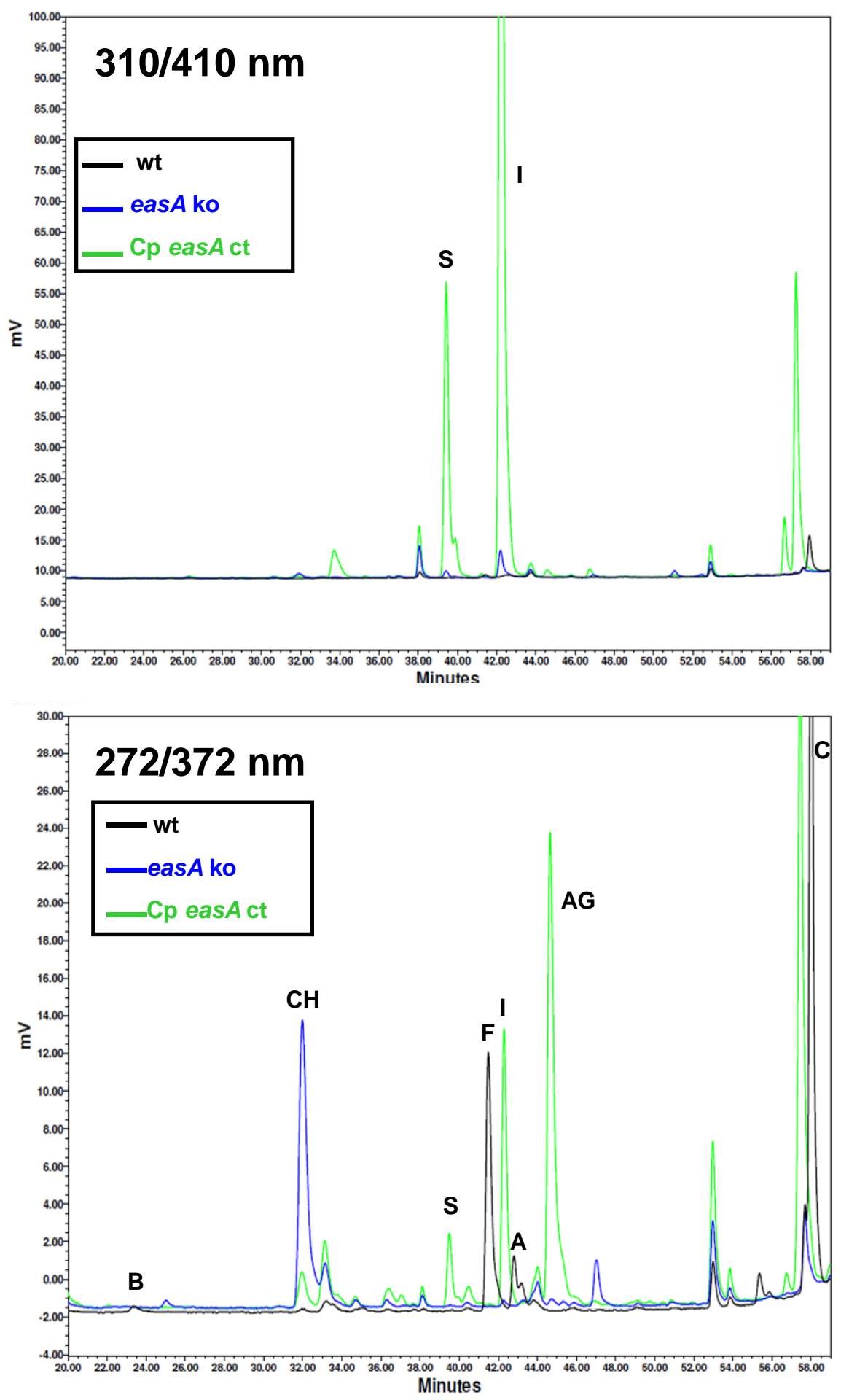

\section{Chapter 3 - Figure 3}


A

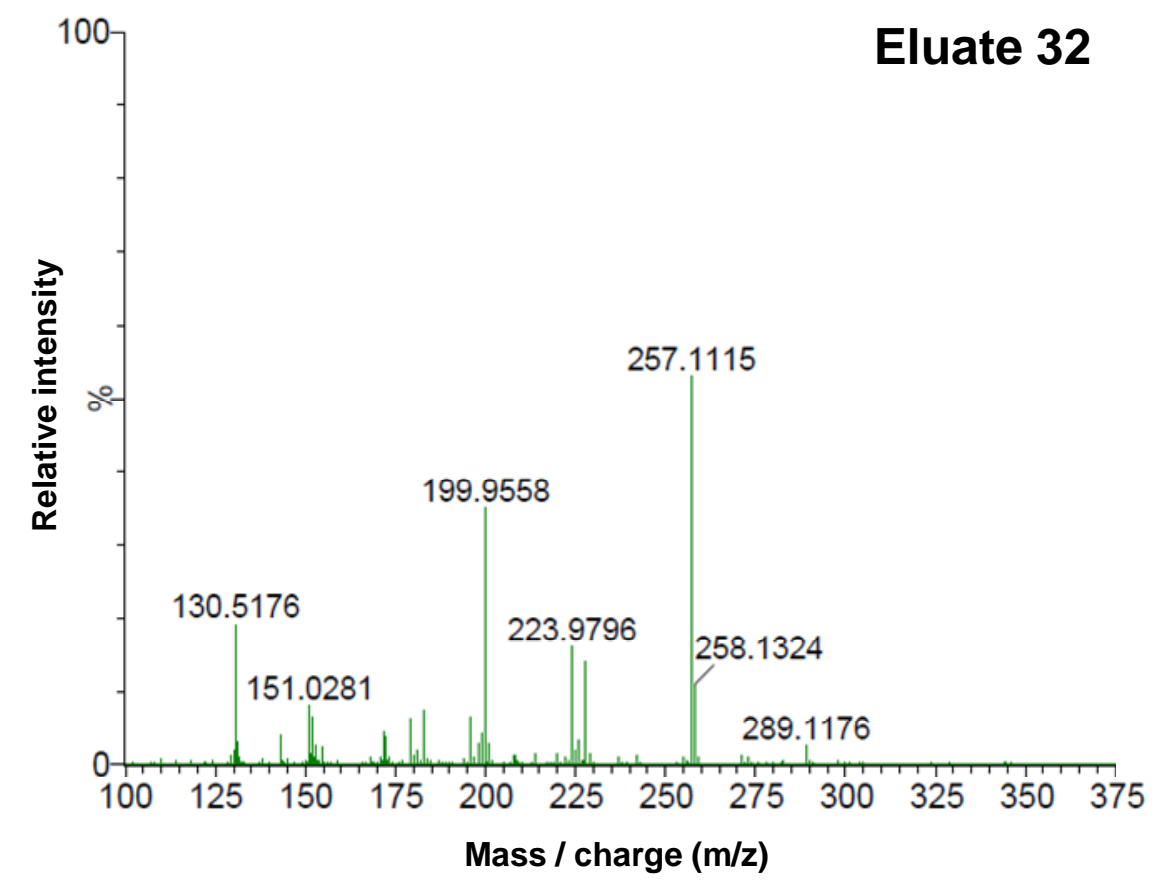

Chapter 3 - Figure 4 
B
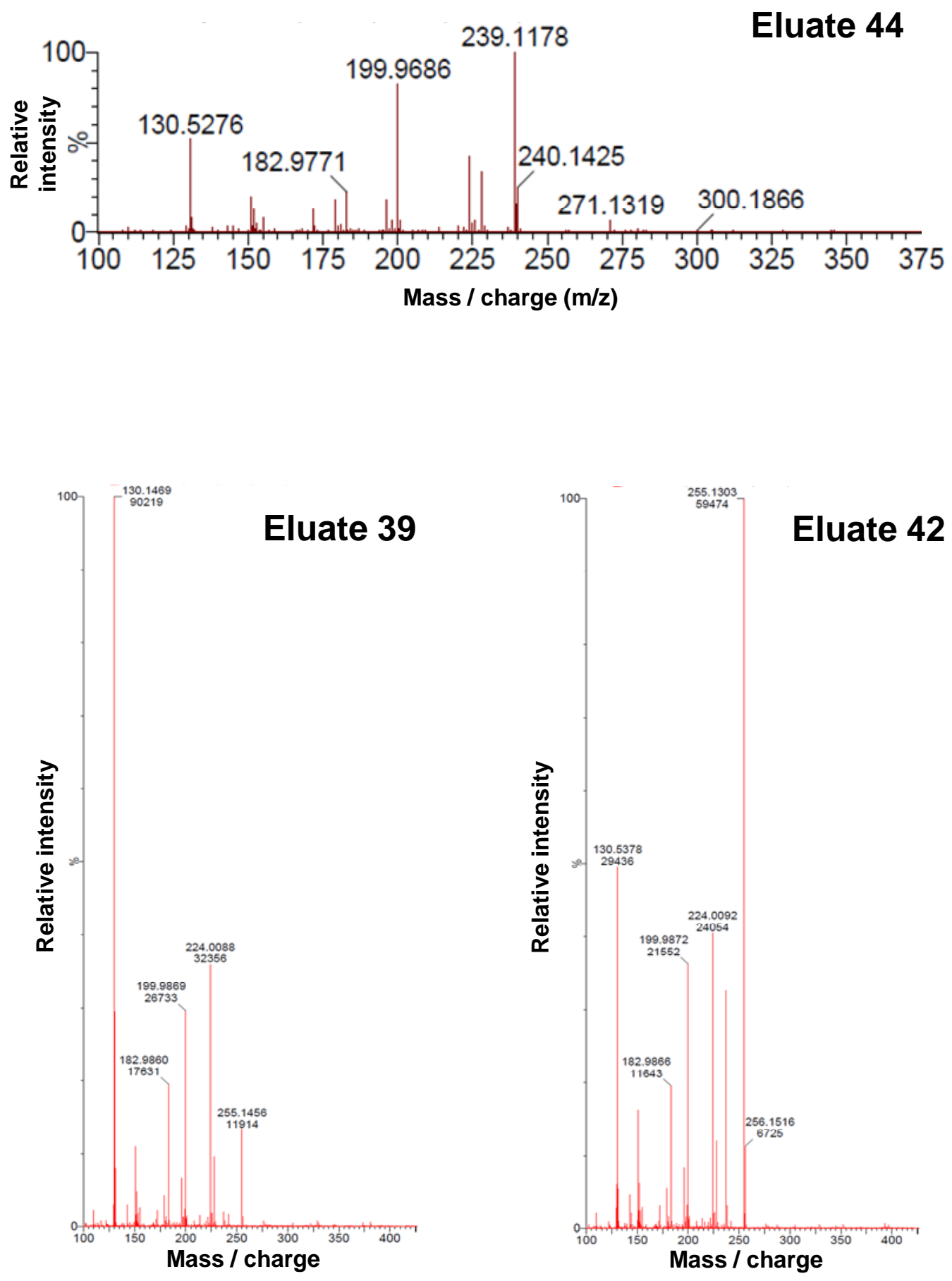

Chapter 3 - Figure 4 
Neotyphodium_lolii Epichloe festucae Claviceps_gigantea Claviceps_africana Claviceps_purpurea_P1 Claviceps_purpurea_20102 Claviceps_fusiformis Aspergillus_fumigatus

Neotyphodium_lolii Epichloe_festucae Claviceps_gigantea Claviceps_africana Claviceps_purpurea_P1 Claviceps_purpurea_20102 Claviceps_fusiformis Aspergillus_fumigatus

Neotyphodium_lolii Epichloe_festucae Claviceps_gigantea Claviceps_africana Claviceps_purpurea_P1 Claviceps_purpurea_20102 Claviceps_fusiformis Aspergillus_fumigatus

Neotyphodium_lolii Epichloe_festucae Claviceps_gigantea Claviceps_africana Claviceps_purpurea_P1 Claviceps_purpurea_20102 Claviceps_fusiformis Aspergillus_fumigatus

Neotyphodium_lolii Epichloe_festucae Claviceps_gigantea Claviceps africana Claviceps_purpurea_P1 Claviceps_purpurea_20102 Claviceps_fusiformis Aspergillus_fumigatus

Neotyphodium_lolii Epichloe_festucae Claviceps_gigantea Claviceps_africana Claviceps_purpurea_P1 Claviceps_purpurea_20102 Claviceps_fusiformis Aspergillus_fumigatus

Neotyphodium_lolii Epichloe_festucae Claviceps_gigantea Claviceps_africana Claviceps_purpurea_P1 Claviceps_purpurea_20102 Claviceps_fusiformis Asneraillus fumiaatus
HKLVLSPMTRFRADNEGVPLPYVKTYYCQRASLPGTLLLTEATAISRRAR 50 HKLVLSPMTRFRADNEGVPLPYVKTYYCQRASLPGTLLLTEATAISRRAR 50 HKIVLSPMTRIRADDHGVPLPYVKTYYAQRACVRGTLLITEAVAVCPRAK 50 HKIVLSPMTRIRADDDGVPLPCVQTYYAQRASVRGTLLITEAVAICPRAK 50 HKIVLSPMTRFRADDNGVPLSYMKTFYAQRASVRGTLLVTDAVAICPRTK 50 HKIVLSPMTRFRADDNGVPLSYMKTFYAQRASVRGTLLVTDAVAICPRTK 50 HKVVLSPMTRFRADNDGVPLSYVKSYYGQRASIRGTLLITEAVAICPRAK 50 HRMIMAPTTRFRADGQGVPLPFVQEYYGQRASVPGTLLITEATDITPKAM 50

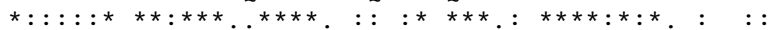

GFPNVPGIWSQEQIAGWKEVVDAVHAKGSYIWLQLWATGRAAEVGVLKAN 100 GFPNVPGIWSQEQIAGWKEVVDAVHAKGSYIWLQLWATGRAAEVGVLKAN 100 GLPNIPGIWSDSQIAAWKDVVDEVHSMGCSIWIQLWATGRASEMETLSSL 100 GIPNIPGIWNHDQMVAWKEVVDOVHARGCFIWVQLWATGRASEMETLRRN 100 GFPNVPGIWHKDQIAAWKEVVDEVHSKGSFIWLQLWATGRAADLEALTSQ 100 GFPNVPGIWHKDRIAAWKEVVDEVHSKGSFIWLQLWATGRAADLEALTSR 100 GFSNCPGIWHODOIAAWKEVVDEVHSKGSVIWLOLWATGRASDADTLKES 100 GYKHVPGIWSEPQREAWREIVSRVHSKKCFIFCQLWATGRAADPDVLADM 100 ${ }^{*}:{ }^{* * *},:{ }^{*}:::^{*},{ }^{*}: .{ }^{*}: * * * * * * *:: .^{*}$

GFDLVSSSAVPVSPGEPTPRALSDDEINSYIGDFVQAAKNAVLEAGFDGV 150 GFDLVSSSAVPVSPGEPTPRALSDDEINSYIGDFVQAAKNAVLEAGFDGV 150 GFELESSSDVPIASGDPVPRPLSSEDIQLYIREYAQAARNAVHGAGFDGV 150 GFELESSSDVPIAAGDAVPRPLSDQDIQAYIREYAQAARNAVHGAGFDGV 150 GLKLESSSEVPVAPGEPTPRALDEDEIQQYILDYVQGAKNAVHGAGFDGV 150 GLKLGSSSEVPVAPGEPTPRALDEDEIQQYILDYVQGAKNAVHGAGFDGV 150 GFHLESSSDVPVAPGEPVPRPLSEDEIESYIRDYVTGAINAVQGAGFDGI 150 K-DLISSSAVPVEEKGPLPRALTEDEIQQCIADFAQAARNAIN-AGFDGV 148

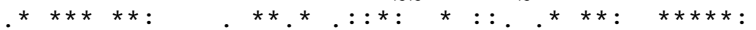

ELHGANGFLIDQFLQSPCNQRTDQWGGCIENRSRFGLEITRRVIDAVGKD 200 ELHGANGFLIDQFLQSPCNQRTDQWGGCIENRSRFGLEITRRVIDAVGKD 200 EIHGAHGYQIDQFLQSSCNRRTDKWGGSIPGRSKFGLDITQSIIDAVGKD 200 EIHGAHGYQVDQFLQSSCNRRTDGWGGSIPARSRFGLDVVRSVIDAVSKD 200 EIHGANGFLIDQFLQSSCNRRTDQWGGSIENRSRFGLEITRGVVDAVGHD 200 EIHGANGFLIDOFLOSSCNRRTDOWGGSIENRSRFGLEITRGVVDAVGHD 200 EIHGANGFLVDQFLQASCNTRADQWGGSIENRSRFGLEITRRVVDAVGKD 200 EIHGANGYLIDQFTQKSCNHRQDRWGGSIENRARFAVEVTRAVIEAVGAD 198 ${ }^{*}:{ }^{* *}:{ }^{*}::^{* * *} *{ }^{* *} * * * * * *^{*}:^{*},::::::::^{* *}, *$

HVGMKLSTWSTFOGMGTMDDLIPOFEHFIMRLREIGIAYLHLANSRWVEE 250 HVGMKLSTWSTFQGMGTMDDLIPQFEHFIMRLREIGIAYLHLANSRWVEE 250 RVGMKLSPWSTFQGMGTMVDLLPQFQHFITCLREMDIAYLHLTNSRWLQE 250 RVGVKLSPWSTFQGMGTMDDLVPQFQHFISRLREMDVAYLHLTNSRWLQE 250 RVGMKLSPWSTFQGMGTMDDLVPQFEHFITCLREMDIAYLHLANSRWVEE 250 RVGMKLSPWSTFQGMGTMDDLVPQFEHFITCLREMDIAYLHLANSRWVEE 250 RVGVKLSPWSTFQGMGTMDDLVAQFEHFISRLREMDIAYIHLVNTRWLEE 250 RVGVKLSPYSQYLGMGTMDELVPQFEYLIAQMRRLDVAYLHLANSRWLDE 248 $:^{* *}:{ }^{* *} .:^{*}:{ }^{* * * * *}:{ }^{*}:{ }^{* *}:::^{*}:{ }^{*} .::^{* *}:{ }^{* *} .{ }^{*}:^{* *}::^{*}$

EDPTIRTHPDIHNETFVRMWGKEKPVLLAGGYGPESAKLVVDETYSD-HK 299 EDPTIRTHPDIHNETFVRMWGKEKPVLLAGGYGPESAKLVVDETYSD-HK 299 EOPGVKIHPDVNNATFVRMWGLEKPIVLAGGYDADSAKRLVDETYSE-HN 299 EQPGIKIHPDVHNATFVRMWGREKPIVLAGGYDADSAKRLVDETYSD-QN 299 EDPSIRTHPDFHNQTFVQMWGKKRPILLAGGYDPDSARRLVDQTYSD-RN 299 EDPSIRTHPDFHNOTFVOMWGKKRPILLAGGYDPDSARRLVDOTYSD-RN 299 EEPGIKTHPDVDNQTFVRMWGNKTPILLAGGYDADSARRLVDETYSD-QN 299 EKP - - - HPDPNHEVFVRVWGQSSPILLAGGYDAASAEKVTEQMAAATYT 294

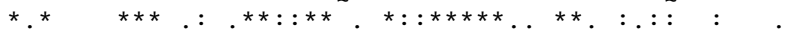

NIGVVFGRHYISNPDLPFRLKMGLPLQKYNRET 332 NIGVVFGRHYISNPDLPFRLKMGLPLQKYNRET 332 NVLVAFGRHYISNPDLPFRLKMGIALOKYNRDT 332 NVLVAFGRHYISNPDLPFRLKMGIALQKYNRDT 332 NVLVVFGRHYISNPDLPFRLRMGIACRSTIETH 332 NVLVVFGRHYISNPDLPFRLRMGIALOKYNRDT 332 NIMVVFGRHYISNPDLPFRLRLGIPLQKYNRDT 332 NVAIAFGRYFISTPDLPFRVMAGIOLOKYDRAS 327

\section{Chapter 3 - Figure 5}


A

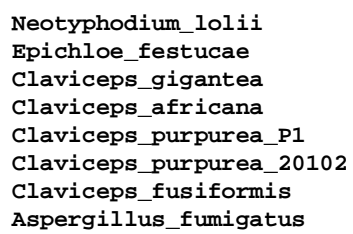

B

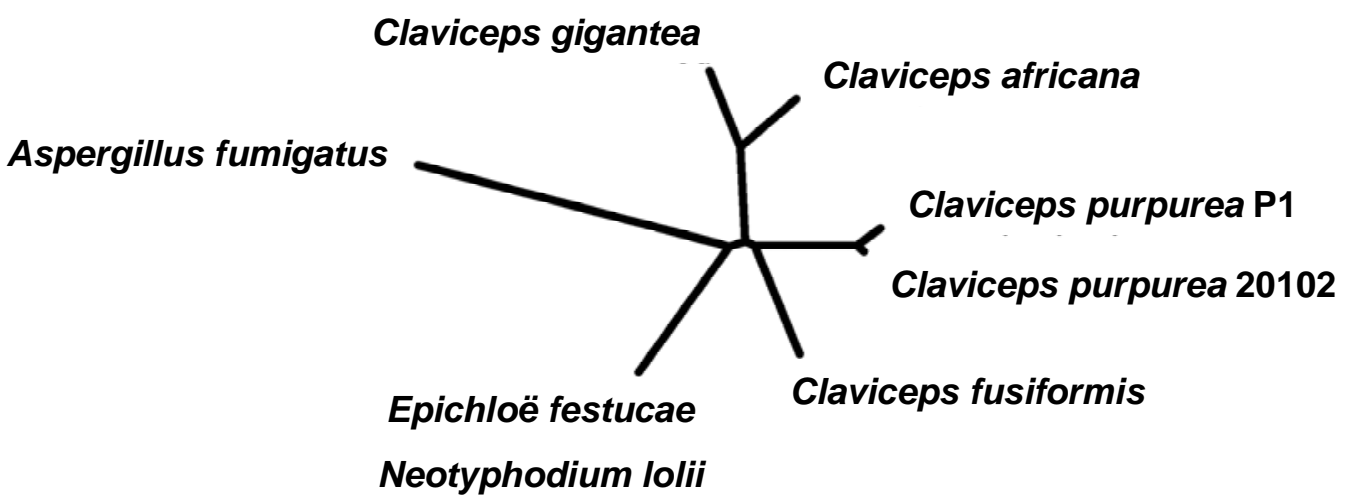

Distance $=0.1$

Chapter 3 - Figure 6 


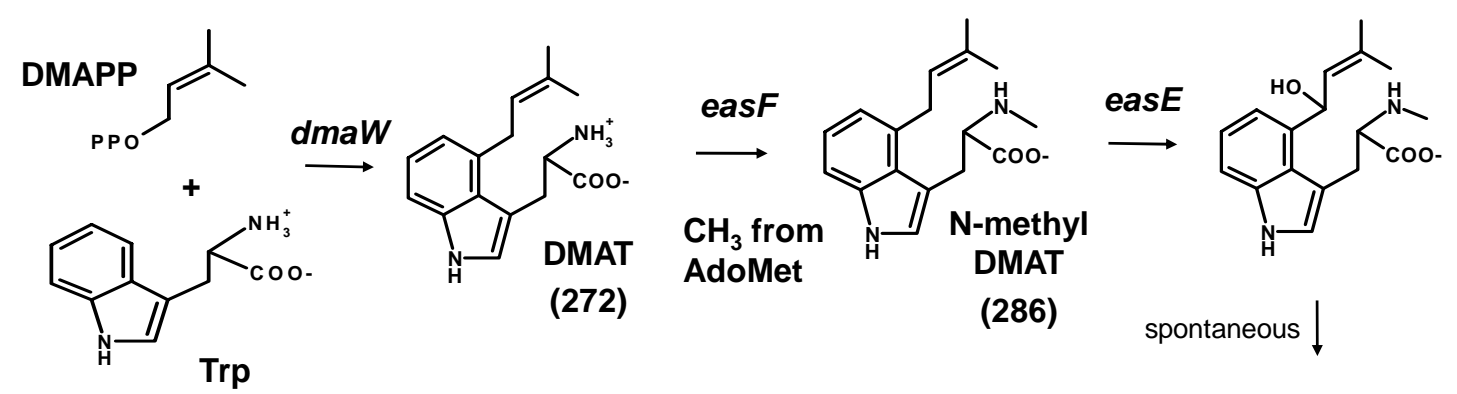

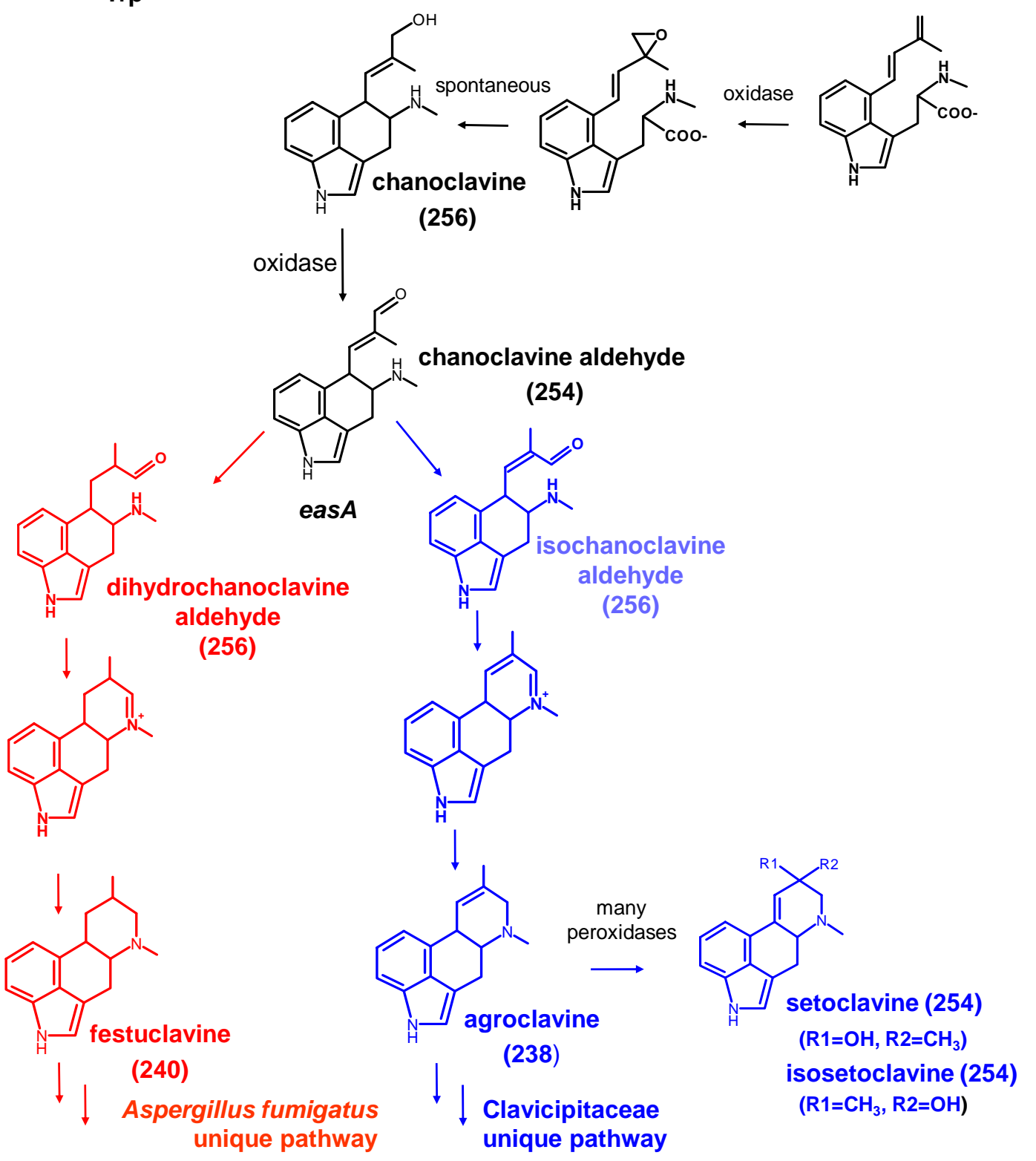

\section{Chapter 3 - Figure 7}




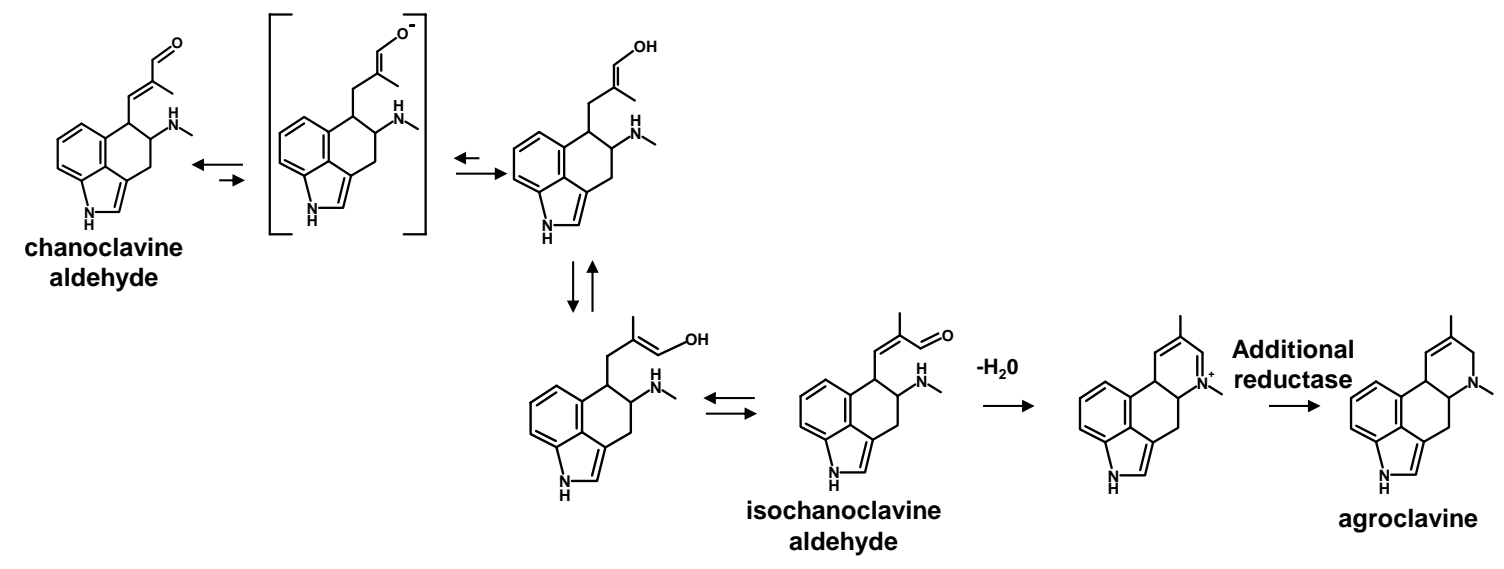

\section{Chapter 3 - Figure 8}




\title{
CHAPTER 4: Association of ergot alkaloids with conidiation in Aspergillus fumigatus
}

Published as: Coyle, C. M., S. C. Kenaley, W. R. Rittenour, and D. G. Panaccione. 2007. Mycologia 99:804-811.

\begin{abstract}
Ergot alkaloids are mycotoxins that affect the nervous and reproductive systems of exposed individuals through interactions with monoamine receptors. They have been more widely studied in ergot fungi and grass endophytes but also are found in Aspergillus fumigatus, an opportunistic human pathogen that reproduces and disseminates exclusively through conidia. The ergot alkaloids festuclavine and fumigaclavines A, B, and $\mathrm{C}$ are present in or on conidia of $A$. fumigatus. Cultures of the fungus that are free of conidia are difficult to obtain, obscuring comparisons of conidia versus vegetative hyphae as sources of the ergot alkaloids. To create conidiation-deficient strains of A.fumigatus, we manipulated the bristle A gene $(\mathrm{brlA})$, which is a regulatory gene controlling vesicle formation or budding growth necessary for conidiation in Aspergillus spp. Disruption of brlA in A. fumigatus, via homologous recombination, resulted in a non-conidiating mutant that produced bristle-like structures instead of conidiophores and conidia. Moreover, the disrupted strain failed to produce ergot alkaloids as verified by HPLC analyses. Complementation with a wild-type allele restored conidiation and ergot alkaloid production. These results suggest that ergot alkaloids are not produced within the vegetative mycelium of the fungus and are directly associated with conidiation.
\end{abstract}

Key words: aspergillosis, bristle A gene, clavines, mycotoxins. 


\section{INTRODUCTION}

Ergot alkaloids are a complex family of indole-derived secondary metabolites produced by several fungi $(15,17,23,30)$. The ecological roles of ergot alkaloids are poorly understood; however, they have activities against mammals, insects, nematodes, and bacteria $(9,10,23,24,31)$. Their effects on humans are thought to be mediated by their agonistic and/or antagonistic interactions with monoamine neurotransmitter receptors $(17,27,28)$.

Aspergillus fumigatus Fres., a common saprophyte and opportunistic pathogen, produces several ergot alkaloids, including festuclavine and fumigaclavines $\mathrm{A}, \mathrm{B}$, and $\mathrm{C}$ $(13,34,37)$. We recently reported a high-performance liquid chromatography (HPLC) procedure for identification and quantification of these alkaloids, and demonstrated them to be present in or on conidia of A. fumigatus in quantities that collectively total approximately $1 \%$ of the dry mass of the conidium (25). Fumigaclavine $\mathrm{C}$ is the end product of the A.fumigatus pathway, with festuclavine, fumigaclavine $\mathrm{B}$, and fumigaclavine A (in that sequence) acting as the final three intermediates in its biosynthesis $(23,30,36)$. Presumably, differences in abundance and activity of the enzymes responsible for converting one intermediate to the next account for the accumulation of festuclavine and fumigaclavine A to relatively high concentrations whereas fumigaclavine B is typically present in much lower concentrations $(23,25)$.

In vitro, A. fumigatus sporulates prolifically and cultures free of conidia are very difficult to obtain. Our unpublished observations of ergot alkaloid yields from cultures of A. fumigatus on agar-based media (primarily conidia and limited vegetative hyphae) 
versus submerged, broth-based media (consisting mainly of hyphae and fewer conidia) suggested an apparent association between conidiation and ergot alkaloids.

Previous research on Aspergillus spp. has associated secondary metabolism and sporulation $(5,7)$. For example, sporulation and sterigmatocystin production have been shown to be regulated by a FadA G $\alpha$ protein-dependent signaling pathway in Aspergillus nidulans Eidam (Winter) (18). More specifically, sterigmatocystin production and conidiation are both negatively regulated by a cAMP-dependent protein kinase catalytic subunit (pkaA) (33).

Conidiation has been studied extensively in A. nidulans. Conidiophore development is contingent on differentiation of the fungus from polarized apical extension to budding growth (12). One gene in particular, $b r l A$, has been determined to control conidiophore development and encodes a nucleic acid binding protein (BrlA) (1, $3,6)$. BrlA is an early protein in a signaling cascade that controls the expression of many genes, including some that encode for other regulatory proteins, by binding to brlA response elements (BREs) within the promoter regions of these genes (8). In A. nidulans, transcription of brlA may initiate at different sites resulting in two overlapping transcription units, $b r l A \alpha$ and $\operatorname{brl} A \beta(29)$. The products of these two forms are functionally interchangeable, translated in the same reading frame, and differ only in that the product of $b r l A \beta$ has an additional 23 amino acids at the amino terminus (29). In this paper, we report mutational analyses of the $b r l A$ gene of $A$. fumigatus to produce nonconidiating mutants for the purpose of examining whether ergot alkaloids are produced during vegetative growth or conidiation. 


\section{MATERIALS AND METHODS}

Fungi and culture conditions. Aspergillus fumigatus isolate FGSC A1141 (25) and derivatives were routinely cultured on potato dextrose agar (PDA) prepared from dehydrated potato flakes (Pillsbury, Minneapolis, MN)(20 g/L), D-glucose (20 g/L), and agar $(15 \mathrm{~g} / \mathrm{L})$. Cultures intended for preparation of protoplasts or DNA were grown overnight in $15 \mathrm{~mL}$ of malt extract medium $(50 \mathrm{~g}$ malt extract, $50 \mathrm{~g}$ lactose, $5 \mathrm{~g}$ asparagine, and $0.4 \mathrm{~g} \mathrm{~K}_{2} \mathrm{HPO}_{4}$ per $\mathrm{L}$ ) in a deep Petri dish (20-mm depth, 100-mm diameter; Fisher Scientific, Pittsburgh, PA) on an orbital shaker set at $37^{\circ} \mathrm{C}$ and $150 \mathrm{rpm}$. Non-conidiating mutants were cultured similarly except pieces of agar-based cultures containing hyphal fragments were used in place of conidia as inoculum.

Disruption and complementation of brlA. The presence of a $\mathrm{brlA}$ orthologue in the $A$. fumigatus genome (21) was determined by tblastn search with protein sequence deduced from A. nidulans brlA (1) as a query sequence. A 640-bp fragment internal to the coding sequence of the putative $b r l A$ orthologue was amplified by PCR primed with oligonucleotides brlAF (5'-GCCACGCTTACGACAGAGGT-3') and brlAR (5'CGGTAGATAGGCGACTGGTG -3'). The $50 \mu \mathrm{L}$ PCR reaction contained $50 \mathrm{mM} \mathrm{KCl}$, $10 \mathrm{mM}$ Tris- $\mathrm{HCl}$ (pH 9.0), 0.1\% (v/v) Triton X-100, $1.5 \mathrm{mM} \mathrm{MgCl}_{2}, 200 \mu \mathrm{M}$ of each deoxyribonucleotide triphosphate, $1 \mu \mathrm{M}$ of each primer, and 2.5 units of Taq DNA polymerase (Promega, Madison, Wisconsin), which was added once the thermocycler reached $95^{\circ} \mathrm{C}$ in the initial denaturing period. The reaction began with an initial denaturing step of $2 \min 30 \mathrm{sec}$ at $95^{\circ} \mathrm{C}$, followed by 35 cycles of 1 min at $94{ }^{\circ} \mathrm{C}, 1 \mathrm{~min}$ at $57{ }^{\circ} \mathrm{C}$, and $1 \mathrm{~min}$ at $72{ }^{\circ} \mathrm{C}$, with a final extension step of $72{ }^{\circ} \mathrm{C}$ for $7 \mathrm{~min}$. A $4.6-\mathrm{kb}$ disruption construct, pBRLA1, was generated by ligating the PCR product into the T/A 
overlap vector pCR2.1 (Invitrogen, Carlsbad, California). The construct was linearized at the unique NdeI site located within the $b r l A$ fragment prior to transformation of the fungus. Protoplasts were prepared and transformed according to the protocol described by Murray et al. (20) and with modifications noted previously (14). Protoplasts were cotransformed with the linearized disruption construct, pBRLA1, and a hygromycin resistance plasmid, pMOcosX (22), linearized at a unique NotI site (14). Non-conidiating transformants were identified by their altered colony morphology and color, and were confirmed by light microscopy. Non-conidiating mutants were purified to nuclear homogeneity through repetitive sub-culturing from hyphal tips (since nuclear purification via single conidium isolation was not possible). Homologous recombination between pBRLA1 and the native brlA locus within the transformants was verified by three PCR assays similar to the PCR reaction described above but with different primers and annealing temperatures. The 5 ' border of the recombination event was confirmed by PCR (annealing temperature at $57^{\circ} \mathrm{C}$ ) primed with oligonucleotides UF $\left(5^{\prime}-\right.$ TGTAAAACGACGGCCAGTGAAT-3', which anneals to vector sequences near the universal primer annealing site in pCR2.1) and brlAFscrn (5'CTCCAACGAATGTCCGTCTATG -3', complementary to brlA sequences near the 5'end of the $b r l A$ gene and flanking the intended site of integration) (Fig. 1A). The juxtaposition of sequences near the 3 ' border of the integration event was verified by PCR (annealing temp $55^{\circ} \mathrm{C}$ ) primed from oligonucleotides UR (5'-

AGCTATGACCATGATTACGCCA-3', complementary to vector sequences near the reverse primer annealing site of pCR2.1), and brlARscrn (5'TAGTGACAAGCTCTGCTTGGA -3', which anneals to brlA sequences near the 3'-end 
of the gene and flanking the intended site of integration) (Fig. 1A). PCR across the entire brlA locus was conducted by priming with oligonucleotides brlAFscrn and brlARscrn (both described above) at an annealing temperature of $55^{\circ} \mathrm{C}$.

Integration of the transformation construct also was analyzed by Southern hybridization as described previously (14), except for substitution of a $b r l A$-specific probe. The $b r l A$ probe was made by amplifying wild-type $A$. fumigatus genomic DNA via PCR from primers brlAF and brlAR, according to the PCR conditions described above, substituting $1 \times$ DIG DNA Labeling Mix (Roche, Mannheim, Germany) for unlabeled dNTPs, and increasing the annealing temperature to $57^{\circ} \mathrm{C}$.

The brlA ko strain was complemented with a $2.9-\mathrm{kb}$ fragment containing a fulllength copy of the brlA coding sequences along with approximately $1 \mathrm{~kb}$ of 5 '-flanking sequences and $0.6 \mathrm{~kb}$ of 3 '-flanking sequences. The complementing fragment was amplified by PCR primed from WbrlAF (5'CTCTCCAGGAACCTTGAAACAGGTTCTC-3') and WbrlAR (5'CCACATAGGACACTCGAGGATGGAGACA-3') in a $50 \mu \mathrm{L}$ PCR reaction containing $15 \mathrm{mM}\left(\mathrm{NH}_{4}\right)_{2} \mathrm{SO}_{4}, 50 \mathrm{mM}$ Tris- $\mathrm{HCl}(\mathrm{pH} 9.0), 0.1 \%$ (v/v) Triton X-100, $1.5 \mathrm{mM} \mathrm{MgCl}_{2}$, $200 \mu \mathrm{M}$ of each dNTP, $1 \mu \mathrm{M}$ of each primer, and 1 unit of DyNAzyme DNA polymerase (New England Biolabs, Beverly, MA) added during the initial denaturing step. The thermocycler program was as described above; however, the annealing temperature was $62{ }^{\circ} \mathrm{C}$ and the extension step was for $3 \mathrm{~min} 20 \mathrm{sec}$. The PCR product was cotransformed into a $b r l A$-disrupted mutant along with a phleomycin resistance plasmid, pBC-phleo (Fungal Genetics Stock Center, University of Missouri-Kansas City, Kansas City, MO). Transformants were selected on complete regeneration medium (26) containing 100 
$\mu \mathrm{g} / \mathrm{mL}$ phleomycin and incubated at $37^{\circ} \mathrm{C}$. Complemented transformants were cultured from individual germinated conidia in order to achieve a homokaryotic culture. To test for the presence of the original disruption construct, PCR reactions were initiated from primers brlAFscrn and UF (for the 5 ' flank of the integration) and UR and brlARscrn (for the 3' flank). An additional PCR screen was performed with primers WbrlAF and WbrlaR (described above), to test for the presence of the introduced wild-type allele in the complemented transformants. PCR conditions are provided above with descriptions of the primers (above).

Analysis of ergot alkaloids and conidiation. For routine analysis of ergot alkaloids, small cubes of cultures grown on PDA (containing approximately $8 \mathrm{~mm} \times 8$ $\mathrm{mm}$ of colony surface area) were submerged in $0.5 \mathrm{~mL}$ of $80 \%$ methanol in a $1.9-\mathrm{mL}$ microcentrifuge tube and pulverized with a plastic pestle. The samples were rotated, end over end (44 rpm), for $2 \mathrm{hr}$, and spin-filtered through a $0.2-\mu \mathrm{M}$ pore nylon filter. Ergot alkaloids were detected and quantified from $20 \mu \mathrm{L}$ of the prepared sample by reversephase HPLC on a C18 column with fluorescence detection based on excitation and emission wavelengths of $272 \mathrm{~nm}$ and $372 \mathrm{~nm}$, respectively (25). The identities of peaks corresponding to festuclavine, and fumigaclavines $\mathrm{A}, \mathrm{B}$, and $\mathrm{C}$ were previously established by mass spectral analyses of native and de-esterified fractions (25) and confirmed by elimination of these peaks from extracts of strains in which the first gene in the ergot alkaloid pathway ( $d m a W$ ) was inactivated by gene disruption (14).

To quantify ergot alkaloids and conidiation from the same samples, cultures were gown on PDA for 5 days at $37^{\circ} \mathrm{C}$ and then an additional 9 days at room temperature. Samples containing $50.3 \mathrm{~mm}^{2}$ of colony surface area were extracted from cultures with 
the broader end of a disposable 1-mL pipette tip (Fisher Scientific, catalog number 02681-172). Samples were submerged in $1 \mathrm{~mL} 80 \%$ methanol, agitated by vortexing, and rotated at $44 \mathrm{rpm}$ for $2 \mathrm{hr}$. Separate aliquots were removed for HPLC analyses and for quantification of conidia. Concentrations of ergot alkaloids were based on peak area relative to an external standard of agroclavine (Sigma, St. Louis, MO) as previously described (25). Conidia were enumerated in dilutions of the methanol extracts with a hemacytometer and light microscope. Six samples were analyzed for each treatment. Data from wild type and a complemented brlA disruptant were compared by Student's ttest.

\section{RESULTS}

Disruption and complementation of brlA. The $A$. fumigatus genome contains an orthologue of brlA (GenBank accession XM_747933). The sequence located on chromosome 1 between nucleotides 2258000 and 2259280 was predicted to encode a protein that is $68 \%$ identical to BrlA $\alpha$ from A. nidulans (19). Based on sequence analysis, there is no evidence for capacity to encode the alternate $\operatorname{BrlA} \beta$ product that has been observed in $A$. nidulans (29). BrlA $\beta$ of $A$. nidulans is transcribed from the same locus as $\operatorname{BrlA} \alpha$ and is identical to $\operatorname{BrlA} \alpha$ except for containing an additional 23 amino acids at the amino terminus. In A. fumigatus only 2 of 19 amino acid residues preceding the initiator methionine of $\mathrm{BrlA} \alpha$ are identical to those in the analogous positions of BrlA $\beta$ of $A$. nidulans. A fragment of $A$. fumigatus DNA corresponding to nucleotides 191 to 830 of the BrlA $\alpha$ coding sequence of $A$. nidulans was used to direct homologous recombination of the gene disruption construct pBRLA1 at the $\operatorname{brlA}$ locus of $A$. fumigatus 
(Fig. 1A). Homologous recombination of pBRLA1 at brlA would interrupt coding sequences common to both $b r l A \alpha$ and brlA $\beta$ (if it were to occur) in A. fumigatus.

Cotransformation of pBRLA1 and NotI-linearized pMOcosX into A. fumigatus resulted in five non-sporulating colonies out of a total of 93 hygromycin-resistant transformants. Agar-based cultures of the putative $b r l A$ disruption strains were white and fluffy, unlike the dense, sooty, dark-green appearance of wild-type cultures (Fig. 2A). Observed by light microscopy, the non-conidiating cultures had extensive, bristle-like, aerial hyphae that lacked the swollen apical vesicles, phialides, and conidia of mature conidiophores (Fig. 2B). Putative disruptants that had been sub-cultured from hyphal tips six times in an effort to achieve a homokaryotic culture were analyzed by PCR and Southern blot hybridization. Amplification of transformant DNA annealed to primer sets UF and brlAFscrn resulted in an 899-bp product (Fig. 1). Similarly, PCR from UR and brlARscrn produced an 863-bp product. Products of these sizes from transformants but not from the wild type indicated integration of the disruption construct into the native copy of brlA (Fig. 1). The lack of a PCR product from these transformants when amplification was primed from brlAFscrn and brlARscrn indicated the absence of a wildtype copy of $b r l A$. Integration of one or more copies of pBRLA1 at brlA results in a large distance between priming sites, whereas in the wild type the primer annealing sites are separated by only $910 \mathrm{bp}$ (Fig. 1). Moreover, the absence of a product in the $\mathrm{brlA}$ disrupted strains (Fig. 1B) indicated nuclear homogeneity of these strains, since any wildtype nuclei in the colony would provide template for amplification of the 910-bp product. Southern blot analyses confirmed the presence of multiple disruption constructs within brlA, which is evident by a large shift in the size of the AccI fragment containing the 
targeted gene region (Fig. 1C). The lack of multiple bands hybridizing to transformant brlA ko17 on the Southern blot verified that random integration of constructs elsewhere in the genome had not occurred (Fig. 1C).

Disrupted strain brlA ko17 was cotransformed with a 2.9-kb PCR product containing the wild-type brlA allele and NotI-linearized pBCphleo. Six conidiating, phleomycin-resistant transformants were analyzed by PCR. The presence of a wild-type copy of brlA in the conidiating transformants was demonstrated by the presence of a 2.9$\mathrm{kb}$ product in reactions primed with WbrlAF and WbrlAR (Fig. 3). Additional PCR analyses of the brlA locus in the complemented strains indicated that pBRLA1 was still present at the previously disrupted $\operatorname{brl} A$ locus, since products of the predicted size were amplified from primer pairs flanking the site of integration of pBRLA1 (Fig. 3). Thus the complementing wild-type $b r l A$ fragment had integrated at a locus other than the native brlA, and the recipient strain was derived from a $b r l A$ disruptant. Similar quantities of conidia were produced by a brlA-complemented disruptant and the wild type (Table 1).

\section{Analysis of ergot alkaloid production in brlA-disrupted mutants and complemented}

derivatives. Mycelia of $b r l A$-disrupted mutants contained no detectable ergot alkaloids

(Fig. 4, Table 1). In contrast, conidiating colonies of wild-type and the brlAcomplemented strain accumulated festuclavine, and fumigaclavines $\mathrm{A}, \mathrm{B}$, and $\mathrm{C}$ in quantities that were similar by strain (Fig. 4, Table 1). The relatively large peak eluting at 53 minutes in the brlA ko17 profile represents a metabolite associated with hyphae. It is present in low levels in wild-type and complemented extracts (Fig. 4), which come from colonies that contain abundant conidiophores and conidia but limited hyphae. Its 
presence in ergot alkaloid-deficient strains derived by knocking out the gene $d m a W(14)$ indicates that it is unrelated to ergot alkaloids.

Examination of the promoter region of ergot alkaloid cluster genes for bristle A response element (BRE) sequences. Since ergot alkaloids were only produced in cultures expressing a functional $\mathrm{brlA}$, the chromosomal region containing 11 genes from eas $A$ to eas $F$ of the ergot alkaloid gene cluster $(14,30)$ was examined for the BRE sequence, 5'-(C/A)(A/G)AGGG(G/A)-3' (8), and its 26 occurrences were mapped (Fig. 5). Given the degenerate nature of the BRE sequence and its ability to function in both orientations, it will occur by chance once in every $1024 \mathrm{bp}$ of random sequence. BREs occurred once every $1404 \mathrm{bp}$ in the coding sequences, whereas in the 5 ' non-coding regions these elements occurred once every 409 bp, making BREs about 3.4 times more common in the $5^{\prime}$ non-coding regions. As assessed in a paired t-test, BREs occurred at a higher frequency per bp in 5' non-coding sequences than in the corresponding coding sequences $(P=0.03)$. However, there were no occurrences of the BRE sequences upstream of three of 11 genes, including $d m a W$, the gene controlling the first step in the ergot alkaloid pathway (Fig. 5). Six other genes had less than three BREs within their upstream non-coding sequences. One 5' non-coding region contained four BRE sequences, and due to divergent orientation of genes, this non-coding sequence was part of the promoter region of two ergot alkaloid cluster genes.

\section{DISCUSSION}

Disruption of brlA in A. fumigatus resulted in a non-conidiating mutant lacking any recognizable conidiophores. A similar phenotype has been reported recently by Mah and Yu (19) for brlA mutants of A. fumigatus constructed by a different knockout strategy. 
The absence of ergot alkaloids in our non-conidiating mutants indicates that ergot alkaloids are not produced during vegetative growth and are solely associated with conidiation in A. fumigatus.

The phenotype of the A. fumigatus brlA mutants indicates that $b r l A$ functions similarly in A. fumigatus as it does in A. nidulans. brlA was first discovered as the Mendelian locus defective in mutants that failed to conidiate but instead grew bristles reaching lengths 20-30 times the normal conidiophore length (11). The wild-type allele was cloned by complementation of the $b r l A$ mutant via transformation and recovery of the transforming DNA (35). Adams et al. (1) demonstrated that expression of brlA under conditions that typically repress conidiation in A. nidulans (submerged broth culture) resulted in production of structures that produced conidium-like cells. Two developmentally regulated genes downstream of $b r l A, a b a A$ and wetA, were not expressed in the nonfunctional brlA mutant but were transcribed in the modified strains expressing $\mathrm{brlA}$. Furthermore, disruption of $\mathrm{Cys}_{2}-\mathrm{His}_{2}-\mathrm{Zn}(\mathrm{II})$ coordination sites resulted in the inability of BrlA to induce the conidiation pathway, thus supporting the functionality of BrlA as a transcription factor that requires two zinc fingers to bind DNA (2).

Links between sporulation and secondary metabolite production in Aspergillus spp. have been demonstrated by mutational analyses of other genes that regulate sporulation $(5,7)$. Relevant studies in A. fumigatus include work done on the regulatory genes stuA (32) and laeA (4). Strains mutated at stuA produced conidiophores that were stunted, and appeared as vesicles that lacked phialides and arose directly from septate hyphae (32). In microarray analysis, stuA mutants showed changes in expression levels 
of genes in two separate gene clusters that are hypothesized to control production of unidentified secondary metabolites. Numerous other genes were affected, including those encoding and proteins involved in development and morphogenesis, such as the cell wall protein, PhiA, and a sexual development repressor protein, Pro1. Mutation of laeA impaired conidiophore (and conidium) development in broth cultures of A. fumigatus but laeA mutants conidiated normally on agar-based media. LaeA regulates expression of genes involved in conidiophore and rodlet formation as well as genes involved in production of secondary metabolites such as gliotoxin (4).

Whether BrlA directly regulates the ergot cluster genes or whether those genes are controlled by a factor or factors whose expression depends upon brlA has not been established. Over all, BREs were more abundant in the 5' non-coding sequences compared to their corresponding coding sequence. However, the brlA response element was absent from the 5' non-coding sequences of three ergot alkaloid cluster genes (including $\mathrm{dmaW}$ ) and was present in only one or two copies upstream of six other genes. Chang and Timberlake (8) found that at least three BREs must be present in the upstream region to increase transcript accumulation from that gene. The occasional absence and relatively low copy number of the BRE associated with ergot cluster genes indicates that factors controlled by or in addition to BrlA regulate the ergot cluster genes. Previous work demonstrates that among the genes controlled by BrlA are genes encoding other regulatory factors such as AbaA and WetA $(1,6)$. A global analysis of gene expression in a brlA mutant compared to wild type, revealing the genes and secondary metabolite gene clusters controlled by $b r l A$, is already in progress (D.C. Sheppard, McGill University, personal communication). 
Association of ergot alkaloids with conidiation may provide insight into the ecological significance and utility of these toxins to the fungus. When A. fumigatus grows pathogenically, its hyphae colonize host tissue, and conidia are not produced (16). Because ergot alkaloids are not produced in hyphae, it is unlikely that they contribute to the invasive phase of aspergillosis. Conidia are produced during saprophytic growth and serve primarily for dissemination. Due to their exceptionally small size and low specific gravity (25), conidia can be inhaled deeply into the lungs of humans and animals. To initiate infection, conidia must survive the initial host-defense response. The possibility that ergot alkaloids contribute to increasing the survival of inhaled conidia remains to be investigated.

\section{ACKNOWLEDGEMENTS}

This article is published with the approval of the Director of the WV Agricultural and Forestry Experiment Station as Scientific Article no. 2990. This project was supported by the National Research Initiative of the USDA Cooperative State Research, Education and Extension Service, grant number 2005-35318-16184. 


\section{REFERENCES}

1. Adams, T. H., M. T. Boylan, and W. E. Timberlake. 1988. brlA is necessary and sufficient to direct conidiophore development in Aspergillus nidulans. Cell 54:353-362.

2. Adams, T. H., H. Deising, and W. E. Timberlake. 1990. brlA requires both zinc fingers to induce development. Mol. Cell Biol. 10:1815-1817.

3. Adams, T. H., J. K. Wieser, and J.-H. Yu. 1998. Asexual Sporulation in Aspergillus nidulans. Microbiol. Mol. Biol. Rev. 62:35-54.

4. Bok, J. W., S. A. Balajee, K. A. Marr, D. Andes, K. F. Nielsen, J. C. Frisvad, and N. P. Keller. 2005. LaeA, a regulator of morphogenetic fungal virulence factors. Eukaryot. Cell 4:1574-1582.

5. Bok, J. W., and N. P. Keller. 2004. LaeA, a regulator of secondary metabolism in Aspergillus spp. Euk. Cell 3:527-535.

6. Boylan, M. T., P. M. Mirabito, C. E. Willett, C. R. Zimmerman, and W. T. Timberlake. 1987. Isolation and physical characterization of three essential conidiation genes from Aspergillus nidulans. Mol. Cell Biol. 7:3113-3118.

7. Calvo, A. M., R. A. Wilson, J. W. Bok, and N. P. Keller. 2002. Relationship between secondary metabolism and fungal development. Microbiol. Mol. Biol. Rev. 66:447-459.

8. Chang, Y. C., and W. E. Timberlake. 1992. Identification of Aspergillus brlA response elements selection in yeast. Genetics 133:29-38. 
9. Clay, K., and G. P. Cheplick. 1989. Effect of ergot alkaloids from fungal endophyte-infected grasses on fall armyworm (Spodoptera frugiperda). J. Chem. Ecol. 15:169-182.

10. Clay, K., and C. Schardl. 2002. Evolutionary origins and ecological consequences of endophyte symbiosis with grasses. Am. Nat. 160:S199-S127.

11. Clutterbuck, A. J. 1969. A mutational analysis of conidial development in Aspergillus nidulans. Genetics 63:317-327.

12. Cole, G. T. 1986. Models of cell differentiation in conidial fungi. Microbiol. Rev. 96: 95-132.

13. Cole, R. J., J. W. Kirksey, J. W. Dorner, D. M. Wilson, J. C. Johnson, A. N. Johnson, D. M. Bedell, J. P. Springer, and K. K. Chexal. 1977. Mycotoxins produced by Aspergillus fumigatus species isolated from molded silage. J. Agric. Food Chem. 25:826-830.

14. Coyle, C. M., and D. G. Panaccione. 2005. An ergot alkaloid biosynthesis gene and clustered hypothetical genes from Aspergillus fumigatus. Appl. Environ. Microbiol. 71:3112-3118.

15. Flieger, M., M. Wurst, and R. Shelby. 1997. Ergot alkaloids -- sources, structures and analytical methods. Folia Microbiol. 42:3-30.

16. Fraser, R. S. 1993. Pulmonary aspergillosis: pathologic and pathogenetic features. Pathol. Annu. 28:231-277.

17. Gröger, D., and H. G. Floss. 1998. Biochemistry of ergot alkaloids achievements and challenges. Alkaloids 50:171-218. 
18. Hicks, J. K., J.-H. Yu, N. P. Keller, and T. H. Adams. 1997. Aspergillus sporulation and mycotoxin production both require inactivation of the FadA G protein-dependent signaling pathway. EMBO J. 16:4916-4923.

19. Mah, J.-H., and J.-H. Yu. 2006. Upstream and downstream regulation of asexual development in Aspergillus fumigatus. Euk. Cell 5:1585-1595.

20. Murray, F. R., G. C. M. Latch, and D. B. Scott. 1992. Surrogate transformation of perennial ryegrass, Lolium perenne, using genetically modified Acremonium endophyte. Mol. Gen. Genet. 233:1-9.

21. Nierman, W. C., A. Pain, M. J. Anderson, and e. al. 2005. Genomic sequence of the pathogenic and allergenic filamentous fungus Aspergillus fumigatus. Nature 438:1151-1156.

22. Orbach, M. J. 1994. A cosmid with a HyR marker for fungal library construction and screening. Gene 150:159-162.

23. Panaccione, D. G. 2005. Origins and significance of ergot alkaloid diversity in fungi. FEMS Microbiol. Lett. 251:9-17.

24. Panaccione, D. G., J. R. Cipoletti, A. B. Sedlock, K. P. Blemings, C. L. Schardl, C. Machado, and G. E. Seidel. 2006. Effects of ergot alkaloids on food preference and satiety in rabbits, as assessed with gene-knockout endophytes in perennial ryegrass (Lolium perenne). J. Agric.Food Chem. 54:4582-4587.

25. Panaccione, D. G., and C. M. Coyle. 2005. Abundant respirable ergot alkaloids from the common airborne fungus Aspergillus fumigatus. Appl. Environ. Microbiol. 71:3106-3111. 
26. Panaccione, D. G., R. D. Johnson, J. Wang, C. A. Young, P. Damrongkool, B. Scott, and C. L. Schardl. 2001. Elimination of ergovaline from a grassNeotyphodium endophyte symbiosis by genetic modification of the endophyte.

Proc. Natl. Acad. Sci. USA 98:12820-12825.

27. Pertz, H. 1996. Naturally occurring clavines: antagonism/partial agonism at 5HT2A receptors and antagonism at $\alpha 1$-adrenoceptors in blood vessels. Planta Med. 62:387-392.

28. Pertz, H., and E. Eich. 1999. Ergot alkaloids and their derivatives as ligands for serotoninergic, dopaminergic, and adrenergic receptors, p. 411-440. In V. Kren and L. Cvak (ed.), Ergot: The Genus Claviceps. Harwood Academic Publishers, The Netherlands.

29. Prade, R. A., and W. E. Timberlake. 1993. The Aspergillus nidulans brlA regulatory locus consists of overlapping transcription units that are individually required for conidiophore development. EMBO J. 12:2439-2447.

30. Schardl, C., D. G. Panaccione, and P. Tudzynski. 2006. Ergot alkaloids-biology and molecular biology, p. 45-86. In G. A. Cordell (ed.), Alkaloids Chem. Biol., vol. 63. Academic Press, San Diego, CA.

31. Schwarz, G., and E. Eich. 1983. Influence of ergot alkaloids on growth of Streptomyces purpurascens and production of its secondary metabolites. Planta Med. 47:212-214.

32. Sheppard, D. C., T. Doedt, L. Y. Chiang, H. S. Kim, D. Chen, W. C. Nierman, and S. G. Filler. 2005. The Aspergillus fumigatus StuA protein 
governs the up-regulation of a discrete transcriptional program during the acquisition of developmental competence. Mol. Biol. Cell 16:5866-5879.

33. Shimizu, K., and N. P. Keller. 2001. Genetic involvement of a cAMPdependent protein kinase in a $\mathrm{G}$ protein signaling pathway regulating morphological and chemical transitions in Aspergillus nidulans. Genetics 157:591-600.

34. Spilsbury, J. F., and S. Wilkinson. 1961. The isolation of festuclavine and two new clavine alkaloids from Aspergillus fumigatus Fres. J. Chem. Soc. 5:20852091.

35. Timberlake, W. E., M. T. Boylan, M. B. Cooley, P. M. Mirabito, E. B. O'Hara, and C. E. Willett. 1985. Rapid identification of mutationcomplementing restriction fragments from Aspergillus nidulans cosmids. Exp. Mycol. 9:351-355.

36. Unsöld, I. A., and S.-M. Li. 2006. Reverse prenyltransferase in the biosynthesis of fumigaclavine $\mathrm{C}$ in Aspergillus fumigatus: gene expression, purification, and characterization of fumigaclavine $C$ synthase. ChemBioChem 7:158-164.

37. Yamano, T., K. Kishino, S. Yamantodani, and M. Abe. 1962. Investigation on ergot alkaloids found in cultures of Aspergillus fumigatus. Takeda Kenkyusho Nempo (Annual Report Takeda Research Laboratories) 21:95-101. 


\section{FIGURE LEGENDS}

Figure 1: Homologous recombination at brlA. (A) Primers brlAF and brlAR prime amplification of a 640-bp internal fragment from $\mathrm{brlA}$. Gene disruption construct pBRLA1 contains the internal fragment of brlA and was linearized at a unique NdeI site prior to transformation. The bottom panel shows integration of three copies of the disruption construct within brlA and the positions of primers used in PCR experiments to

demonstrate integration at this locus. (B) Homologous recombination of the transforming DNA at $b r l A$ was demonstrated by PCR with primer set brlAFscrn and UF confirming integration at the 5'-end of the gene and primer set UR and brlARscrn confirming integration at the $3^{\prime}$-end. Amplification across the 5 ' and 3 ' junctions of integration resulted in 899-bp and 863-bp fragments, respectively. Primer positions are shown near the bottom of part A. The wild-type (wt) screen was a PCR primed from brlAFscrn and brlARscrn, and amplification does not occur from DNA that has disruption constructs within the intended site of integration due to the increase in template length. PCR of non-transformed (wt) DNA gives a product of $910 \mathrm{bp}$. (C) Integration of transforming DNA at the targeted site was confirmed by Southern blot hybridization. Fungal DNA extracts were digested with $A c c$ I and hybridized with a digoxigenin-dUTP labeled brlA probe. The large size of the brlA-containing fragments in strains brlA ko17 and brlA ko40 indicate three or more copies of pBRLA1 are present in the integration site. The relative mobilities of relevant fragments of $B s t$ EII-digested bacteriophage lambda DNA are indicated on the left in panels B and C. 
Figure 2: Morphology of $b r l A$ mutants. (A) Cultures of $A$. fumigatus wild type (right) and $\operatorname{brlA}$ ko (left) on agar-based media. (B) Light microscopy of the same cultures allows for comparisons of wild-type conidiophore development and spore production (right) versus bristle-like structures in $b r l A$ ko (left). Scale bar represents $10 \mu \mathrm{m}$.

Figure 3: PCR analyses of complemented transformant. The presence of an introduced wild-type allele of $\operatorname{brl} A$ in the complemented strain $(b r l A c t)$ was shown by PCR with primer set WbrlAF and WbrlAR. Retention of the disruption construct previously integrated at the original brlA locus was detected by PCR with primers flanking the 5'end of the integration site (brlAFscrn and UF) and primers flanking the 3'-end of the integration site (brlARscrn and UR). Primer positions are shown in Fig. 1. The relative mobilities of relevant fragments of BstEII-digested bacteriophage lambda DNA are indicated to the left of the photograph.

Figure 4: HPLC traces of wild-type A. fumigatus (wt), a brlA disrupted strain (brlA ko17), and a complemented strain of $\operatorname{brlA}$ ko17 (brlA ct), to which a functional copy of brlA has been added. Ergot alkaloids were extracted with $80 \%$ methanol and analyzed by reverse phase HPLC with fluorescence detection (excitation and emission wavelengths of $272 \mathrm{~nm}$ and $372 \mathrm{~nm}$, respectively). $\mathrm{B}=$ fumigaclavine $\mathrm{B} ; \mathrm{F}=$ festuclavine; $\mathrm{A}=$ fumigaclavine $\mathrm{A} ; \mathrm{C}=$ fumigaclavine $\mathrm{C}$.

Figure 5: Occurrence of BRE sequences in the ergot alkaloid gene cluster. This map shows the locations of the BRE sequence within $5^{\prime}$ non-coding regions (13), coding 
regions (9), $3^{\prime}$ non-coding regions (3), and within an intron (1, marked with asterisk) of these genes. 
Table 1. Mean conidiation and ergot alkaloid production by strain $(n=6)$ for the wild-type (wt), the complemented $\operatorname{brlA}$ knockout (brlA ct), and the $\operatorname{brl} A$ knockout (brlA ko17) with associated confidence intervals $(\alpha=0.05)$.

\begin{tabular}{lccccc}
\hline \multirow{2}{*}{ Strain } & Conidia $\mathrm{x}$ & \multicolumn{4}{c}{ Ergot alkaloid concentration (fg per conidium) } \\
\cline { 3 - 6 } & $10^{6} / \mathrm{mm}^{2}$ & Festuclavine & Fumigaclavine & Fumigaclavine & Fumigaclavine \\
& & & $\mathrm{A}$ & $\mathrm{B}$ & $\mathrm{C}$ \\
\hline $\mathrm{wt}$ & $1.3( \pm 0.6)^{\mathrm{a}}$ & $10.2( \pm 2.7)$ & $1.1( \pm 0.3)$ & $0.2( \pm 0.0)$ & $6.7( \pm 1.6)$ \\
$\operatorname{brlA} \mathrm{ct}$ & $0.9( \pm 0.4)$ & $15.2( \pm 6.3)$ & $1.1( \pm 0.5)$ & $0.3( \pm 0.2)$ & $8.8( \pm 4.4)$ \\
$\operatorname{brl} \mathrm{k}$ ko17 & $\mathrm{nd}^{\mathrm{b}}$ & $\mathrm{nd}$ & $\mathrm{nd}$ & $\mathrm{nd}$ & $\mathrm{nd}$ \\
\hline
\end{tabular}

${ }^{a}$ Comparison of wt and brlA ct data within each column of table by t-test indicates no significant difference $(P>0.05)$.

${ }^{\mathrm{b}}$ none detected

\section{Chapter 4 - Table 1}




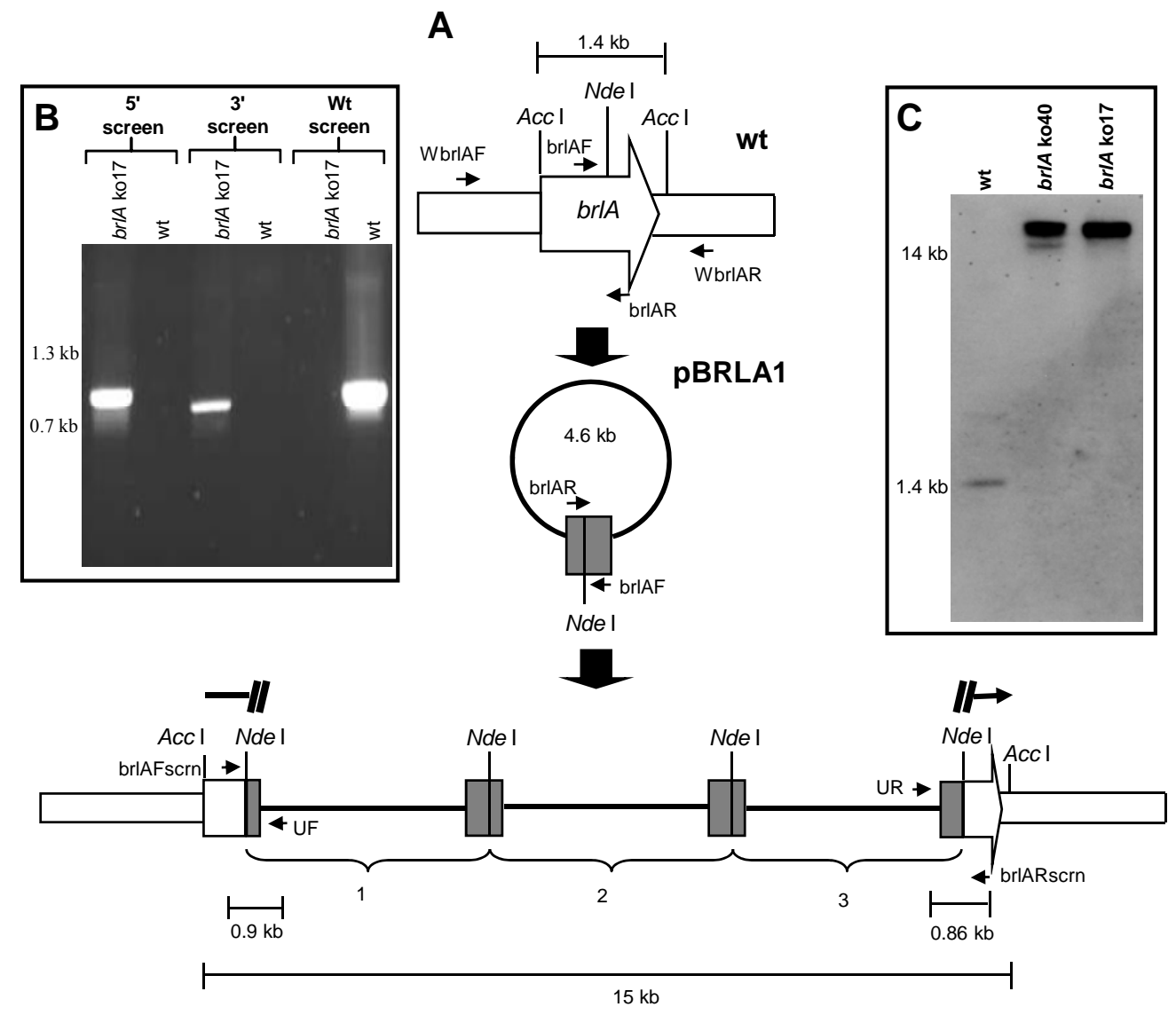

Coyle et al., Fig 1

\section{Chapter 4 - Figure 1}




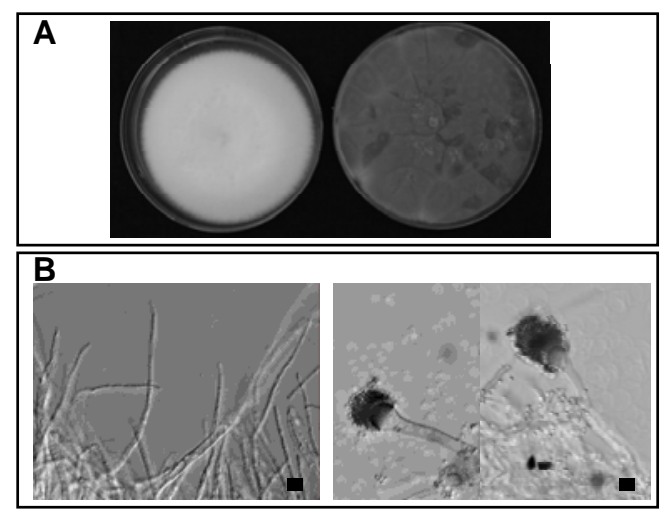

Coyle et al., Fig 2

Chapter 4 - Figure 2 


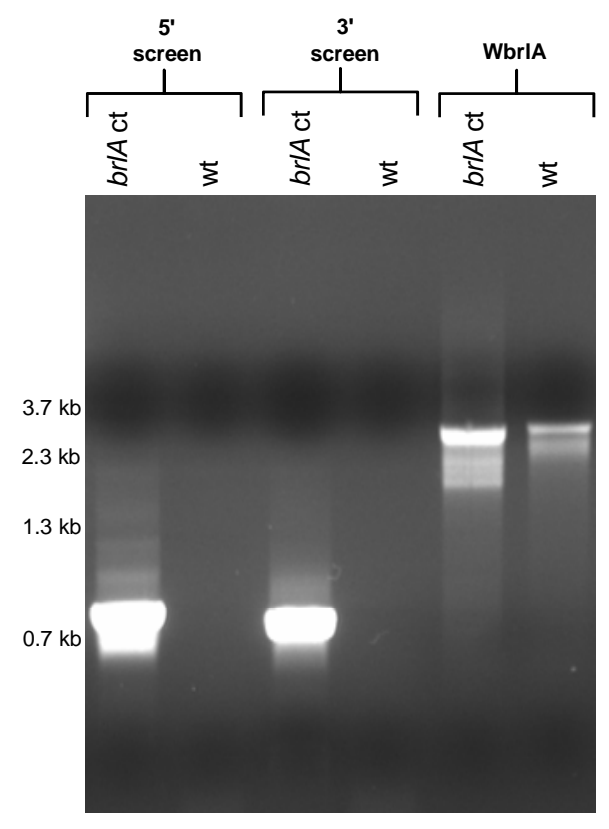

Coyle et al., Fig 3

Chapter 4 - Figure 3 

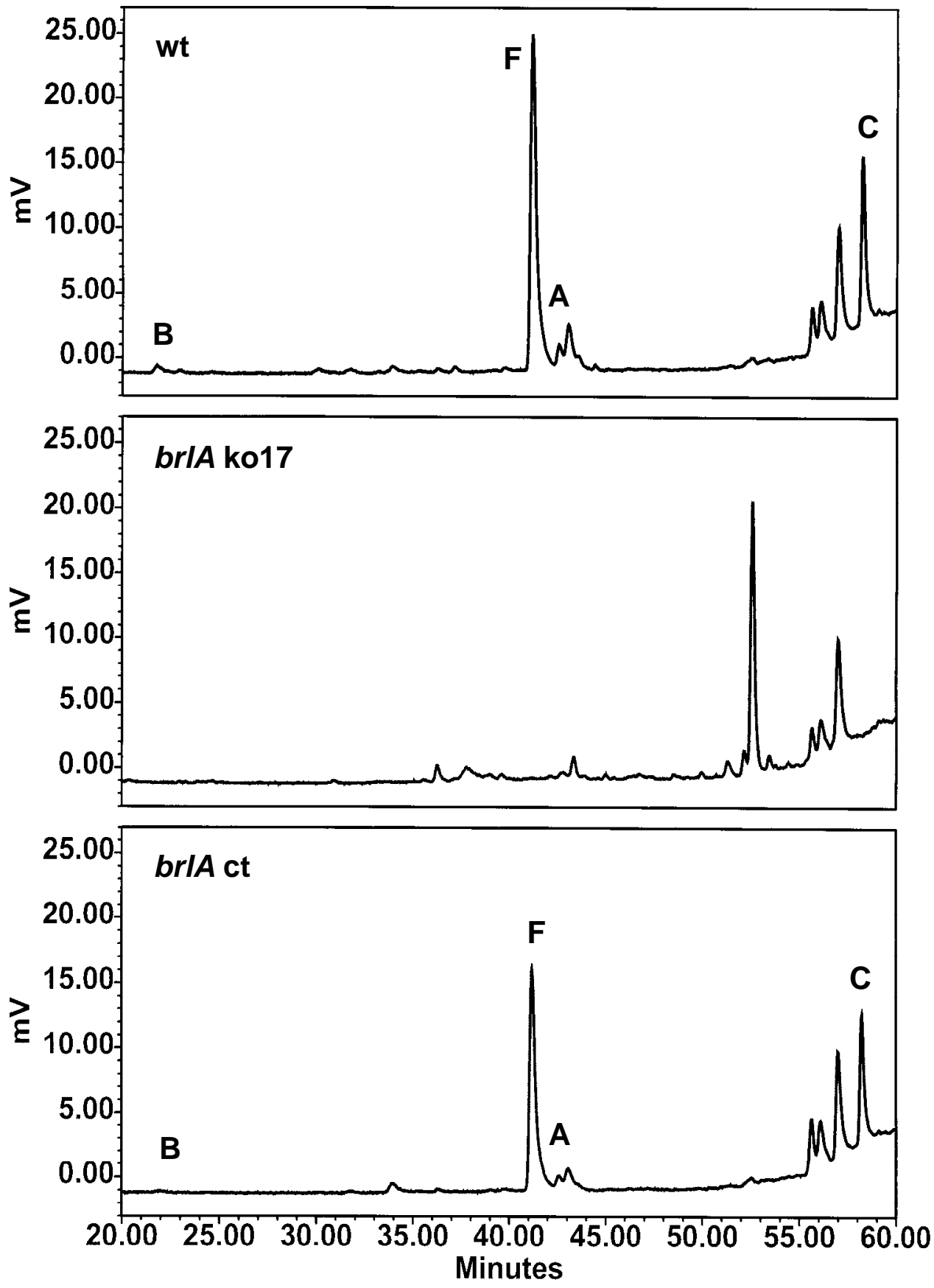

Coyle et al., Fig 4

Chapter 4 - Figure 4 


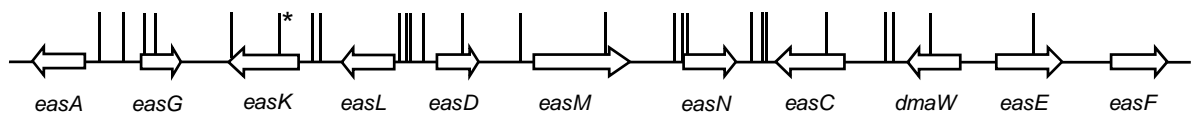

$$
-=\sim 1 \mathrm{~kb}
$$

Coyle et al., Fig 5

\section{Chapter 4 - Figure 5}




\section{SUMMARY AND FUTURE DIRECTIONS}

Ergot alkaloids are mycotoxins capable of interacting with neurotransmitter receptors and causing toxicoses. However, they are also important metabolites that can be medicinally advantageous. The ability to control the spectrum of alkaloids produced is contingent upon understanding how they are synthesized. Aspergillus fumigatus may be a good model system to study ergot alkaloid biosynthesis compared to other ergot alkaloid producers. Therefore this study focuses on ergot alkaloid production in A. fumigatus, examining specifically its association with sporulation and ergot alkaloid synthesis genes common to both A. fumigatus and ergot fungi.

In previous work, Dr. Panaccione had determined that ergot alkaloids were associated with the conidia of $A$. fumigatus. Results of analyses of ergot alkaloid content of spores produced on different substrates indicated that these mycotoxins are present in relatively abundant concentrations regardless of medium. Analysis of the physical properties of conidia of $A$. fumigatus and several other fungi showed that the spores of $A$. fumigatus are significantly smaller, lighter, and of lower specific gravity when compared to those of Aspergillus niger, Aspergillus nidulans, and Stachybotrys chartarum, a fungus of public concern [(5); Appendix 1]. Investigations involving alkaloid accumulation in or on spores prompted a study of whether ergot alkaloids were produced only during sporulation. Knockout of $b r l A$, a gene known to be involved in sporulation in $A$. nidulans, demonstrated this gene to be involved in conidiation in A. fumigatus [(1); Chapter 1]. Lack of a functional BrlA rendered the fungus unable to form proper conidiophores (asexual sporulation structures) and devoid of spores. This mutant also 
lacked ergot alkaloids, thereby indicating that $b r l A$ does affect their production and that the ergot alkaloid pathway and the sporulation pathway are linked, which has also been recently demonstrated with some other secondary metabolites.

The second part of this research focused on understanding genes common to both A. fumigatus and ergot fungi that act early in the ergot alkaloid biosynthesis pathway. Annotation of the genomic region surrounding a homologue of $\mathrm{dmaW}$, the gene controlling the determinant step in the ergot alkaloid pathway as demonstrated previously in studies with Neotyphodium sp. Lp1 (7), revealed the presence of a cluster of genes that potentially could be involved in ergot alkaloid biosynthesis [(2); Chapter 2]. Individual genes in the ergot alkaloid gene cluster and their potential involvement in ergot alkaloid production were investigated in a series of gene knockout experiments. Knockout of the dmaW homologue in A. fumigatus eliminated all ergot alkaloid production in the fungus. This result is consistent with the role DMAT (dimethylallyltryptophan) synthase plays in Neotyphodium sp. Lp1.

Knockout of other clustered genes, eas $A$, eas $E$, and $e a s F$, revealed their involvement in synthesis of these toxins. This research suggested that eas $F$ and easE encode products involved in the second and third steps of the pathway, respectively. The ergot alkaloid profile of easF knockout showed an accumulation of DMAT, due to the mutant's inability to methylate it into N-methyl DMAT (Chapter 2), as well as small accumulations of downstream intermediates, such as festuclavine. These observations imply that the product of eas $F \mathrm{~N}$-methylates DMAT but that ergot alkaloid synthesis can occur very inefficiently even without EasF, given that DMAT synthase can prenylate abrine to form N-methyl DMAT (6). The easE mutant accumulated an intermediate 
characteristically similar to N-methyl DMAT and is presumed to act as an oxidase via adding an hydroxyl group onto this substrate.

Lastly, eas $A$ was determined to encode a putative OYE oxidoreductase, and its product was found to be involved in synthesis of festuclavine from chanoclavine aldehyde in A. fumigatus. The Claviceps purpurea homologue of easA catalyzed synthesis of agroclavine instead of festuclavine, which was evident from complementation of the C. purpurea easA into A. fumigatus easA knockout (Chapter 3). Protein sequence analysis revealed a key difference in a specific amino acid, which is known to be important in other OYEs for reduction of double bonds $(3,4)$. Fungi that produce festuclavine contain a critical active site tyrosine, whereas those that synthesize agroclavine-derived alkaloids typical of ergot fungi (ergopeptines, etc) had phenylalanine at the corresponding position. Therefore, differences in EasA function among homologues have been demonstrated to be responsible for the divergence of the pathway.

This study has demonstrated the coupling of ergot alkaloid production with sporulation. Future research should focus on understanding where expression of these eas genes occurs. Such results may allow us to link intermediates with specific cell types within A. fumigatus. Also future research could focus on analysis of ergot alkaloid production in other sporulation mutants to further characterize the association between ergot alkaloid production and conidiation.

This research also has demonstrated the role of multiple eas genes previously hypothesized to be involved in ergot alkaloid biosynthesis. The genes, eas $F$ and easE, control steps that occur early in the pathway and theoretically should function similarly in ergot fungi. Future research should focus on delineating the roles of other clustered 
genes, such as easD and eas $G$, which are common between $A$. fumigatus and ergot fungi and may share a similar function in both pathways. Additional, unassigned pathway steps that these genes may control include formation of the epoxide following the diene intermediate, chanoclavine aldehyde synthesis, or reduction of the imine, which results from closure of the fourth ring. Further elucidation of the pathway will unveil the involvement of these and other genes within the eas gene cluster.

Since eas $A$ is the point of divergence for the pathway, the discovery of functions of later pathway genes from clavicipitaceous fungi might be discovered by adding candidate genes to the $\mathrm{Cp}$ eas $A$-complemented $A$. fumigatus eas $A$ knockout. Further characterization of the EasA homologues via point mutation analyses are necessary to understand the genetic basis for differences observed in their enzymatic functions. 


\section{REFERENCES}

1. Coyle, C. M., S. C. Kenaley, W. R. Rittenour, and D. G. Panaccione. 2007. Association of ergot alkaloids with conidiation in Aspergillus fumigatus. Mycologia 99:804-811.

2. Coyle, C. M., and D. G. Panaccione. 2005. An ergot alkaloid biosynthesis gene and clustered hypothetical genes from Aspergillus fumigatus. Appl. Environ. Microbiol. 71:3112-3118.

3. Kohli, R. M., and V. Massey. 1998. The oxidative half-reaction of old yellow enzyme. The role of tyrosine 196. J. Biol. Chem. 273:32763-32770.

4. Meah, Y., and V. Massey. 2000. Old Yellow Enzyme: Stepwise reduction of nitro-olefins and catalysis of aci-nitro tautomerization. Proc. Natl. Acad. Sci. USA 97:10733-10738.

5. Panaccione, D. G., and C. M. Coyle. 2005. Abundant respirable ergot alkaloids from the common airborne fungus Aspergillus fumigatus. Appl. Environ. Microbiol. 71:3106-3111.

6. Unsöld, I. A., and S.-M. Li. 2005. Overproduction, purification and characterization of FgaPT2, a dimethylallyltryptophan synthase from Aspergillus fumigatus. Microbiology 151:1499-1505.

7. Wang, J., C. Machado, D. G. Panaccione, H.-F. Tsai, and C. L. Schardl. 2004. The determinant step in ergot alkaloid biosynthesis by an endophyte of perennial ryegrass. Fungal Genet. Biol. 41:189-198. 


\title{
APPENDIX 1: Abundant respirable ergot alkaloids from the common airborne fungus Aspergillus fumigatus
}

Published by: Panaccione, D. G., and C. M. Coyle. 2005. Appl. Environ. Microbiol. 71:3106-3111.

\begin{abstract}
Ergot alkaloids are mycotoxins that interact with several monoamine receptors, negatively affecting cardiovascular, nervous, reproductive, and immune systems of exposed humans and animals. Aspergillus fumigatus, a common airborne fungus and opportunistic human pathogen, can produce ergot alkaloids in broth culture. The objectives of this study were to determine if $A$. fumigatus accumulates ergot alkaloids in respirable form in or on its conidia, to quantify ergot alkaloids associated with conidia produced on several different substrates, and to measure relevant physical properties of those conidia. We found at least four ergot alkaloids - fumigaclavine $\mathrm{C}$, festuclavine, fumigaclavine $\mathrm{A}$, and fumigaclavine $\mathrm{B}$, in order of abundance - associated with conidia of $A$. fumigatus. Under environmentally relevant conditions, the total mass of ergot alkaloids often constituted $>1 \%$ of the mass of the conidium. Ergot alkaloids were extracted from conidia produced on all media tested, with the greatest quantities observed when the fungus was cultured on latex paint or cultured maize seedlings. Physical properties of conidia likely to affect their respirability, i.e. diameter, mass, and specific gravity, were significantly lower in A. fumigatus than in Aspergillus nidulans, Aspergillus niger, and Stachybotrys chartarum. Demonstration of relatively high concentrations of ergot alkaloids associated with conidia of $A$. fumigatus presents opportunities for investigations of potential contributions of the toxins to adverse health effects associated with the fungus and to aspects of the biology of the fungus that contribute to its success.
\end{abstract}




\section{INTRODUCTION}

Ergot alkaloids are a complex family of indole-derived alkaloids that have a long history of association with human suffering. The contamination of rye and other grain crops with alkaloid-rich sclerotia of the ergot fungus Claviceps purpurea was responsible for gangrenous and convulsive forms of ergotism known as St. Anthony's fire or holy fire (19). Other ergot alkaloid-producing fungi such as the closely related Neotyphodium spp. endophytes of forage grasses negatively affect agriculture by reducing animal productivity and health $(2,22)$. The ability of different ergot alkaloids to act as partial agonists or antagonists of various serotonin, dopamine, and $\alpha$-adrenaline receptors results in effects on nervous, circulatory, reproductive, and immune systems leading to high or low blood pressure, muscle contractions, reduced fertility, disturbances in sleep/wake cycles, lowered immune response, and, at high doses, hallucinations and gangrene of the extremities $(14,16,22,25,31)$.

Screens of other fungi for ergot alkaloids have identified several distantly related fungi as potential sources $(13,17,29)$. Among these fungi, the best characterized is Aspergillus fumigatus. This fungus was first noted to produce the ergot alkaloid festuclavine and two novel derivatives of festuclavine named fumigaclavine A and fumigaclavine B in semidefined broth culture (29). Later Cole et al. (3) described an additional festuclavine derivative, fumigaclavine $\mathrm{C}$, from broth cultures of $A$. fumigatus originally isolated from moldy silage.

Aspergillus fumigatus is associated with several human health issues. It is the most common airborne fungal pathogen of humans $(6,18)$. It can cause invasive aspergillosis in immunocompromised individuals, with resulting mortality at $>50 \%$. In 
immunocompetent individuals, the fungus can colonize preexisting cavities in the lungs or sinuses without penetrating into tissues, a condition known as aspergilloma or fungus ball $(6,11,18)$. The fungus also is associated with air quality issues in indoor environments and near composting facilities $(11,12,27)$. Presence of conidia of $A$. fumigatus and of several other fungi in such environments has been loosely associated with respiratory allergic symptoms and miscellaneous other ailments, but causal associations have not been demonstrated.

Most mycotoxin-producing fungi produce their toxins in the substrate in which they grow and ingestion of that contaminated substrate is required for intoxication. Although less frequently documented, the presence of mycotoxins in or on conidia of the producing fungus provides the potential for delivery of mycotoxins via the alternate and less voluntary route of inhalation. For example, the black mold fungus Stachybotrys chartarum contains trichothecenes associated with its spores (28).

Our objectives in this study were to determine if conidia of A. fumigatus contain ergot alkaloids in respirable form, to quantify ergot alkaloids associated with conidia produced on several environmentally relevant substrates, and to measure physical properties of conidia likely to affect their respirability. Detection and quantification of ergot alkaloids associated with conidia is prerequisite to investigations of potential contributions of the alkaloids to adverse human health effects or to other aspects of the biology of the fungus.

\section{MATERIALS AND METHODS}

Fungi and culture conditions. Aspergillus fumigatus isolate WVU1943 (FGSC) was cultured from the lungs of a deceased parakeet in Charleston, West Virginia, during 1977. 
Isolate WVU2026 (FGSC) was obtained by passive sampling of the air (by opening a Petri dish of potato dextrose agar for 20 minutes under ambient conditions) in a house in Monongalia County, West Virginia, during 2003. All viable cultures were manipulated in a class II biosafety cabinet. Isolates were maintained on potato dextrose agar [PDA; $20 \mathrm{~g} / \mathrm{L}$ dehydrated mashed potatoes (Idaho Spuds, Pillsbury, Minneapolis, MN), $20 \mathrm{~g} / \mathrm{L}$ glucose, $15 \mathrm{~g} / \mathrm{L}$ agar (Bacto-Agar, Difco, Detroit, MI)] at room temperature or at $37^{\circ} \mathrm{C}$, and most ergot alkaloid analyses were conducted on cultures grown on this medium. Additional alkaloid analyses were conducted with A. fumigatus isolates cultured on the following substrates: $1.5 \%(\mathrm{w} / \mathrm{v})$ water agar plates (prepared with Difco Bacto-Agar); Pittsburgh Paint (Pittsburgh, PA) interior latex base 88-110 (low luster semigloss latex $100 \%$ acrylic white and pastel mixing base) that had been applied with a brush in two thin layers on the inside of lids of Petri dishes and allowed to dry for $2 \mathrm{hr}$ prior to inoculation; maize (Zea mays DeKalb hybrid DK626RR, DeKalb, IL) seedlings four days after germination of surface-sterilized kernels on water agar; and, water-rinsed and autoclaved pistachio (Pistacia vera; Blue Diamond, Sacramento, CA) shells. Painted and inoculated Petri dish lids were placed over $1.5 \%$ water agar plates to promote high humidity for growth of the fungus. Pistachio shells were incubated in Petri dishes containing $8 \mathrm{~mL}$ of sterile distilled water to promote high humidity. Cultures were inoculated by lightly dusting the medium with conidia that had adhered to a Petri dish lid formerly covering a mature culture. In mock inoculations, an average of $1.2 \times 10^{7}$ conidia per Petri dish were deposited by this inoculation method.

Large numbers of conidia, free of culture medium and hyphae, were collected from PDA cultures by tapping a culture dish such that conidia accumulated on the inside 
of the lid. In this way, alkaloids could be extracted exclusively from conidia.

Alternatively, conidia could be made airborne from a Petri dish lid by agitating the lid by hand. Airborne conidia were then collected on a $0.2-\mu \mathrm{m}$ SFCA filter (Nalge Nunc International, Rochester, NY) or a $0.45-\mu \mathrm{m}$ nylon filter (Millipore, Bedford, MA) under vacuum and extracted by washing the filter with $800 \mu$ of $80 \%$ methanol. In other experiments, conidia were collected from water agar and paint cultures by very briefly washing small portions of the sporulating cultures with $800 \mu \mathrm{L}$ of $80 \%$ methanol. Conidia were harvested from corn or pistachio tissue by suspending pieces of the colonized and spore-bearing tissue in $1 \mathrm{~mL}$ of $80 \%$ methanol. Microscopic examination of conidial suspensions prepared from any of these alternate substrates revealed the lack of hyphae. However, with the exception of the conidia collected from PDA cultures, the extraction solvent had been briefly in contact with hyphae and the colonized substrate or medium.

Stachybotrys chartarum and Aspergillus nidulans were isolated from contaminated building materials; Aspergillus niger was isolated by passive air sampling (as described above for A. fumigatus). Conidia of these fungi, and of A. fumigatus, collected for measurement of their physical properties, were harvested with $80 \%$ methanol from cultures grown on PDA. The number of conidia per $\mathrm{mL}$ of suspension was determined by counting dilutions on a hemacytometer (Hausser Scientific, Horsham, PA). Conidia (of defined number) were collected by filtration though tared $0.22-\mu \mathrm{m}$ nylon filters (Costar, Corning, NY) and retained for mass determination on a MettlerToledo (Columbus, OH) AG104 balance after drying in a Labconco (Kansas City, MO) 
centrivap concentrator. Conidial diameter was determined microscopically with the aid of an ocular micrometer.

HPLC, chemical manipulations, and mass spectrometry. Aliquots of conidia were suspended in $80 \%$ methanol, transferred to $1.5-\mathrm{ml}$ microcentrifuge tubes, and rotated end-over-end at $40 \mathrm{rpm}$ for $1 \mathrm{hr}$ on a Glas-Col (Terre Haute, IN) mini-rotator. The number of conidia in the extraction tube was determined by counting dilutions on a hemacytometer. Conidia from PDA-based cultures were separated from the alkaloid extract by centrifugal filtration and weighed as described above. For conidia derived from cultures on alternate substrates, conidial mass was calculated as the product of the number of conidia counted in the sample and the value previously calculated for mass of an individual conidium. These calculations are based on the assumption that the mass of a conidium produced on these alternate substrates is the same as the mass of a conidium produced on PDA.

Methanol extracts of conidia (with no further preparation beyond removal of conidia by filtration) were analyzed by HPLC with conditions described previously (23) except for changes in the equipment used. The HPLC apparatus consisted of a model 600 pump controller with in-line degasser, model 717plus autosampler, model 2487 absorbance detector or model 996 photodiode array detector (all from Waters Corp., Milford, MA), and a Rainin (Woburn, MA) F12 fluorescence detector set with excitation and emission wavelengths of $272 \mathrm{~nm}$ and $372 \mathrm{~nm}$, respectively. Alkaloid quantities were determined based on comparison of peak areas relative to a standard curve derived from four dilutions each of three parallel samples of the highly similar ergot alkaloid 
agroclavine (Sigma, St. Louis, MO) and adjusted for relative molecular weights of analytes and standard.

For PDA-based cultures, several dilutions of each A. fumigatus spore extract were analyzed and the concentration of each alkaloid determined by linear regression analysis. Analyses of PDA-based cultures (Table 2) were conducted in triplicate on each of two separate occasions. Three additional preliminary analyses yielded similar results. Extracts from A. fumigatus-colonized plant materials, water agar, or paint-based cultures ( $n=3$ to $6 /$ medium / isolate), which were less dense and contained fewer conidia than PDA-based cultures, were analyzed without dilution.

Agroclavine was validated as a standard for the A. fumigatus clavines by comparing experimentally measured concentrations of it versus calculated concentrations of four separate preparations of dihydroergotamine and dihydroergocristine (Sigma), which carry the identical ergoline chromophore as the A. fumigatus ergot alkaloids. Concentrations of dihydroergotamine measured based on fluorescence relative to an agroclavine standard curve were $1.0 \pm 0.04($ mean $\pm \mathrm{SE})$ times the calculated values, whereas the measured concentrations of dihydroergocristine solutions were $0.98 \pm 0.05$ times the calculated values.

The relationships among compounds represented in different peaks were investigated by deacetylation of alkaloid fractions with $1 \mathrm{M} \mathrm{NaOH}$ at room temperature for $15 \mathrm{hr}(29)$. Base-treated samples were neutralized with an equinormal solution of acetic acid and analyzed by HPLC as described above. Masses of untreated and basetreated compounds were obtained on a Finnegan Mass Spectrometer (model SSQ7000) at the WVU Mass Spectrometry Center. Isolated peaks collected after HPLC were 
concentrated 10-fold under vacuum, introduced into the ion source with an infusion pump, and ionized in positive electrospray ionization mode.

Statistical Analyses. Data were analyzed by analysis of variance, and means were compared by the Tukey-Kramer honestly significant difference test in the JMP software package (SAS Institute, Cary, NC). Sets of values having unequal variances were log-transformed prior to analysis.

\section{RESULTS}

\section{Identification and quantification of ergot alkaloids associated with conidia of $A$.}

fumigatus. Several compounds from simple $80 \%$ methanolic extracts of conidia from PDA-cultured A. fumigatus produced large fluorescent peaks when analyzed by HPLC with excitation and emission wavelengths of $272 \mathrm{~nm}$ and $372 \mathrm{~nm}$, respectively (Fig. 1). Electrospray ionization-mass spectrometry (ESI-MS) of the compound in peak C (Fig. 1), which had the greatest area and was the most nonpolar of the alkaloids analyzed, revealed an ion at $m / z 367$ corresponding in mass to [fumigaclavine $\mathrm{C}+\mathrm{H}]^{+}$, which had previously been described from broth cultures of A. fumigatus (3). Treatment of this compound with $1 \mathrm{M} \mathrm{NaOH}$ resulted in a more polar compound (peak dC, Fig. 1) that yielded an ion at $\mathrm{m} / \mathrm{z} 325$, corresponding in mass to [deacetylated fumigaclavine $\mathrm{C}+\mathrm{H}]^{+}$.

Similarly the compound represented by peak A (Fig. 1) yielded an ion of $\mathrm{m} / \mathrm{z} 299$ in its native form, consistent with a proposed identity as fumigaclavine A, and was converted after treatment with $\mathrm{NaOH}$ to a more polar compound (peak dA, Fig. 1) with an ion of $m / z 257$, consistent with [deacetylated fumigaclavine $\mathrm{A}+\mathrm{H}]^{+}$. Peak B, which cochromatographed with experimentally deacetylated fumigaclavine A (Fig. 1), is proposed to be fumigaclavine $\mathrm{B}$, which is identical to deacetylated fumigaclavine $\mathrm{A}$ (3, 
29). Small quantities of fumigaclavine $\mathrm{B}$ also were detectable in untreated extracts of $A$. fumigatus conidia (peak B, Fig. 1). The compound contained within peak F (Fig. 1) yielded an ion at $m / z 241$ in ESI-MS and was unaffected by $\mathrm{NaOH}$ treatment. These characteristics are consistent with it being festuclavine, which had been characterized previously from culture extracts of A. fumigatus (29).

Absorbance spectra of each of these compounds (data not shown) and their relative retention times on $\mathrm{C} 18$ silica were consistent with their proposed identities. Analyses of these same conidial extracts with an excitation wavelength of $310 \mathrm{~nm}$ and emission wavelength of $410 \mathrm{~nm}$ did not detect any of the previously characterized lysergyl-derived ergot alkaloids (not shown). Conidia collected on the inner lid of a Petri dish and conidia aerosolized and collected on filters under vacuum yielded the same spectrum of ergot alkaloids.

To relate quantities of ergot alkaloids to spore mass and investigate the buoyancy of A. fumigatus conidia, physical properties of conidia were measured (Table 1). The conidia of A. fumigatus were significantly smaller, lighter, and less dense than those of some other common Aspergillus species and the black mold fungus $S$. chartarum (Table 1). [Although the ranges of diameter for conidia of Aspergillus species have been well established (26), we measured means and report them here so that calculations of density would be made from volume and mass of the same collections of conidia.] Cultures of $A$. fumigatus on PDA sporulate abundantly; a two-month-old, 85 -mm culture contained 5.8 $\times 10^{10}$ conidia.

Each of the four defined ergot alkaloids was quantified in extracts of defined conidium number and conidium mass (Table 2). Ergot alkaloids accounted for $1.1 \%$ of 
the mass per conidium of $A$. fumigatus isolate WVU1943 grown on PDA, whereas conidia of isolate WVU2026 appeared to contain slightly lower quantities of ergot alkaloids, though the difference was not statistically significant. Both isolates accumulated the same array of ergot alkaloids when cultured on latex-based paint (Table 2). Isolate WVU2026 contained significantly more total ergot alkaloids on paint than it did on PDA; isolate WVU1943 also contained abundant total ergot alkaloid on paint but the increase relative to PDA was not statistically significant. Colonies on paint were relatively small and consisted mainly of conidiophores and conidia with very little vegetative hyphae. Cultures on water agar (also mostly conidiophores and conidia) still accumulated ergot alkaloids despite the limited exogenous nutrients in this medium. However, concentrations of ergot alkaloids extracted from the water agar-derived conidia were generally lower than those from conidia produced on the other substrates. The same set of ergot alkaloids also was detected in or on conidia of cultures grown on cultured maize seedlings (on which the fungus colonized mainly the residual scutellum and kernel tissue), where they accumulated to relatively high levels, and on sterilized pistachio shells (on which the fungus grew poorly), where the concentrations of ergot alkaloids were relatively low (Table 2 ).

The yield of ergot alkaloids recovered after a more harsh extraction procedure was assessed in a separate experiment. Breaking of $\sim 63 \%$ of the conidia by bead-beating, followed by a 1 -hr extraction in $80 \%$ methanol resulted in a $38 \%$ increase in total ergot alkaloids relative to those extracted from unbeaten conidia. 


\section{DISCUSSION}

Our results demonstrate that high levels of certain ergot alkaloids are associated with conidia of A. fumigatus. The alkaloids are present in or on conidia produced by cultures grown on a variety of substrates. Moreover, the conidia of A. fumigatus are smaller, lighter, and less dense than those of closely related species. These physical properties may promote the aerosolization and buoyancy of the conidia, which serve as vehicles for the alkaloids.

Whether the ergot alkaloids are on the surface of the conidia, contained within the conidia, or in both locations cannot be answered definitively with the available data. The majority of the ergot alkaloids were easily extracted from intact conidia, which suggests a surface location. A bead-beating treatment that physically disrupted the spore wall increased the amount of alkaloid extracted by $38 \%$. However, in addition to cell breakage, this treatment also more vigorously extracts any surface compounds. The data from this more disruptive extraction also indicate that the values listed in Table 2 represent minimum values for conidium-associated alkaloids. The question of location of the ergot alkaloids relative to the spore surface may not be significant from a health perspective. In immunocompetent individuals, inhaled conidia are likely to be killed and lysed by macrophages (18), releasing any mycotoxins contained within. Conidia that are not killed and lysed present a threat of infection and, likely, additional toxin production. A role for ergot alkaloids in invasive aspergillosis has not been investigated. Such studies would be facilitated by comparison of wild-type isolates with the ergot alkaloiddeficient mutants described in the accompanying paper (4). In the absence of infection, conidia may serve as vehicles for exposure to ergot alkaloids. 
The issue of health risks posed by inhalation of mycotoxin-containing conidia is complex and affected by several factors including the physical nature of the conidia with respect to their potential for dispersal and inhalation and the production of mycotoxins on environmentally relevant substrates. The conidia of A. fumigatus have properties that appear to facilitate their dispersal and inhalation. The $2.8-\mu \mathrm{m}$ diameter of conidia is small enough for them to penetrate deep into the alveoli of the lungs (18). The low specific gravity of the conidia (0.24) probably promotes efficient air dispersal. For comparison, the conidia of the trichothecene producer $S$. chartarum, which have generated considerable health concerns $(10,28)$, have a mass that is 48 times greater and a specific gravity that is 4 times greater than those of conidia of A. fumigatus.

Ergot alkaloids were detected on all tested media, including environmentally relevant substrates such as latex paint, two different plant materials, and water agar (representative of moist, nutritionally poor environments). The reported concentrations of ergot alkaloids associated with conidia produced on substrates other than PDA are based on the number of conidia extracted and a value for conidial mass measured from conidia produced on PDA. Thus, these values are based on the assumption that conidia produced on any of the alternate substrates have the same mass as conidia produced on PDA. We contend that this is a reasonable assumption. However, if the mass per conidium were, in fact, greater on a natural substrate than on PDA, then the calculated values of ergot alkaloid per conidium would be lower. Conversely, if the conidial mass were lower on a natural substrate than on PDA, then alkaloid concentration would be greater than listed in Table 2. 
A more complicated factor in determination of health risks associated with conidial mycotoxins is the issue of whether the toxins can or will be encountered in quantities sufficient for them to exert their effects. There are at least three components to this issue, including (a) concentration of mycotoxin per conidium, (b) the number of conidia encountered, and (c) the toxicity of the mycotoxins.

Concentrations of ergot alkaloids exceeding $1 \%$ by mass of $A$. fumigatus conidia are relatively high for fungal natural products. There are few data available for direct comparison, because most mycotoxin analyses are expressed as mass of toxin per unit volume of culture. Data on respirable trichothecenes from $S$. chartarum (28) indicate a mean of $17 \mathrm{ng}$ of trichothecenes per mg of dust aerosolized from $S$. chartarum cultured on rice. Assuming that conidia, which constituted $85 \%$ of the particles in the aerosolized dust (28), contribute $85 \%$ of the mass of the dust sample, then $0.002 \%$ of the conidium by mass was trichothecenes. With a different isolate of this same fungal species and measuring only the most abundant trichothecene, Nikulin et al. (21) reported $0.1 \mathrm{pg}$ of satratoxin $\mathrm{H}$ per conidium, which would translate to $0.0007 \%$ satratoxin $\mathrm{H}$ per mass of conidium. Another source of alkaloid data per unit fungal mass comes from the poisonous mushroom literature. For example, basidiocarps of Amanita muscaria contain ibotenic acid at $0.45 \%$ by mass dry weight and muscimol at $0.036 \%(30)$ [assuming 12.5 mg fresh weight basidiocarp yields $1 \mathrm{mg}$ dry weight (9)]. Amatoxins have been measured at $0.1 \%$ to $0.7 \%$ of the dry weight of Amanita phalloides basidiocarps (9). In a more relevant example, ergot sclerotia of field-grown $C$. purpurea contain $1 \%$ to $2 \%$ ergot alkaloids by mass $(5,20)$. 
The number of $A$. fumigatus conidia available in the air depends on the substrate and the environment in which the fungus is growing. Under favorable conditions the fungus can sporulate prolifically. A typical culture of A. fumigatus on PDA can yield $\sim 10^{9}$ conidia per $\mathrm{cm}^{2}$ of culture surface area. Viable A. fumigatus conidia per $\mathrm{m}^{3}$ of air range from 0 to $200 \mathrm{CFU}$ in clean environments (27), to $10^{7} \mathrm{CFU}$ near composting facilities (12), to $10^{11} \mathrm{CFU}$ near moldy hay or other stored organic materials (27). Considering an intake rate of $0.63 \mathrm{~m}^{3}$ of air per $\mathrm{hr}(32)$, a conidial ergot alkaloid content of $1 \%$ by mass, and no further ergot alkaloid production after inhalation of the fungus, ergot alkaloid dose would range from $3.7 \mathrm{pg}$ per $\mathrm{hr}$ (at $200 \mathrm{CFU} / \mathrm{m}^{3}$ ), to $180 \mathrm{ng}$ per $\mathrm{hr}$ (at $10^{7} \mathrm{CFU}$ per $\mathrm{m}^{3}$ ), to $1.8 \mathrm{mg}$ per $\mathrm{hr}\left(\right.$ at $10^{11} \mathrm{CFU}$ per $\left.\mathrm{m}^{3}\right)$. An interesting point of reference is that an ingested dose of the illicit and highly active ergot alkaloid LSD can range from hundreds of ng to hundreds of $\mu \mathrm{g}(1)$, but based on US Drug Enforcement Agency data is frequently in the 20 to $80 \mu \mathrm{g}$ range (http://www.nida.nih.gov/Infofax/lsd.html). To inhale a comparable mass of $A$. fumigatus ergot alkaloids in one hour would require exposure to $10^{7}$ to $10^{10} \mathrm{CFU}$ per $\mathrm{m}^{3}$. Such high concentrations of conidia will be encountered only rarely. A more practical issue to consider is whether there are potential health effects of a less remarkable nature (e.g., effects on depression, blood pressure, or sleep/wake cycles) associated with chronic daily doses of ergot alkaloids in the ng to $\mu \mathrm{g}$ range. This issue has not been investigated.

Toxicity of the particular ergot alkaloids associated with the A. fumigatus conidia has not been studied extensively, but the available data suggest that these mycotoxins have considerable biological activity. Similar to other studied ergot alkaloids, festuclavine interacts with receptors for serotonin, dopamine, and $\alpha$-adrenaline $(7,14,24$, 
25). Festuclavine and synthetic derivatives of festuclavine also are cytostatic in in vitro assays with several bacteria and mouse lymphoma cell lines $(7,8)$. Moreover, festuclavine is unique among the naturally occurring ergot alkaloids in that it is directly mutagenic in the Ames test (15). In the sole published animal study of fumigaclavine $\mathrm{C}$, ingestion of relatively crude preparations of this ergot alkaloid greatly reduced feed intake of treated calves, and caused hemorrhagic enteritis of small and large intestines as well as patchy interstitial thickening of alveolar walls of the animals (3).

Demonstration of the presence of ergot alkaloids at relatively high concentrations in or on conidia of A. fumigatus does not ipso facto indicate a role for the toxins in pathogenesis, other health effects, or any specific aspect of the biology of the fungus. However, it does raise interesting questions for further research. Studies with ergot alkaloid-deficient mutants such as the $d m a W$-knockout strain described in the accompanying paper (4) should be useful for assessing the contribution of ergot alkaloids to virulence towards animals or potential contributions of the alkaloids to the ecological success of A. fumigatus. Since minimization of the mass of a conidium appears to have been selected for in this fungus, the inclusion of alkaloids in quantities that exceed $1 \%$ of the mass of the conidium should have been selected against unless they provided some advantage to the fungus.

\section{ACKNOWLEDGEMENTS}

This work was supported by USDA-NRI grant No. 2001-3531 9-10930 and Hatch funds, and was published with the approval of the Director of the WV Agricultural and Forestry Experiment Station as Scientific Article no. 2895. We thank Robert Smith of the WVU Mass Spectrometry Center for assistance with mass spectrometry, Barton Baker and Jim 
Kotcon for helpful discussions, and Brian Lewis and Jessica Cipoletti for technical assistance. 


\section{REFERENCES}

1. Baudot, P., and J. C. Andre. 1985. Identification and quantitative determination of LSD by fluorescence: new data. Bull. Narc. 37:79-94.

2. Clay, K., and C. Schardl. 2002. Evolutionary origins and ecological consequences of endophyte symbiosis with grasses. Am. Nat. 160:S199-S127.

3. Cole, R. J., J. W. Kirksey, J. W. Dorner, D. M. Wilson, J. C. Johnson Jr., A. N. Johnson, D. M. Bedell, J. P. Springer, K. K. Chexal, J. C. Clardy, and R. H. Cox. 1977. Mycotoxins produced by Aspergillus fumigatus species isolated from molded silage. J. Agric. Food Chem. 25:826-830.

4. Coyle, C. M., and D. G. Panaccione. 2005. An ergot alkaloid biosynthesis gene and clustered hypothetical genes from Aspergillus fumigatus. Appl. Environ. Microbiol. 71:3112-3118.

5. Cvak, L. 1999. Industrial production of ergot alkaloids, p. 373-409. In V. Kren, and L. Cvak (ed.), Ergot: The Genus Claviceps. Harwood Academic Publishers, The Netherlands.

6. Denning, D. W. 1998. Invasive aspergillosis. Clin. Infect. Dis. 26:781-805.

7. Eich, E., and H. Pertz. 1999. Antimicrobial and antitumor effects of ergot alkaloids and their derivatives, p. 411-440. In V. Kren, and L. Cvak (ed.), Ergot: The Genus Claviceps. Harwood Academic Publishers, The Netherlands.

8. Eich, E., C. Becker, and W. E. G. Müller. 1984. Clavines - new antibiotics with cytostatic activity. Biochem. Pharmacol. 33:523-526. 
9. Enjalbert, F., C. Gallion, F. Jehl, H. Monteil, and H. Faulstich. 1993. Amatoxins and phallotoxins in Amanita species: high-performance liquid chromatography determination. Mycologia 85:579-584.

10. Etzel, R. A., E. Montana, W. G. Sorenson, G. J. Kullman, T. M. Allan, and D. G. Dearborn. 1998. Acute pulmonary hemorrhage in infants associated with exposure to Stachybotrys atra and other fungi. Arch. Pediatr. Adolesc. Med. 152:757-762.

11. Fischer, G., and W. Dott. 2003. Relevance of airborne fungi and their secondary metabolites for environmental, occupational and indoor hygiene. Arch. Microbiol. 179:75-82.

12. Fischer, G., T. Muller, R. Schwalbe, R. Ostrawski, and W. Dott. 2000. Speciesspecific profiles of mycotoxins produced in cultures and associated with conidia of airborne fungi derived from biowaste. Int. J. Hyg. Environ. Health 203:105-116.

13. Flieger, M., M. Wurst, and R. Shelby. 1997. Ergot alkaloids - sources, structures, and analytical methods. Folia Microbiol. 42:3-30.

14. Fuxe, K., B. B. Fredholm, L. F. Agnati, S.-O. Ögren, B. J. Everitt, G. Jonsson, and J.-̈̈. Gustafsson. 1978. Interactions of ergot drugs with central monoamine systems. Pharmacology 16:99-134.

15. Glatt, H., H. Pertz, R. Kasper, and E. Eich. 1992. Clavine alkaloids and derivatives as mutagens detected in the AMES test. Anti-Cancer Drugs 3:609-614.

16. Gröger, D., and H. G. Floss. 1998. Biochemistry of ergot alkaloids - achievements and challenges. Alkaloids 50:171-218. 
17. Kozlovsky, A. G. 1999. Producers of ergot alkaloids out of Claviceps genus, p. 479-499. In V. Kren, and L. Cvak (ed.), Ergot: The Genus Claviceps. Harwood Academic Publishers, The Netherlands.

18. Latgé, J.-P. 1999. Aspergillus fumigatus and aspergillosis. Clin. Microbiol. Rev. 12:310-350.

19. Matossian, M. K. 1989. Poisons of the Past: Molds, Epidemics, and History. Yale University Press, New Haven, CT. 190 p.

20. Németh, E. 1999. Parasitic production of ergot alkaloids, p. 303-319. In V. Kren, and L. Cvak (ed.), Ergot: The Genus Claviceps. Harwood Academic Publishers, The Netherlands.

21. Nikulin, M., K. Reijula, B. B. Jarvis, and E. L. Hintikka. 1996. Experimental lung mycotoxicosis in mice induced by Stachybotrys atra. Intl. J. Exp. Path. 77:213218.

22. Panaccione, D. G., and C. L. Schardl. 2003. Molecular genetics of ergot alkaloid biosynthesis, p. 399-424. In J. F. White Jr., C. W. Bacon, N. L. Hywel-Jones, and J. W. Spatafora (ed.), The Clavicipitalean Fungi: Evolutionary Biology, Chemistry, Biocontrol, and Cultural Impacts. Marcel-Dekker, New York.

23. Panaccione, D. G., B. A. Tapper, G. A. Lane, E. Davies, and K. Fraser. 2003. Biochemical outcome of blocking the ergot alkaloid pathway of a grass endophyte. J. Agric. Food Chem. 51:6429-6437.

24. Pertz, H. 1996. Naturally occurring clavines: antagonism/partial agonism at 5-HT $2 \mathrm{~A}$ receptors and antagonism at $\alpha 1$-adrenoceptors in blood vessels. Planta Med. 62:387392. 
25. Pertz, H., and E. Eich. 1999. Ergot alkaloids and their derivatives as ligands for serotoninergic, dopaminergic, and adrenergic receptors, p. 411-440. In V. Kren, and L. Cvak (ed.), Ergot: The Genus Claviceps. Harwood Academic Publishers, The Netherlands.

26. Raper, K. B., and D. I. Fennell. 1965. The Genus Aspergillus. Williams \& Wilkins, Balitmore, MD. 686 p.

27. Solomon, W. R., and H. A. Burge. 1984. Allergens and pathogens in indoor air quality, p. 173-191. In P. J. Walsh, C. S. Dudney, and E. D. Copenhaver (ed.), Indoor Air Quality. CRC Press, Boca Raton, FL.

28. Sorenson, W. G., D. G. Fraser, B. B. Jarvis, J. Simpson, and V. A. Robinson. 1987. Trichothecene mycotoxins in aerosolized conidia of Stachybotrys atra. Appl. Environ. Microbiol. 53:1370-1375.

29. Spilsbury, J. F., and S. Wilkinson. 1961. The isolation of festuclavine and two new clavine alkaloids from Aspergillus fumigatus Fres. J. Chem. Soc. 5:2085-2091.

30. Tsunoda, K., N. Inoue, Y. Aoyagi, and T. Sugahara. 1993. Changes in concentration of ibotenic acid and muscimol in the fruit body of Amanita muscaria during the reproduction stage. J. Food Hyg. Soc. Japan 34:18-24

31. Tudzynski, P., T. Correia, and U. Keller. 2001. Biotechnology and genetics of ergot alkaloids. Appl. Microbiol. Biotechnol. 57:593-605.

32. US Environmental Protection Agency. 1997. Volume I: General Factors. Exposure Factors Handbook. EPA Office of Research and Development Publication EPA/600/P-95/002Fa-c. Washington, DC. 


\section{FIGURE LEGEND}

Figure 1: Chromatography of ergot alkaloids in untreated $80 \%$ methanolic extract and $\mathrm{NaOH}$-treated methanolic extract of conidia of A. fumigatus WVU1943 cultured on PDA. Detection was by fluorescence with excitation and emission wavelengths of $272 \mathrm{~nm}$ and $372 \mathrm{~nm}$, respectively. $\mathrm{B}=$ fumigaclavine $\mathrm{B} ; \mathrm{F}=$ festuclavine; $\mathrm{A}=$ fumigaclavine $\mathrm{A} ; \mathrm{C}=$ fumigaclavine $\mathrm{C} ; \mathrm{dA}=$ deactylfumigaclavine $\mathrm{A} ; \mathrm{dC}=$ deactylfumigaclavine $\mathrm{C}$. 


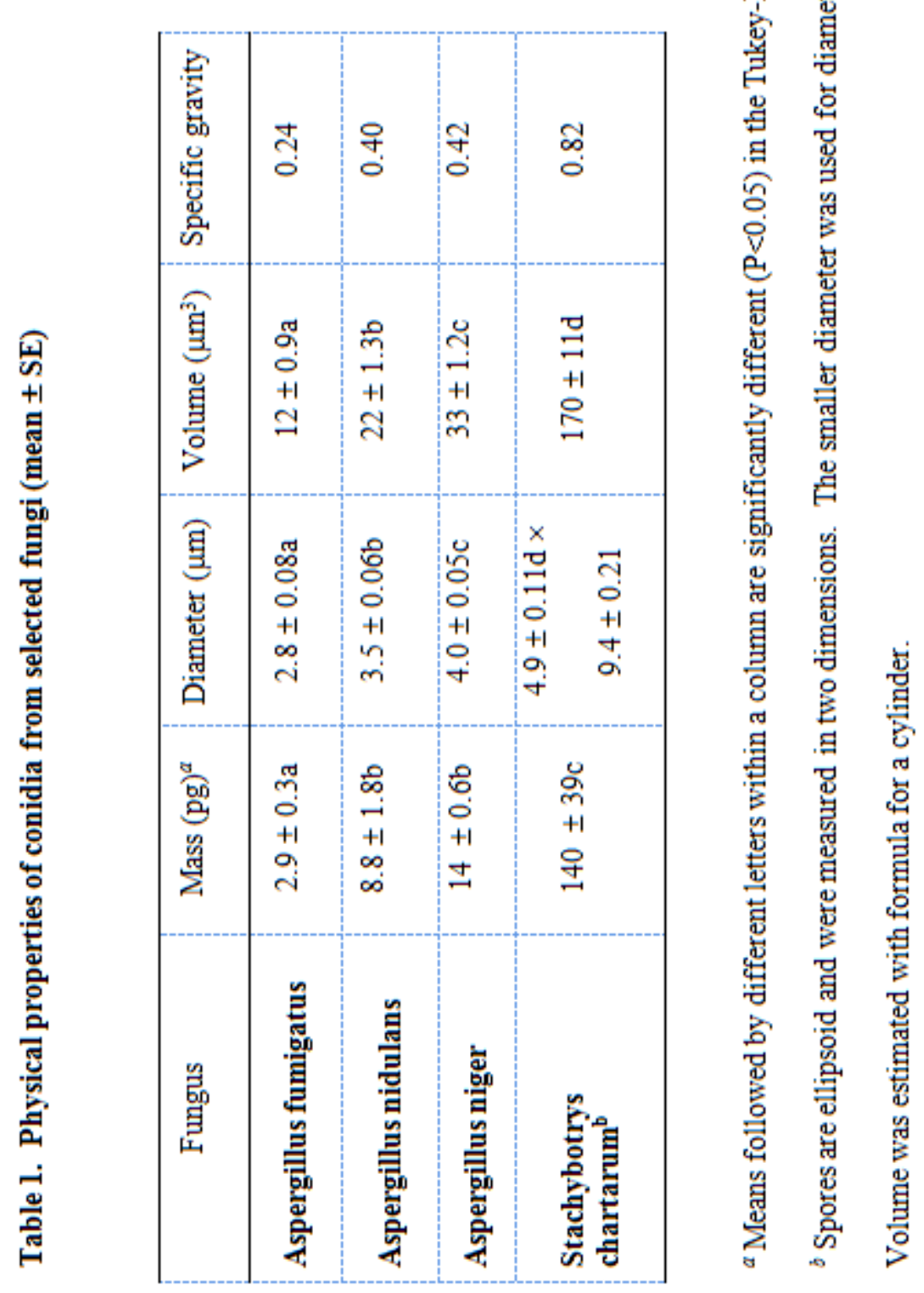

Appendix 1 - Table 1 


\begin{tabular}{|c|c|c|c|c|c|c|c|c|c|}
\hline 氶 & $\begin{array}{l}\text { a्ल } \\
\text { mे } \\
\text { m } \\
+1 \\
= \\
=\end{array}$ & $\begin{array}{l}0 \\
\stackrel{0}{n} \\
+1 \\
r \\
i\end{array}$ & 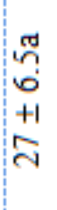 & $\begin{array}{l}\text { o } \\
\sim \\
+1 \\
+1 \\
-1\end{array}$ & \begin{tabular}{l}
0 \\
\multirow{2}{0}{} \\
0 \\
+1 \\
m \\
$\stackrel{c}{c}$
\end{tabular} & 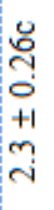 & $\begin{array}{l}\text { 足 } \\
\stackrel{+1}{ \pm} \\
\pm\end{array}$ & 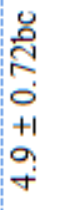 & \\
\hline 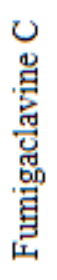 & $\begin{array}{l}\text { त्ञ } \\
\text { ले } \\
\text { +1 } \\
\text { a } \\
\text { a }\end{array}$ & $\begin{array}{l}\frac{\partial}{0} \\
\stackrel{0}{1} \\
+1 \\
\infty \\
\text { m }\end{array}$ & $\begin{array}{l}\text { ra } \\
\text { r } \\
+1 \\
0 \\
0\end{array}$ & $\begin{array}{l}\text { त् } \\
\stackrel{+}{+} \\
+1 \\
\stackrel{\infty}{\infty}\end{array}$ & $\begin{array}{l}\text { D } \\
\text { m. } \\
\text { +1 } \\
\text { cy } \\
\text {-1 }\end{array}$ & 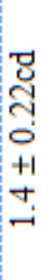 & 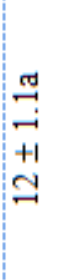 & $\begin{array}{l}0 \\
\text { N } \\
n \\
0 \\
+1 \\
0 \\
0 \\
+\end{array}$ & \\
\hline 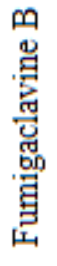 & 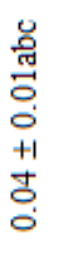 & $\begin{array}{l}0 \\
0 \\
0 \\
+1 \\
0 \\
0 \\
0\end{array}$ & $\begin{array}{c}0 \\
0 \\
0 \\
+1 \\
m \\
0 \\
0\end{array}$ & $\begin{array}{l}0 \\
0 \\
0 \\
0 \\
+1 \\
\infty \\
0 \\
0\end{array}$ & $\begin{array}{l}0 \\
8 \\
0 \\
0 \\
+1 \\
0 \\
0 \\
0\end{array}$ & $\begin{array}{l}0 \\
8 \\
0 \\
+1 \\
+1 \\
0 \\
0\end{array}$ & $\begin{array}{l}0 \\
0 \\
0 \\
0 \\
0 \\
+1 \\
0 \\
0 \\
0\end{array}$ & $\begin{array}{l}0 \\
0 \\
0 \\
+1 \\
8 \\
0 \\
0\end{array}$ & \\
\hline 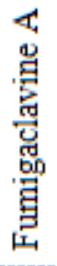 & $\begin{array}{l}\text { 영 } \\
\text { o } \\
+1 \\
\text { ㅇ } \\
\text { ले }\end{array}$ & $\begin{array}{l}8 \\
0 \\
0 \\
+1 \\
\text { N } \\
0 \\
0\end{array}$ & $\begin{array}{l}0 \\
0 \\
0 \\
+1 \\
+1 \\
\stackrel{2}{n}\end{array}$ & $\begin{array}{c}\text { त̃ } \\
0 \\
0 \\
+1 \\
0 \\
-1\end{array}$ & $\begin{array}{l}\text { fo } \\
0 \\
0 \\
+1 \\
\text { - } \\
0\end{array}$ & $\begin{array}{l}\text { Oे } \\
0 \\
+1 \\
0 \\
0 \\
0\end{array}$ & 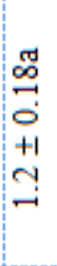 & 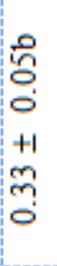 & 范 \\
\hline $\begin{array}{l}\text { 岂 } \\
\text { 营 } \\
\text { 苞 } \\
\text { 总 }\end{array}$ & $\begin{array}{l}\overrightarrow{0} \\
0 \\
0 \\
0 \\
+1 \\
+1 \\
0 \\
0\end{array}$ & $\begin{array}{l}0 \\
0 \\
0 \\
0 \\
+1 \\
n \\
-1\end{array}$ & $\begin{array}{l}\text { त̃ } \\
\text { +1 } \\
+1 \\
\sim\end{array}$ & 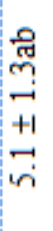 & 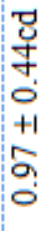 & $\begin{array}{l}0 \\
0 \\
0 \\
0 \\
+1 \\
-1 \\
0\end{array}$ & $\begin{array}{c}0 \\
0 \\
0 \\
+1 \\
\text { ले } \\
0 \\
0\end{array}$ & 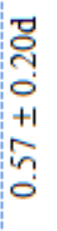 & 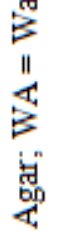 \\
\hline $\begin{array}{l}\text { 罳 } \\
\text { 爱 }\end{array}$ & 皃 & 㟔 & 菬 & 范 & $\stackrel{4}{\beta}$ & $\stackrel{4}{3}$ & $\begin{array}{l}\text { N } \\
\text { 㟧 }\end{array}$ & $\begin{array}{l}\text { 恶 } \\
\text { 岂 } \\
\text { 己. }\end{array}$ & 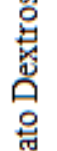 \\
\hline $\begin{array}{l}\text { 节 } \\
\text { 总 }\end{array}$ & 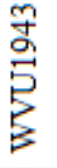 & 怘 & $\frac{9}{3}$ & క్రి & 等 & 兽 & 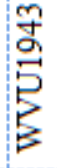 & $\frac{9}{5}$ & 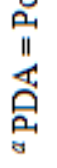 \\
\hline
\end{tabular}

Appendix 1 - Table 2 


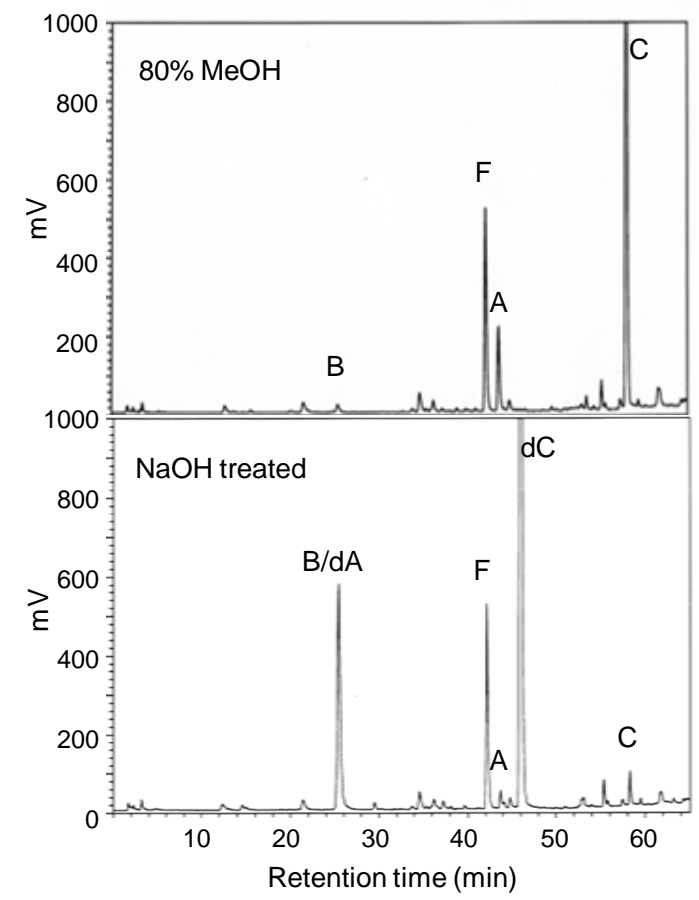

Panaccione \& Coyle, Fig. 1

Appendix 1 - Figure 1 


\section{APPENDIX 2: Analysis of ergot alkaloids in spore only samples versus entire fungal (plate) cultures in the fungus, Aspergillus fumigatus}

\section{INTRODUCTION}

Cultures of Aspergillus fumigatus consist of spores, conidiophores (asexual sporulation structures containing a stalked vesicle and a layer of phialides), and mycelia (vegetative growth). Ergot alkaloids accumulate in high concentrations in or on spores of $A$. fumigatus, and production of ergot alkaloids is restricted to sporulating cultures of the fungus $[(1,3)$; Ch. 4, appendix 1]. Preliminary work indicated that extracts from spores contained a different ratio of the four major A. fumigatus ergot alkaloids than extracts made from plate cultures, containing all four cell types. This observation raises the possibility that ergot alkaloids may be produced in cell types other than spores or in addition to spores. The location of the production of alkaloids and the possibility of accumulation in other cell types has not yet been determined. The purpose of this experiment was to compare ergot alkaloid accumulation in spore only extracts to extracts from plate cultures to gain insight into whether these alkaloids are present in other cell types of sporulating cultures.

\section{MATERIALS AND METHODS}

Ten plates of A. fumigatus isolate WVU1943 were cultured on potato dextrose agar (PDA) for two weeks at $37^{\circ} \mathrm{C}$ as previously described (3). All manipulations were conducted in a Class II safety cabinet. Agar plugs containing $50.3 \mathrm{~mm}^{2}$ of colony surface area from each of the ten A. fumigatus plate cultures were extracted in $1 \mathrm{~mL}$ of $80 \%$ methanol as described by Coyle et al. (1). To analyze spore only samples, plates were inverted, gently agitated and the abscised conidia that had collected on the inner 
surface of the lid of each plate $(\mathrm{n}=10)$ were suspended in $1 \mathrm{~mL}$ of $80 \%$ methanol and extracted as above. Aliquots were removed for ergot alkaloid analysis and enumeration of conidia for each sample.

Ergot alkaloids present in each sample were identified and quantified by reversephase HPLC analysis with fluorescent detection. Peak identities were established based on work done by Panaccione and Coyle (3) together with elimination of those peaks by disruption of $d m a W$, the determinant step in the ergot alkaloid pathway [(2); Chapter 1]. Quantification of ergot alkaloids was based on comparison of peak areas relative to an external standard as previously described (3). Conidia present in dilutions of the initial methanol extracts were counted on a hemacytometer and visualized by light microscopy. Proportions of each individual ergot alkaloid were determined relative to total alkaloids in the sample. Comparison of conidia-only extracts versus entire culture extracts was done by application of the Wilcoxon / Kruskal-Wallis (rank sum) test present in the JMP software package (SAS, Cary, NC). This nonparametric test was chosen due to the unequal variance within some data sets.

\section{RESULTS}

Samples from the entire culture (and thus containing all four cell types) produced on average $3.26 \mathrm{fg}$ of ergot alkaloids per conidium, whereas samples made up exclusively of mature, abscised conidia contained an average of $1.21 \mathrm{fg}$ of ergot alkaloids per conidium. The difference in accumulation of total ergot alkaloids per spore is statistically significant $(\mathrm{p}=0.002)$

The proportion of festuclavine relative to total alkaloids extracted was significantly greater in plate extracts versus mature spore extracts $(p=0.0002)$ (Fig.1). 
The converse was true for the other three alkaloids, fumigaclavines B ( $p=0.0002), A$ $(\mathrm{p}=0.0025)$ and $\mathrm{C}(\mathrm{p}=0.0002)($ Fig. 1$)$

\section{DISCUSSION}

It has been shown previously that ergot alkaloids accumulate in high concentrations in or on spores of A. fumigatus (3) and that production of ergot alkaloids is restricted to sporulating cultures (1). The increase in total clavines per spore within samples comprised of all cell types versus only mature spores suggests that ergot alkaloids are also associated with other cell types within conidiating cultures. The increase in percentage of festuclavine and the decrease in proportion of downstream fumigaclavines within these multicell-type culture extracts relative to mature spore extracts suggest that festuclavine may be produced prior to spore formation and that the final stages in fumigaclavine production occur preferentially in spores. Whether there is discrete compartmentalization of these activities or simply an enrichment of enzymes for particular steps in different cell types has not been determined. 


\section{REFERENCES}

1. Coyle, C. M., S. C. Kenaley, W. R. Rittenour, and D. G. Panaccione. 2007. Association of ergot alkaloids with conidiation in Aspergillus fumigatus. Mycologia 99:804-811.

2. Coyle, C. M., and D. G. Panaccione. 2005. An ergot alkaloid biosynthesis gene and clustered hypothetical genes from Aspergillus fumigatus. Appl. Environ. Microbiol. 71:3112-3118.

3. Panaccione, D. G., and C. M. Coyle. 2005. Abundant respirable ergot alkaloids from the common airborne fungus Aspergillus fumigatus. Appl. Environ. Microbiol. 71:3106-3111. 


\section{FIGURE LEGEND}

Figure 1: Proportion of each individual ergot alkaloid relative to total alkaloids

extracted. A significant difference was found for each alkaloid using the nonparametric Wilcoxon / Kruskal-Wallis rank sum test. Error bars represent standard error. The insert represents a magnified view of proportions of fumigaclavine A and B. 


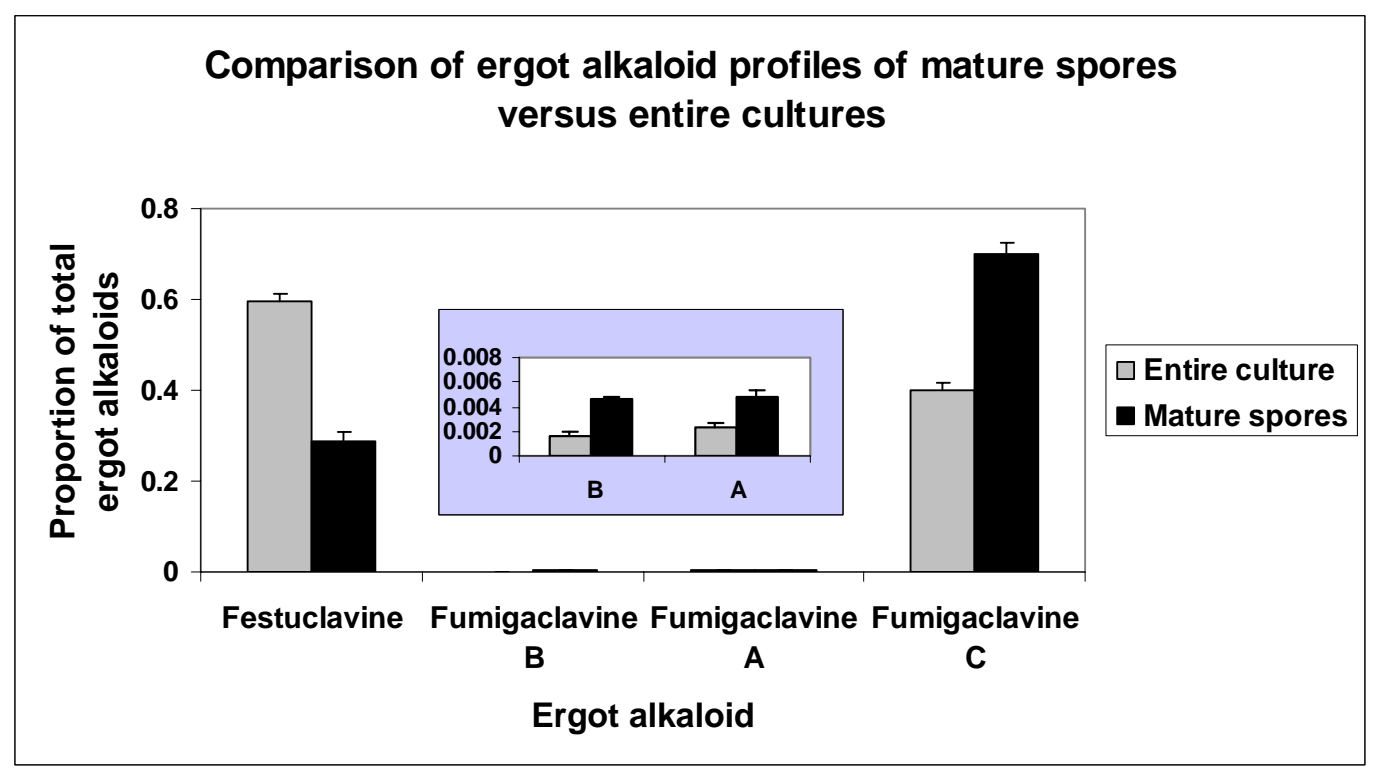

Appendix 2 - Figure 1 


\section{CURRICULUM VITAE}

\section{CHRISTINE M. COYLE}

50 Fitzwilliams Road, Washington, PA 15301 | 724-229-5089 | ccoyle11@gmail.com

\section{EDUCATION}

West Virginia University, Morgantown, WV

Ph.D. in Genetics and Developmental Biology - Spring 2009 [Expected]

Dissertation: "Characterization of the Ergot Alkaloid Biosynthesis Pathway in the Pathogenic Fungus Aspergillus fumigatus"

University of Maryland Baltimore County, Baltimore, MD

B.S. in Biochemistry and Molecular Biology - 2003

Honors: Cum Laude

\section{TEACHING EXPERIENCE}

West Virginia University, Morgantown, WV

Guest Lectures - “General Microbiology" [AEM 341] -2007, 2008

Topic: Microbial Genetics and Microbial Metabolism

Guest Lecture - “The Human Environment" [BIOL 115] - 2007

Topic: Genetically Modified Foods

Teaching Assistant - “Physiology of Fungi” [PPTH 730] - 2007

Prepared lab, instructed laboratory sessions, and met with students upon request

Teaching Assistant - "Principles of Biology” [BIOL 115] - 2003

Lectured/instructed laboratory sessions, met with students upon request, and graded all written work

Genetics Seminar - Prepared and presented 7 seminars - 2004-2008

Journal Club - Discussion of refereed publications regarding current concepts in environmental microbiology - 8 presentations - 2004-2008

\section{RESEARCH EXPERIENCE}

West Virginia University, Morgantown, WV

Research Assistant - 2004 - present

Chemical analysis by high performance liquid chromatography (HPLC), multiple molecular analyses (e.g., PCR, Southern Blot, and cloning) microbiological applications (e.g., 
bacterial and fungal transformations), and bioinformatics. Other responsibilities include ordering laboratory supplies, monitoring and directing undergraduate students in the laboratory.

University of Maryland Baltimore County, Baltimore, MD

Laboratory Technician - 2003

Prepared materials and solutions for biochemistry laboratory sessions

University of Maryland Baltimore County, Baltimore, MD

Undergraduate Research Assistant - 2002 - 2003

Focused on incorporating a modified nucleotide into an RNA product corresponding to the third stem-loop of HIV-1 for analysis by mass spectrometry

\section{PUBLICATIONS}

\section{REFEREED JOURNAL ARTICLES}

Coyle, C. M., S. C. Kenaley, W. R. Rittenour, and D. G. Panaccione. 2007. Association of ergot alkaloids with conidiation in Aspergillus fumigatus. Mycologia 99(6):804-11.

Coyle, C. M. and D. G. Panaccione. 2005. An ergot alkaloid biosynthesis gene and clustered hypothetical genes from Aspergillus fumigatus. Appl. Environ. Microbiol. 71:3112-3118.

Panaccione, D. G. and C. M. Coyle. 2005. Abundant respirable ergot alkaloids from the common airborne fungus Aspergillus fumigatus. Appl. Environ. Microbiol. 71:3106-3111.

\section{BOOK CHAPTERS}

Panaccione, D. G., C. L.. Schardl, and C. M. Coyle. 2006. Pathways to diverse ergot alkaloid profiles in fungi, p. 23-52. In, J. T. Romeo (ed.), Recent Advances in Phytochemistry, vol. 40, Elsevier, Amsterdam.

\section{ABSTRACTS}

Coyle, C. M., K. E. Goetz, and D. G. Panaccione. 2009. Clustered genes common to Aspergillus fumigatus and ergot fungi control early steps in ergot alkaloid biosynthesis. Fungal Genet. Reports 56(Suppl):205. (Abstract)

Coyle, C. M., K. E. Goetz, and D. G. Panaccione. 2008. Clustered genes common to both Aspergillus fumigatus and ergot fungi control early steps within the ergot alkaloid pathway. Phytopathology 98:S214. (Abstract) 


\title{
PRESENTATIONS
}

Poster Presentation - Clustered genes common to both Aspergillus fumigatus and ergot fungi control early steps within the ergot alkaloid pathway

Fungal Genetics Conference, Asilomar, CA (2009)

Oral Presentation - Clustered genes common to both Aspergillus fumigatus and ergot fungi control early steps within the ergot alkaloid pathway

Davis College of Agriculture and Consumer Sciences, WVU, Morgantown, WV (2008)

American Phytopathological Society, Potomac Division, Morgantown, WV (2008)

Poster Session - Association of Ergot Alkaloids with Sporulation in Aspergillus fumigatus American Phytopathological Society, Potomac Division, Morgantown, WV (2008)

Sigma Xi, WVU, Morgantown, WV (2006)

Davis College of Agriculture and Consumer Sciences, WVU, Morgantown, WV (2006)

Poster Session - The Ergot Alkaloid Biosynthesis Gene Cluster from Aspergillus fumigatus Sigma Xi, WVU, Morgantown, WV (2005)

Davis College of Agriculture and Consumer Sciences, WVU, Morgantown, WV (2005)

\section{CONTRIBUTION TO OTHER PRESENTATIONS}

Panaccione, D.G., C.M. Coyle, E. Konnova, D.A. Potter, J.T. Stokes, J.R. Cipoletti, J.B.

Kotcon, K.E. Goetz, C. Machado, and C.L. Schardl. 2007. Toxin biosynthesis genes in ergot alkaloid-producing fungi (poster presentation).

USDA sponsored meeting "From Genes to Products," Bethesda, MD (2007)

American Phytopathological Society, Potomac Division, Morgantown, WV (2008)

\section{FACILITATION OF STUDENT RESEARCH/LABORATORY EXPERIENCE}

\author{
Graduate Students \\ Shaun Stanley - M. S. Agriculture \\ Kelly Mayhew - M. S. Applied Environmental Microbiology \\ Undergraduate Students \\ Dawn Chamberlain - Biochemistry \\ Michael Hamstead - Biology \\ Michael Chaisson - Biology \\ Kevin Junkins - Biology \\ Ashley Reynolds - Psychology \\ Brian Lewis - Biochemistry
}

\section{AWARDS}


Honorable Mention: Student Paper Presentation, American Phytopathology Society, Potomac

Division, Morgantown, WV (2008)

\section{SERVICE}

Local Arrangements Committee for American Phytopathology Society, Potomac Division Meeting (2008)

Division of Plant and Soil Science (Plant Pathology and Environmental Microbiology) Building

Safety Committee (2005-present)

\section{MEMBERSHIPS}

American Phytopathological Society, Potomac Division

Gamma Sigma Delta 